\title{
Enantioselective CuH-Catalyzed Reductive Coupling of Aryl Alkenes and Activated Carboxylic Acids
}

\author{
Jeffrey S. Bandar, Erhad Ascic, and Stephen L. Buchwald* \\ Department of Chemistry, Massachusetts Institute of Technology \\ Cambridge, Massachusetts 02139, United States
}

\section{Contents}

I. General Information S1

II. Optimization Studies for Table $1 \quad$ S2

III. Alcohol Synthesis: General Procedure and Substrate Scope S3

IV. Ketone Synthesis: General Procedure and Substrate Scope S12

V. Diastereoselective Reduction of Chiral Ketone 3a $\quad$ S14

VI. Preparation of Anhydride and Alkene Substrates $\quad$ S15

VII. References $\quad$ S16

VIII. HPLC Traces $\quad$ S17

IX. NMR Data S39

\section{General Information.}

General reagent information. All reactions were performed under a nitrogen atmosphere using the indicated method in the general procedures for optimization studies (II) and substrate scope sections (III-VI). Tetrahydrofuran (THF) was dried and deoxygenated by passage through two packed columns of neutral alumina and copper(II) oxide under a positive pressure of argon. 1,2-Bis $((2 S, 5 S) 2,5$-diphenylphospholano)ethane and 1,2-Bis $((2 R, 5 R) 2,5-$ diphenylphospholano)ethane (Ph-BPE) ligands were purchased from Strem Chemicals Inc. and Sigma Aldrich Co. and stored in a nitrogen filled glove box. Dimethoxy(methyl)silane (DMMS) was purchased from Tokyo Chemical Industry Co. (TCI) and stored in a nitrogen filled glove box at $-20{ }^{\circ} \mathrm{C}$ for long term storage. On a biweekly basis, DMMS was transferred to a vial that was stored under nitrogen in a freezer at $-5{ }^{\circ} \mathrm{C}$ for reaction set-up outside of the glove box. All other solvents and commercial reagents were used as received from Sigma Aldrich, Alfa Aesar, Acros Organics, TCI and Combi-Blocks, unless otherwise noted. Flash column chromatography was performed using 40-63 $\mu \mathrm{m}$ silica gel (SiliaFlash ${ }^{\circledR}$ F60 from Silicycle). Organic solutions were concentrated using a Buchi rotary evaporator.

General analytical information. All new compounds were characterized by NMR spectroscopy, IR spectroscopy, elemental analysis and melting point analysis (if solids). ${ }^{1} \mathrm{H},{ }^{13} \mathrm{C}$ and ${ }^{19} \mathrm{~F}$ NMR spectra were recorded in $\mathrm{CDCl}_{3}$ on a Bruker AMX-400 spectrometer. Data for ${ }^{1} \mathrm{H}$ NMR are reported as follows: chemical shift in reference to residual $\mathrm{CHCl}_{3}$ at $7.26 \mathrm{ppm}(\delta \mathrm{ppm})$, multiplicity $(\mathrm{s}=$ singlet, $\mathrm{br} \mathrm{s}=$ broad singlet, $\mathrm{d}=$ 
doublet, $\mathrm{t}=$ triplet, $\mathrm{q}=$ quartet, $\mathrm{dd}=$ doublet of doublets, $\mathrm{td}=$ triplet of doublets, $\mathrm{m}=$ multiplet), coupling constant $(\mathrm{Hz})$, and integration. Data for ${ }^{13} \mathrm{C}$ NMR are reported in terms of chemical shift in reference to the $\mathrm{CDCl}_{3}$ solvent signal $(77.16 \mathrm{ppm})$. Data for

${ }^{19} \mathrm{~F}-\mathrm{NMR}$ are reported in terms of chemical shift in reference to an external standard $(\alpha, \alpha, \alpha$-trifluorotoluene set to $\delta-63.7 \mathrm{ppm})$. IR spectra were recorded on a Thermo Scientific Nicolet iS5 spectrometer (iD5 ATR, diamond) and are reported in terms of frequency of absorption $\left(\mathrm{cm}^{-1}\right)$. Melting points were measured on a Mel-Temp capillary melting point apparatus. Optical rotations were measured using a Jasco P-1010 digital polarimeter. Elemental analyses were performed by Atlantic Microlabs Inc., Norcross, GA. ESI- and DART-MS spectrometric data were recorded on a Bruker Daltonics APEXIV 4.7 Tesla Fourier transform ion cyclotron resonance mass spectrometer (FT-ICR-MS). HPLC analysis was performed on an Agilent Technologies 1200 series instrument with Daicel Chiralpak and Chiralcel chiral columns $(25 \mathrm{~cm})$ using the given conditions. Thin-layer chromatography (TLC) was performed on silica gel $60 \AA$ $\mathrm{F}_{254}$ plates (SiliaPlate from Silicycle) and visualized with UV light or potassium permanganate stain. Preparatory thin-layer chromatography (Prep-TLC) was performed on silica gel GF with UV $254(20$ × $20 \mathrm{~cm}, 1000$ microns, catalog \# 02013 from Analtech) and visualized with UV light.

\section{Optimization Studies for Table 1}

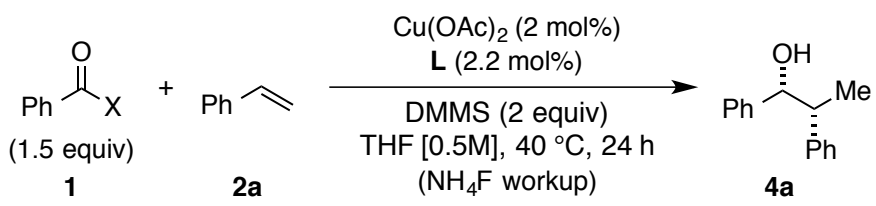

General procedure. In a nitrogen filled glove box, $\mathrm{Cu}(\mathrm{OAc})_{2}(1.8 \mathrm{mg}, 0.010 \mathrm{mmol}, 0.02$ equiv), phosphine ligand ( $0.011 \mathrm{mmol}, 0.022$ equiv) and THF ( $1 \mathrm{~mL})$ were added to an oven-dried reaction tube (Fisherbrand, 13 x $100 \mathrm{~mm}$, catalog no. 1495925A) containing a magnetic stir bar. The mixture was stirred for 10 min until a homogenous blue solution formed, at which time styrene $(57 \mu \mathrm{L}, 0.50 \mathrm{mmol}, 1.0$ equiv) and a benzoyl electrophile (0.75 mmol, 1.5 equiv) was added. After an additional $5 \mathrm{~min}$ of stirring, dimethoxymethylsilane $(247 \mu \mathrm{L}, 2.0 \mathrm{mmol}, 4.0$ equiv) was added. The reaction solution typically turned a yellow, orange or red color at this time. The reaction tube was capped (Thermo Scientific $13 \mathrm{~mm}$ screw cap with TEF/SIL septa, catalog no. C4015-66A), removed from the glove box, and inserted into an oil bath preheated to $40{ }^{\circ} \mathrm{C}$. The reaction solution was stirred for $24 \mathrm{~h}$, then allowed to cool to $\mathrm{rt}$, at which time sat. aqueous $\mathrm{NaHCO}_{3}(3 \mathrm{~mL})$ was added to quench the reaction and the mixture was extracted with EtOAc $(3 \times 2 \mathrm{~mL})$. The combined extracted organic solution was dried with anhydrous $\mathrm{Na}_{2} \mathrm{SO}_{4}$, filtered and concentrated in vacuo. A stock solution $(1 \mathrm{~mL})$ of 1,1,2,2-tetrachloroethane in $\mathrm{CDCl}_{3}(0.50 \mathrm{mmol}, 1.0$ equiv per $\mathrm{mL}$ of solution $)$ was added to dissolve the crude residue. ${ }^{1} \mathrm{H}$ NMR analysis of the solution was used to determine the product yield by comparison to 1,1,2,2-tetrachloroethane standard. The diastereomeric ratio was determined by integration of the methyl groups of the product at $1.38 \mathrm{ppm}$ (minor) and 1.11 ppm (major). 
For HPLC analysis, saturated $\mathrm{NH}_{4} \mathrm{~F}$ in methanol was added to the crude reaction residue and the mixture was stirred for $30 \mathrm{~min}$. The mixture was concentrated in vacuo, and then partitioned between sat. aqueous $\mathrm{NaHCO}_{3}(5 \mathrm{~mL})$ and EtOAc $(5 \mathrm{~mL})$. The EtOAc layer was removed, dried with anhydrous $\mathrm{Na}_{2} \mathrm{SO}_{4}$, filtered and concentrated in vacuo. The crude material was purified by silica gel column chromatography to yield the desired product as a white solid (10\% $\mathrm{Et}_{2} \mathrm{O}$ in hexanes). HPLC analysis: Chiralpak $\mathrm{AD}-\mathrm{H}$ $\left(\mathrm{Hex} / \mathrm{IPA}=95 / 5,1 \mathrm{~mL} / \mathrm{min}, 210 \mathrm{~nm}, 23^{\circ} \mathrm{C}\right.$ ), $12.8 \mathrm{~min}$ (minor), $13.8 \mathrm{~min}$ (major).

\section{Benzoyl electrophiles examined with (S)-DTBM-SEGPHOS (L1):}

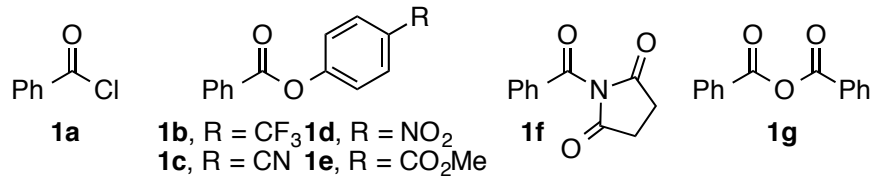

\begin{tabular}{|c|c|c|c|}
\hline Electrophile & Amount used & Product yield & Selectivity \\
\hline 1a & $105 \mathrm{mg}$ & $0 \%$ & - \\
\hline 1b & $200 \mathrm{mg}$ & $0 \%$ & - \\
\hline 1c & $167 \mathrm{mg}$ & $0 \%$ & - \\
\hline 1d & $182 \mathrm{mg}$ & $0 \%$ & - \\
\hline 1e & $192 \mathrm{mg}$ & $0 \%$ & - \\
\hline 1f & $152 \mathrm{mg}$ & $0 \%$ & - \\
\hline 1g & $170 \mathrm{mg}$ & $25 \%$ & $>20: 1 \mathrm{dr}, 95 \%$ ee \\
\hline
\end{tabular}

Phosphine ligands examined with $\mathrm{Bz}_{2} \mathrm{O}(1 \mathrm{~g})$ :

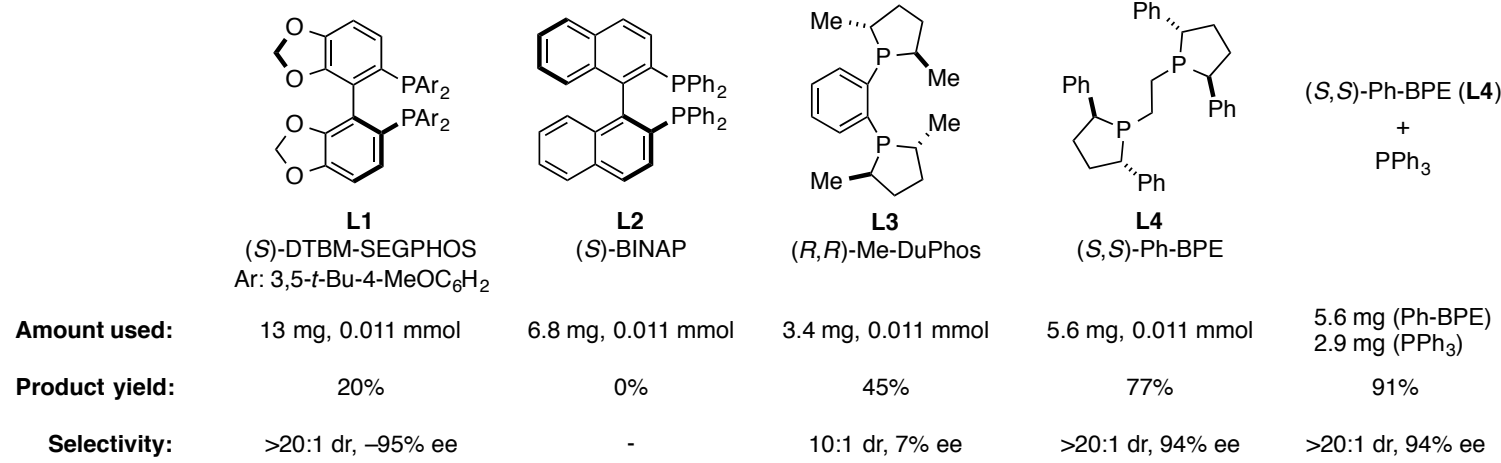

\section{Alcohol Synthesis: General Procedure and Substrate Scope}

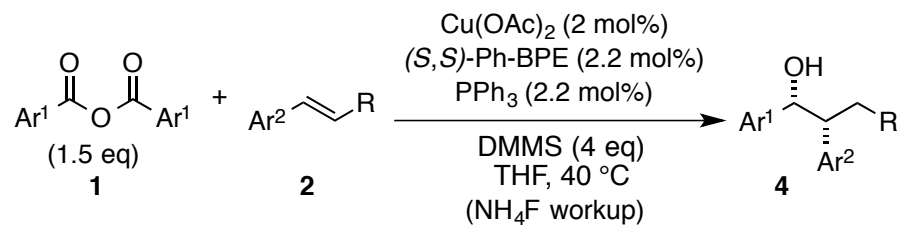


General procedure. An oven dried reaction tube (Fisherbrand, 16 x $125 \mathrm{~mm}$, catalog no. 1495925C) containing a magnetic stir bar was charged with alkene (if a solid, $1.0 \mathrm{mmol}$, 1.0 equiv) and aryl carboxylic acid anhydride (1.5 mmol, 1.5 equiv). The reaction tube was loosely capped (Cap: Kimble Chase Open Top S/T Closure catalog no. 7380415425; Septum: Thermo Scientific $2.6 \mathrm{~mm}$ silicone/PTFE catalog no. B7995-15), then brought into a nitrogen filled glove box. $\mathrm{Cu}(\mathrm{OAc})_{2}(3.6 \mathrm{mg}, 0.02 \mathrm{mmol}, 0.02$ equiv), $(S, S)$-Ph-BPE (11.1 mg, $0.022 \mathrm{mmol}, 0.022$ equiv) and $\mathrm{PPh}_{3}(5.8 \mathrm{mg}, 0.022 \mathrm{mmol}, 0.022$ equiv) was then added to the reaction tube. The reaction tube was capped, removed from the glove box, and put under a positive pressure of nitrogen via a needle line connected to a Schlenk line. THF ( $2 \mathrm{~mL})$ was added to the reaction tube via a syringe under nitrogen. The resulting mixture was stirred at $\mathrm{rt}$ for $10 \mathrm{~min}$ until a deep blue homogenous solution formed. If the alkene is a liquid, it was added via microsyringe at this time (1.0 mmol, 1.0 equiv). DMMS (493 $\mu \mathrm{L}, 4.0 \mathrm{mmol}, 4.0$ equiv) was then added via a syringe under nitrogen and the resulting solution was stirred at $\mathrm{rt}$ for an additional $5 \mathrm{~min}$ as the solution turned to a yellow, orange or red color. The nitrogen needle line was removed, the cap wrapped in parafilm, and the reaction tube inserted into an oil bath preheated to $40{ }^{\circ} \mathrm{C}$. After stirring for the time indicated for each reaction, the reaction tube was removed from the oil bath, cooled to $\mathrm{rt}$ and slowly quenched with sat. aqueous $\mathrm{NaHCO}_{3}(5 \mathrm{~mL}$, Caution: gas evolution observed). The mixture was extracted with EtOAc ( $3 \times 5 \mathrm{~mL})$ and the organic extracts were concentrated in vacuo. At this time, ${ }^{1} \mathrm{H}$ NMR analysis of the crude material was used to determine the diastereomeric ratio. The concentrated crude material was stirred with sat. $\mathrm{NH}_{4} \mathrm{~F}$ in $\mathrm{MeOH}(6 \mathrm{~mL})$ for $30 \mathrm{~min}$ and then reconcentrated to a crude solid. The crude material was partitioned between sat. aqueous $\mathrm{NaHCO}_{3}(15$ $\mathrm{mL})$ and EtOAc $(10 \mathrm{~mL})$. The EtOAc layer was separated, and the aqueous layer was extracted with EtOAc $(2 \times 10 \mathrm{~mL})$. The combined EtOAc solution was dried with anhydrous $\mathrm{Na}_{2} \mathrm{SO}_{4}$, filtered, concentrated in vacuo and the crude material was purified by silica gel column chromatography $\left(\mathrm{Et}_{2} \mathrm{O}\right.$ in hexanes).

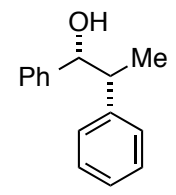

(1R,2R)-1,2-diphenylpropan-1-ol (4a). The general procedure was followed using styrene (114 $\mu \mathrm{L}, 1.0 \mathrm{mmol}, 1.0$ equiv) and benzoic anhydride (339 mg, $1.5 \mathrm{mmol}, 1.5$ equiv) with a $20 \mathrm{~h}$ reaction time. ${ }^{1} \mathrm{H}$ NMR analysis of the crude reaction material indicated $>20: 1 \mathrm{dr}$. Silica gel column chromatography ( 0 to $10 \% \mathrm{Et}_{2} \mathrm{O}$ in hexanes) yielded the title product as a white solid (Run 1: 195 mg, 0.919 mmol, 92\% yield; Run 2: $179 \mathrm{mg}, 0.843 \mathrm{mmol}, 84 \%$ yield). ${ }^{1} \mathbf{H}$ NMR (400 $\left.\mathrm{MHz}, \mathrm{CDCl}_{3}\right) \delta 7.52-7.21(\mathrm{~m}, 10 \mathrm{H}), 4.69(\mathrm{dd}, \mathrm{J}=8.7,2.3 \mathrm{~Hz}, 1 \mathrm{H}), 3.15-2.97(\mathrm{~m}, 1 \mathrm{H})$, $1.91(\mathrm{~d}, \mathrm{~J}=2.4 \mathrm{~Hz}, 1 \mathrm{H}), 1.12(\mathrm{~d}, \mathrm{~J}=7.0 \mathrm{~Hz}, 3 \mathrm{H}) .{ }^{13} \mathbf{C} \mathbf{N M R}\left(101 \mathrm{MHz}, \mathrm{CDCl}_{3}\right) \delta 163.7$, 161.3, 143.2, 138.4, 138.3, 128.9, 128.7, 128.6, 128.2, 127.2, 115.4, 115.1, 79.1, 48.4, 18.3. HRMS (DART) m/z calcd. for $\mathrm{C}_{15} \mathrm{H}_{20} \mathrm{NO}^{+}\left[\mathrm{M}+\mathrm{NH}_{4}\right]^{+}: 230.1539 ; 230.1530$ found. m.p. $68-70{ }^{\circ} \mathrm{C}$. IR (thin film) $3415,2954,1495,1451,1020,752,696 \mathrm{~cm}^{-1}$. Specific rotation $[\alpha]_{\mathrm{D}}{ }^{24}=+61.3\left(\mathrm{c}=1.0, \mathrm{CHCl}_{3}\right)$. HPLC analysis: Chiralpak AD-H (Hex/IPA = 95/5, $1.0 \mathrm{~mL} / \mathrm{min}, 210 \mathrm{~nm}, 23^{\circ} \mathrm{C}$ ), $12.9 \mathrm{~min}$ (minor), $13.8 \mathrm{~min}$ (major), 95\% ee. The absolute stereochemistry was determined by comparison of optical rotation measurement and HPLC retention times with literature data. ${ }^{1}$ The relative stereochemistry was determined by comparison of ${ }^{1} \mathrm{H}$ NMR data to reported values. ${ }^{2}$ The stereochemistry of all other alcohol products were assigned by analogy. 


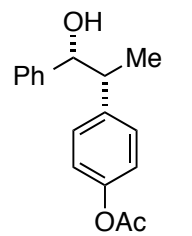

4-((1R,2R)-1-hydroxy-1-phenylpropan-2-yl)phenyl acetate (4b). The general procedure was followed using 4-acetoxystyrene $(153 \mu \mathrm{L}, 1.0 \mathrm{mmol}$, 1.0 equiv) and benzoic anhydride (339 mg, $1.5 \mathrm{mmol}, 1.5$ equiv) with a $12 \mathrm{~h}$ reaction time. ${ }^{1} \mathrm{H}$ NMR analysis of the crude reaction material indicated $>20: 1 \mathrm{dr}$. Silica gel column chromatography $\left(10 \% \mathrm{Et}_{2} \mathrm{O}\right.$ in hexanes) yielded the title product as a white solid (Run 1: $190 \mathrm{mg}, 0.703 \mathrm{mmol}, 70 \%$ yield; Run 2: $208 \mathrm{mg}$, $0.769 \mathrm{mmol}, 77 \%$ yield). ${ }^{1} \mathbf{H}$ NMR $\left(400 \mathrm{MHz}, \mathrm{CDCl}_{3}\right) \delta 7.43-7.24(\mathrm{~m}, 7 \mathrm{H}), 7.10(\mathrm{~d}, \mathrm{~J}=$ $8.5 \mathrm{~Hz}, 2 \mathrm{H}), 4.67$ (d, J = 8.5 Hz, 1H), $3.14-2.97$ (m, 1H), 2.33 (s, 3H), 1.88 (s, 1H), 1.10 $(\mathrm{d}, \mathrm{J}=7.1 \mathrm{~Hz}, 3 \mathrm{H}) .{ }^{13} \mathbf{C}$ NMR $\left(101 \mathrm{MHz}, \mathrm{CDCl}_{3}\right) \delta 169.7,149.6,142.6,141.0,129.2$, $128.5,128.0,127.1,121.7,79.7,47.7,21.3,18.5$. m.p. $70-72{ }^{\circ} \mathrm{C}$. IR (thin film) 3364 , 2962, 1756, 1504, 1213, 1199, 752, $698 \mathrm{~cm}^{-1}$. EA Calcd. for $\mathrm{C}_{17} \mathrm{H}_{18} \mathrm{O}_{3}: \mathrm{C}, 75.53 ; \mathrm{H}, 6.71$. Found: C, 75.39; H, 6.68. Specific rotation $[\alpha]_{\mathrm{D}}{ }^{24}=+59.3\left(\mathrm{c}=1.0, \mathrm{CHCl}_{3}\right)$. HPLC analysis: Chiralpak AD-H (Hex/IPA =97/3, $\left.1.0 \mathrm{~mL} / \mathrm{min}, 230 \mathrm{~nm}, 23^{\circ} \mathrm{C}\right), 36.0 \mathrm{~min}$ (major), $38.6 \mathrm{~min}$ (minor), $98 \%$ ee.

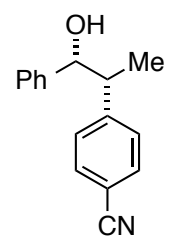

4-((1R,2R)-1-hydroxy-1-phenylpropan-2-yl)benzonitrile (4c). The general procedure was followed using 4-cyanostyrene (133 $\mathrm{mg}, 1.0 \mathrm{mmol}, 1.0$ equiv) and benzoic anhydride (339 $\mathrm{mg}, 1.5 \mathrm{mmol}, 1.5$ equiv) with a $24 \mathrm{~h}$ reaction time. ${ }^{1} \mathrm{H}$ NMR analysis of the crude reaction material indicated 3:1 dr. Silica gel column chromatography ( 5 to $20 \% \mathrm{Et}_{2} \mathrm{O}$ in hexanes) yielded the title product as a white solid (Run 1: $220 \mathrm{mg}, 0.927 \mathrm{mmol}, 93 \%$ yield; Run 2: 194 mg, 0.819 mmol, 82\% yield). ${ }^{1} \mathbf{H}$ NMR (400 $\left.\mathrm{MHz}, \mathrm{CDCl}_{3}\right)$ Major diastereomer: $\delta 7.63$ $(\mathrm{d}, \mathrm{J}=8.2 \mathrm{~Hz}, 2 \mathrm{H}), 7.44-7.27(\mathrm{~m}, 7 \mathrm{H}), 4.78-4.71(\mathrm{~m}, 1 \mathrm{H}), 3.17-3.08(\mathrm{~m}, 1 \mathrm{H}), 1.91$ $(\mathrm{d}, \mathrm{J}=2.7 \mathrm{~Hz}, 1 \mathrm{H}), 1.16(\mathrm{~d}, \mathrm{~J}=7.1 \mathrm{~Hz}, 3 \mathrm{H})$; Minor diastereomer: $\delta 7.52(\mathrm{~d}, \mathrm{~J}=8.3 \mathrm{~Hz}$, 2H), $7.30-7.21(\mathrm{~m}, 5 \mathrm{H}), 7.19-7.12(\mathrm{~m}, 2 \mathrm{H}), 4.82-4.78(\mathrm{~m}, 1 \mathrm{H}), 3.19(\mathrm{~m}, 1 \mathrm{H}), 2.02$ $(\mathrm{d}, \mathrm{J}=3.3 \mathrm{~Hz}, 1 \mathrm{H}), 1.39(\mathrm{~d}, \mathrm{~J}=7.0 \mathrm{~Hz}, 3 \mathrm{H}) .{ }^{13} \mathbf{C} \mathbf{N M R}\left(101 \mathrm{MHz}, \mathrm{CDCl}_{3}\right) \delta 149.5$, *149.5, 142.4, *142.4, 132.2, 131.9, 129.1, 128.6, 128.5, 128.2, 128.2, 127.8, 126.7, $126.4,119.1,110.4, * 110.2,79.1, * 78.5,47.9, * 47.6,18.2, * 15.6$, *indicates minor diastereomer. HRMS (DART) $\mathrm{m} / \mathrm{z}$ calcd. for $\mathrm{C}_{16} \mathrm{H}_{14} \mathrm{NO}^{-}[\mathrm{M}-\mathrm{H}]^{-}: 236.1081 ; 236.1034$ found. m.p. $83-85{ }^{\circ} \mathrm{C}$. IR (thin film) 3543, 3460, 2967, 2225, 1604, 1451, 852, 765, 699 $\mathrm{cm}^{-1}$. Specific rotation $[\alpha]_{\mathrm{D}}{ }^{24}=+9.8\left(\mathrm{c}=1.0, \mathrm{CHCl}_{3}\right)$. HPLC analysis: Chiralcel OD-H $\left(\mathrm{Hex} / \mathrm{IPA}=95 / 5,0.8 \mathrm{~mL} / \mathrm{min}, 230 \mathrm{~nm}, 23^{\circ} \mathrm{C}\right.$ ), 31.8 (minor of minor diastereomer), 38.0 min (minor of major diastereomer), $39.7 \mathrm{~min}$ (major of minor diastereomer), $47.5 \mathrm{~min}$ (major of major diastereomer), 37\% ee for major diastereomer and $92 \%$ ee for minor diastereomer.

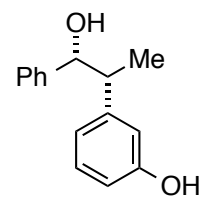

3-((1R,2R)-1-hydroxy-1-phenylpropan-2-yl)phenol (4d). For this substrate, 5 equiv of DMMS $(616 \mu \mathrm{L}, 5.0 \mathrm{mmol})$ was used. The general procedure was followed using 3-vinylphenol (120 mg, $1.0 \mathrm{mmol}, 1.0$ equiv) and benzoic anhydride (339 $\mathrm{mg}, 1.5 \mathrm{mmol}, 1.5$ equiv) with a $7 \mathrm{~h}$ reaction time. ${ }^{1} \mathrm{H}$ NMR analysis of the crude reaction material indicated $>20: 1 \mathrm{dr}$. Silica gel column chromatography (10 to $20 \% \mathrm{Et}_{2} \mathrm{O}$ in hexanes) yielded the title product 
as a white solid (Run 1: $152 \mathrm{mg}, 0.666 \mathrm{mmol}, 67 \%$ yield; Run 2: $148 \mathrm{mg}, 0.648 \mathrm{mmol}$, $65 \%$ yield). ${ }^{1} \mathbf{H}$ NMR $\left(400 \mathrm{MHz}, \mathrm{CDCl}_{3}\right) \delta 7.52-7.13(\mathrm{~m}, 6 \mathrm{H}), 6.91(\mathrm{dd}, \mathrm{J}=7.6,1.3 \mathrm{~Hz}$, $1 \mathrm{H}), 6.84-6.71(\mathrm{~m}, 2 \mathrm{H}), 4.91(\mathrm{~s}, 1 \mathrm{H}), 4.66(\mathrm{~d}, \mathrm{~J}=8.8 \mathrm{~Hz}, 1 \mathrm{H}), 2.99(\mathrm{dq}, \mathrm{J}=8.6,7.1 \mathrm{~Hz}$, $1 \mathrm{H}), 1.95(\mathrm{~s}, 1 \mathrm{H}), 1.07(\mathrm{dd}, \mathrm{J}=7.1,0.9 \mathrm{~Hz}, 3 \mathrm{H}) .{ }^{13} \mathbf{C} \mathbf{N M R}\left(101 \mathrm{MHz}, \mathrm{CDCl}_{3}\right) \delta 156.0$, $145.5,142.4,130.1,128.5,128.1,127.1,120.5,115.1,114.1,79.9,48.2,18.5$. HRMS (DART) m/z calcd. for $\mathrm{C}_{15} \mathrm{H}_{15} \mathrm{O}_{2}^{-}[\mathrm{M}-\mathrm{H}]^{-}: 227.1078 ; 227.1063$ found. m.p. 103-105 ${ }^{\circ} \mathrm{C}$. IR (thin film) 3248, 1600, 1451, 1204, 1005, 926, 791, $702 \mathrm{~cm}^{-1}$. Specific rotation $[\alpha]_{\mathrm{D}}{ }^{24}=+56.4\left(\mathrm{c}=1.0, \mathrm{CHCl}_{3}\right)$. HPLC analysis: Chiralcel OD-H $(\mathrm{Hex} / \mathrm{IPA}=90 / 10,1.0$ $\mathrm{mL} / \mathrm{min}, 230 \mathrm{~nm}, 23^{\circ} \mathrm{C}$ ), $33.8 \mathrm{~min}$ (minor), $36.0 \mathrm{~min}$ (major), $95 \%$ ee.

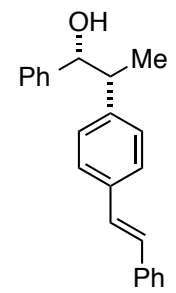

$(1 R, 2 R)-1-p h e n y l-2-(4-((E)-s t y r y l) p h e n y l) p r o p a n-1-o l \quad(4 e) . \quad$ For this substrate, the reaction was run at $\mathrm{rt}$. The general procedure was followed using (E)-1-styryl-4-vinylbenzene (206 mg, $1.0 \mathrm{mmol}, 1.0$ equiv) and benzoic anhydride (339 mg, $1.5 \mathrm{mmol}, 1.5$ equiv) with a $36 \mathrm{~h}$ reaction time. ${ }^{1} \mathrm{H}$ NMR analysis of the crude reaction material indicated 15:1 dr. Silica gel column chromatography ( 0 to $10 \% \mathrm{Et}_{2} \mathrm{O}$ in hexanes) yielded the title product as a white solid (Run 1: $248 \mathrm{mg}, 0.789 \mathrm{mmol}, 79 \%$ yield; Run 2: $245 \mathrm{mg}, 0.779$ mmol, 78\% yield). ${ }^{1} \mathbf{H}$ NMR $\left(400 \mathrm{MHz}, \mathrm{CDCl}_{3}\right) \delta 7.68-7.50(\mathrm{~m}, 4 \mathrm{H}), 7.50-7.24(\mathrm{~m}$, $10 \mathrm{H}), 7.16(\mathrm{~s}, 2 \mathrm{H}), 4.71(\mathrm{dd}, \mathrm{J}=8.6,2.4 \mathrm{~Hz}, 1 \mathrm{H}), 3.17-3.01(\mathrm{~m}, 1 \mathrm{H}), 1.92(\mathrm{~d}, \mathrm{~J}=2.5$ $\mathrm{Hz}, 1 \mathrm{H}), 1.14(\mathrm{~d}, \mathrm{~J}=7.0 \mathrm{~Hz}, 3 \mathrm{H}) .{ }^{13} \mathbf{C} \mathbf{N M R}\left(101 \mathrm{MHz}, \mathrm{CDCl}_{3}\right) \delta 143.1,142.7,137.5$, $136.2,128.8,128.6,128.5,128.5,128.4,128.0,127.7,127.1,126.9,126.6,79.8,48.0$, 18.4. m.p. $149-151{ }^{\circ} \mathrm{C}$. IR (thin film) 3372, 3026, 2961, 1451, 962, 812, 746, $689 \mathrm{~cm}^{-1}$. EA Calcd. for $\mathrm{C}_{23} \mathrm{H}_{22} \mathrm{O}$ : C, 87.86; H, 7.05. Found: C, 87.63; H, 7.22. Specific rotation $[\alpha]_{\mathrm{D}}^{24}=+58.8\left(\mathrm{c}=1.0, \mathrm{CHCl}_{3}\right)$. HPLC analysis: Chiralcel OD-H $(\mathrm{Hex} / \mathrm{IPA}=95 / 5,1.0$ $\mathrm{mL} / \mathrm{min}, 210 \mathrm{~nm}, 23^{\circ} \mathrm{C}$ ), $19.5 \mathrm{~min}$ (minor), $27.2 \mathrm{~min}$ (major), $92 \%$ ee.

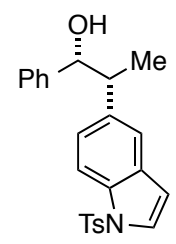

(1R,2R)-1-phenyl-2-(1-tosyl-1H-indol-5-yl)propan-1-ol (4f). For this substrate, $4 \mathrm{~mol} \%$ catalyst consisting of $\mathrm{Cu}(\mathrm{OAc})_{2}$ (7.2 mg, $0.04 \mathrm{mmol}, 0.04$ equiv), $(S, S)$-Ph-BPE (22.2 mg, 0.044 mmol, 0.044 equiv) and $\mathrm{PPh}_{3}(11.5$ $\mathrm{mg}, 0.044 \mathrm{mmol}, 0.044$ equiv) was used. The general procedure was followed using 1-tosyl-5-vinyl-1 $H$-indole (297 mg, $1.0 \mathrm{mmol}, 1.0$ equiv) and benzoic anhydride (339 mg, $1.5 \mathrm{mmol}, 1.5$ equiv) with a $24 \mathrm{~h}$ reaction time. ${ }^{1} \mathrm{H}$ NMR analysis of the crude reaction material indicated $>20: 1$ dr. Silica gel column chromatography $\left(1 \% \mathrm{MeOH}\right.$ in $\left.\mathrm{CH}_{2} \mathrm{Cl}_{2}\right)$ yielded the title product as a white solid (Run 1: $301 \mathrm{mg}, 0.743 \mathrm{mmol}, 74 \%$ yield; Run 2: $274 \mathrm{mg}, 0.676 \mathrm{mmol}, 68 \%$ yield). ${ }^{1}$ H NMR (400 $\left.\mathrm{MHz}, \mathrm{CDCl}_{3}\right) \delta 7.98(\mathrm{~d}, \mathrm{~J}=8.6 \mathrm{~Hz}, 1 \mathrm{H}), 7.81(\mathrm{~d}, \mathrm{~J}=8.4 \mathrm{~Hz}, 2 \mathrm{H}), 7.59(\mathrm{~d}, \mathrm{~J}=3.6 \mathrm{~Hz}$, 1H), $7.49(\mathrm{~d}, \mathrm{~J}=1.6 \mathrm{~Hz}, 1 \mathrm{H}), 7.44-7.20(\mathrm{~m}, 8 \mathrm{H}), 6.66(\mathrm{dd}, \mathrm{J}=3.6,0.8 \mathrm{~Hz}, 1 \mathrm{H}), 4.67$ $(\mathrm{dd}, \mathrm{J}=8.8,2.1 \mathrm{~Hz}, 1 \mathrm{H}), 3.09(\mathrm{dd}, \mathrm{J}=8.8,7.0 \mathrm{~Hz}, 1 \mathrm{H}), 2.37(\mathrm{~s}, 3 \mathrm{H}), 1.82$ (d, J = 2.3 Hz, 1H), $1.10(\mathrm{~d}, \mathrm{~J}=7.0 \mathrm{~Hz}, 3 \mathrm{H}) .{ }^{13} \mathbf{C}$ NMR $\left(101 \mathrm{MHz}, \mathrm{CDCl}_{3}\right) \delta 145.1,142.7,138.6,135.5$, $134.0,131.3,130.0,128.5,128.0,127.1,127.0,126.8,124.8,120.7,113.8,109.0,80.0$, 48.2, 21.7, 18.9. m.p. $60-62{ }^{\circ} \mathrm{C}$. IR (thin film) 3546, 2968, 1368, 1269, 1170, 1127, 673 $\mathrm{cm}^{-1}$. EA Calcd. for $\mathrm{C}_{24} \mathrm{H}_{22} \mathrm{NO}_{3} \mathrm{~S}$ : C, 71.09; H, 5.72. Found: C, 70.79; H, 5.64. Specific rotation $[\alpha]_{\mathrm{D}}{ }^{24}=+28.7\left(\mathrm{c}=1.0, \mathrm{CHCl}_{3}\right)$. HPLC analysis: Chiralpak AD-H $(\mathrm{Hex} / \mathrm{IPA}=$ $80 / 20,1.0 \mathrm{~mL} / \mathrm{min}, 210 \mathrm{~nm}, 23^{\circ} \mathrm{C}$ ), $21.9 \mathrm{~min}$ (minor), $24.8 \mathrm{~min}$ (major), 99\% ee. 


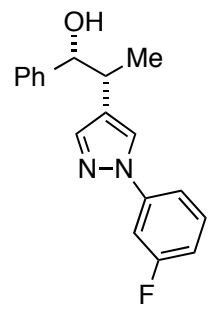

(1R,2R)-2-(1-(3-fluorophenyl)-1H-pyrazol-4-yl)-1-phenylpropan-1-ol (4g). A modified procedure was used for this substrate. An oven dried reaction tube (Fisherbrand, $16 \times 125 \mathrm{~mm}$, catalog no. 1495925C) containing a magnetic stir bar was charged with 1-(3-fluorophenyl)-4vinyl- $1 H$-pyrazole (188 mg, $1.0 \mathrm{mmol}, 1.0$ equiv). The reaction tube was loosely capped (Cap: Kimble Chase Open Top S/T Closure catalog no. 73804-15425; Septum: Thermo Scientific $2.6 \mathrm{~mm}$ silicone/PTFE catalog no. B7995-15), then brought into a nitrogen filled glove box. $\mathrm{Cu}(\mathrm{OAc})_{2}(7.2 \mathrm{mg}, 0.04$ mmol, 0.04 equiv), (S,S)-Ph-BPE (22.2 mg, 0.044 mmol, 0.044 equiv) and $\mathrm{PPh}_{3}(11.5$ $\mathrm{mg}, 0.044 \mathrm{mmol}, 0.044$ equiv) was then added to the reaction tube. The reaction tube was capped, removed from the glove box, and put under a positive pressure of nitrogen via a needle line connected to a Schlenk line. THF $(2 \mathrm{~mL})$ was added to the reaction tube via a syringe under nitrogen. The resulting mixture was stirred at $\mathrm{rt}$ for 10 min until a deep blue homogenous solution formed. DMMS ( $493 \mu \mathrm{L}, 4.0 \mathrm{mmol}$, 4.0 equiv) was then added via a syringe under nitrogen and the resulting solution was stirred at $\mathrm{rt}$ for an additional $2 \mathrm{~min}$ as the solution turned to an orange color. The nitrogen needle line was removed, the cap wrapped in parafilm, and the reaction tube inserted into an oil bath preheated to $40{ }^{\circ} \mathrm{C}$. At this time, a syringe pump (Harvard Apparatus PHD 2000) was used to add a benzoic anhydride (452 $\mathrm{mg}, 2.00 \mathrm{mmol}, 2.0$ equiv) in THF ( $1 \mathrm{~mL})$ solution drop wise over $4 \mathrm{~h}(5.8 \mu \mathrm{L} / \mathrm{min}$ addition rate). After stirring for an additional $44 \mathrm{~h}$, the reaction tube was removed from the oil bath, cooled to $\mathrm{rt}$ and the reaction was slowly quenched with sat. aqueous $\mathrm{NaHCO}_{3}(5 \mathrm{~mL}$, Caution: gas evolution observed). The mixture was extracted with EtOAc $(3 \times 5 \mathrm{~mL})$ and the organic extracts were concentrated in vacuo. At this time, ${ }^{1} \mathrm{H}$ NMR analysis of the crude material was used to determine the yield (Run 1: 45\% NMR yield; Run 2: 44\% NMR yield) and indicated $>20: 1$ diastereomeric ratio $(1,1,2,2$, -tetrachlorethane added as internal standard, $52 \mu \mathrm{L}, 0.50$ mmol, 0.50 equiv).

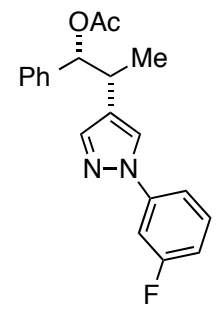

(1R,2R)-2-(1-(3-fluorophenyl)-1H-pyrazol-4-yl)-1-phenylpropyl

acetate. The concentrated crude material from above was stirred with sat. $\mathrm{NH}_{4} \mathrm{~F}$ in $\mathrm{MeOH}(6 \mathrm{~mL})$ for $30 \mathrm{~min}$ and then reconcentrated to a crude solid. The crude material was partitioned between sat. aqueous $\mathrm{NaHCO}_{3}$ $(15 \mathrm{~mL})$ and EtOAc $(10 \mathrm{~mL})$. The EtOAc layer was separated, and the aqueous layer was extracted with EtOAc $(2 \times 10 \mathrm{~mL})$. The combined EtOAc solution was dried with anhydrous $\mathrm{Na}_{2} \mathrm{SO}_{4}$, filtered, concentrated in vacuo and the crude material was purified by silica gel column chromatography $(20 \%$ $\mathrm{Et}_{2} \mathrm{O}$ in hexanes). The purified alcohol was unstable and could not be fully characterized. The purified alcohol was immediately dissolved in $\mathrm{Et}_{2} \mathrm{O}(5 \mathrm{~mL})$ and acetyl chloride (143 $\mu \mathrm{L}, 2.0 \mathrm{mmol})$ and triethylamine $(279 \mu \mathrm{L}, 2.0 \mathrm{mmol})$ were added. The reaction mixture was stirred for $30 \mathrm{~min}$, then quenched with $\mathrm{H}_{2} \mathrm{O}$ and extracted with $\mathrm{Et}_{2} \mathrm{O}$. The extracted $\mathrm{Et}_{2} \mathrm{O}$ solution was dried with anhydrous $\mathrm{Na}_{2} \mathrm{SO}_{4}$, filtered, concentrated in vacuo and the crude material purified by silica gel column chromatography $\left(10 \% \mathrm{Et}_{2} \mathrm{O}\right.$ in hexanes) to yield the title product as a colorless oil $\left(145 \mathrm{mg}, 0.429 \mathrm{mmol}, 43 \%\right.$ two-step yield). ${ }^{1} \mathbf{H}$ NMR $\left(400 \mathrm{MHz}, \mathrm{CDCl}_{3}\right) \delta 7.64(\mathrm{~s}, 1 \mathrm{H}), 7.57(\mathrm{~s}, 1 \mathrm{H}), 7.50-7.22(\mathrm{~m}, 8 \mathrm{H}), 7.06-6.90$ 
$(\mathrm{m}, 1 \mathrm{H}), 5.78(\mathrm{~d}, \mathrm{~J}=7.3 \mathrm{~Hz}, 1 \mathrm{H}), 3.29(\mathrm{~m}, 1 \mathrm{H}), 2.06(\mathrm{~d}, \mathrm{~J}=1.1 \mathrm{~Hz}, 3 \mathrm{H}), 1.22(\mathrm{dd}, \mathrm{J}=$ 7.1, $1.2 \mathrm{~Hz}, 3 \mathrm{H}) .{ }^{13} \mathrm{C}$ NMR $\left(101 \mathrm{MHz}, \mathrm{CDCl}_{3}\right) \delta 170.1,164.6,162.2,141.7,141.6$, $141.3,139.1,130.9,130.8,128.4,128.2,127.2$, 125.4, 124.9, 114.0, 114.0, 113.1, 112.9, 106.7, 106.4, 79.7, 35.9, 21.3, 17.8. ${ }^{19} \mathbf{F}$ NMR $\left(376 \mathrm{MHz} \mathrm{CDCl}_{3}\right) \delta-111.0$. IR (thin film) 2973, 1733, 1613, 1600, 1497, 1230, 864, $699 \mathrm{~cm}^{-1}$. EA Calcd. for $\mathrm{C}_{20} \mathrm{H}_{19} \mathrm{FN}_{2} \mathrm{O}_{2}$ : C, 70.99; H, 5.66. Found: $\mathrm{C}, 71.05 ; \mathrm{H}, 5.85$. Specific rotation $[\alpha]_{\mathrm{D}}{ }^{24}=+52.5(\mathrm{c}=0.5$, $\left.\mathrm{CHCl}_{3}\right)$. HPLC analysis: Chiralcel OD-H (Hex/IPA = 95/5, $\left.1.0 \mathrm{~mL} / \mathrm{min}, 210 \mathrm{~nm}, 23^{\circ} \mathrm{C}\right)$, 7.8 min (minor), 9.1 min (major), $98 \%$ ee.

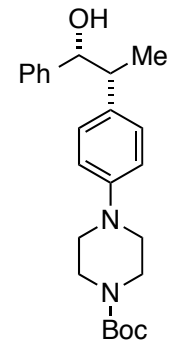

tert-butyl

4-(4-((1R,2R)-1-hydroxy-1-phenylpropan-2yl)phenyl)piperazine-1-carboxylate (4h). For this substrate, $4 \mathrm{~mol} \%$ catalyst consisting of $\mathrm{Cu}(\mathrm{OAc})_{2}(7.2 \mathrm{mg}, 0.04 \mathrm{mmol}, 0.04$ equiv), $(S, S)-\mathrm{Ph}-$ BPE (22.2 mg, $0.044 \mathrm{mmol}, 0.044$ equiv) and $\mathrm{PPh}_{3}(11.5 \mathrm{mg}, 0.044 \mathrm{mmol}$, 0.044 equiv) was used. The general procedure was followed using tert-butyl 4-(4-vinylphenyl)piperazine-1-carboxylate (288 $\mathrm{mg}, 1.0 \mathrm{mmol}, 1.0$ equiv) and benzoic anhydride (452 $\mathrm{mg}, 2.0 \mathrm{mmol}, 2.0$ equiv) with a $48 \mathrm{~h}$ reaction time. ${ }^{1} \mathrm{H}$ NMR analysis of the crude reaction material indicated $>20: 1 \mathrm{dr}$. Silica gel column chromatography (10 to $20 \% \mathrm{Et}_{2} \mathrm{O}$ in hexanes) yielded the title product as a white solid (Run 1: $222 \mathrm{mg}, 0.560 \mathrm{mmol}, 56 \%$ yield; Run 2: $212 \mathrm{mg}, 0.535 \mathrm{mmol}$, $54 \%$ yield). ${ }^{1} \mathbf{H}$ NMR (400 MHz, $\left.\mathrm{CDCl}_{3}\right) \delta 7.42-7.28(\mathrm{~m}, 5 \mathrm{H}), 7.25-7.20(\mathrm{~m}, 2 \mathrm{H})$, $6.95(\mathrm{~d}, \mathrm{~J}=8.6 \mathrm{~Hz}, 2 \mathrm{H}), 4.61(\mathrm{dd}, \mathrm{J}=8.7,2.1 \mathrm{~Hz}, 1 \mathrm{H}), 3.61(\mathrm{t}, \mathrm{J}=5.2 \mathrm{~Hz}, 4 \mathrm{H}), 3.15$ (t, J $=5.1 \mathrm{~Hz}, 4 \mathrm{H}), 2.97(\mathrm{dd}, \mathrm{J}=8.4,6.9 \mathrm{~Hz}, 1 \mathrm{H}), 1.92(\mathrm{~d}, \mathrm{~J}=2.3 \mathrm{~Hz}, 1 \mathrm{H}), 1.51(\mathrm{~s}, 9 \mathrm{H}), 1.07$ $(\mathrm{d}, \mathrm{J}=7.1 \mathrm{~Hz}, 3 \mathrm{H}) .{ }^{13} \mathbf{C} \mathbf{N M R}\left(101 \mathrm{MHz}, \mathrm{CDCl}_{3}\right) \delta 154.9,150.3,142.8,134.9,128.9$, 128.4, 127.8, 127.1, 117.0, 80.0, 79.9, 49.6, 47.5, 28.6, 18.5. m.p. 112-114 ${ }^{\circ} \mathrm{C}$. IR (thin film) 3337, 2969, 1684, 1413, 1221, 1123, 707, $689 \mathrm{~cm}^{-1}$. EA Calcd. for $\mathrm{C}_{24} \mathrm{H}_{32} \mathrm{~N}_{2} \mathrm{O}_{3}$ : C, 72.70; H, 8.13. Found: C, 72.71; H, 8.07. Specific rotation $[\alpha]_{\mathrm{D}}{ }^{24}=+46.2(\mathrm{c}=1.0$, $\left.\mathrm{CHCl}_{3}\right)$. HPLC analysis: Chiralpak AD-H (Hex/IPA $=90 / 10,1.0 \mathrm{~mL} / \mathrm{min}, 254 \mathrm{~nm}$, $23^{\circ} \mathrm{C}$ ), $19.9 \min$ (minor), $24.9 \mathrm{~min}$ (major), $98 \%$ ee.

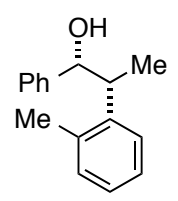

(1R,2R)-1-phenyl-2-(o-tolyl)propan-1-ol (4i). For this substrate, $4 \mathrm{~mol} \%$ catalyst consisting of $\mathrm{Cu}(\mathrm{OAc})_{2}(7.2 \mathrm{mg}, 0.04 \mathrm{mmol}, 0.04$ equiv), $(S, S)-\mathrm{Ph}-$ BPE (22.2 mg, $0.044 \mathrm{mmol}, 0.044$ equiv) and $\mathrm{PPh}_{3}(11.5 \mathrm{mg}, 0.044 \mathrm{mmol}$, 0.044 equiv) was used. The general procedure was followed using 2methylstyrene (130 $\mu \mathrm{L}, 1.0 \mathrm{mmol}, 1.0$ equiv) and benzoic anhydride (452 mg, 2.0 mmol, 2.0 equiv) with a $48 \mathrm{~h}$ reaction time. ${ }^{1} \mathrm{H}$ NMR analysis of the crude reaction material indicated $10: 1 \mathrm{dr}$. Silica gel column chromatography (5 to $10 \% \mathrm{Et}_{2} \mathrm{O}$ in hexanes) yielded the title product as a colorless oil (Run 1: $104 \mathrm{mg}, 0.460 \mathrm{mmol}, 46 \%$ yield; Run 2: $120 \mathrm{mg}, 0.530 \mathrm{mmol}, 53 \%$ yield). ${ }^{1} \mathbf{H}$ NMR (400 $\left.\mathrm{MHz}, \mathrm{CDCl}_{3}\right) \delta 7.52-$ $7.11(\mathrm{~m}, 9 \mathrm{H}), 4.82-4.69(\mathrm{~m}, 1 \mathrm{H}), 3.48-3.30(\mathrm{~m}, 1 \mathrm{H}), 2.41(\mathrm{~s}, 3 \mathrm{H}), 1.88(\mathrm{~d}, \mathrm{~J}=2.0 \mathrm{~Hz}$, $1 \mathrm{H}), 1.06(\mathrm{~d}, \mathrm{~J}=7.0 \mathrm{~Hz}, 3 \mathrm{H}) .{ }^{13} \mathbf{C}$ NMR $\left(101 \mathrm{MHz}, \mathrm{CDCl}_{3}\right) \delta 142.9,141.8,137.3,130.7$, $128.4,128.0,127.2,126.7,126.6,126.0,80.0,42.8,20.1,18.4$. IR (thin film) 3434, 2966, 1493, 1452, 1270, 766, $699 \mathrm{~cm}^{-1}$. EA Calcd. for $\mathrm{C}_{16} \mathrm{H}_{18} \mathrm{O}$ : C, 84.91; H, 8.02. Found: C, 84.65; H, 8.02. Specific rotation $[\alpha]_{\mathrm{D}}{ }^{24}=+38.5\left(\mathrm{c}=1.0, \mathrm{CHCl}_{3}\right)$. HPLC analysis: 
Chiralpak AD-H (Hex/IPA = 95/5, $\left.1.0 \mathrm{~mL} / \mathrm{min}, 230 \mathrm{~nm}, 23^{\circ} \mathrm{C}\right), 15.5 \mathrm{~min}$ (major), 17.2 min (minor), $86 \%$ ee.

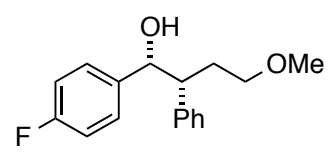

(1R,2R)-1-(4-fluorophenyl)-4-methoxy-2-phenylbutan-1-ol (4j). For this substrate, $4 \mathrm{~mol} \%$ catalyst consisting of $\mathrm{Cu}(\mathrm{OAc})_{2}(7.2 \mathrm{mg}$, $0.04 \mathrm{mmol}, 0.04$ equiv), $(S, S)$-Ph-BPE (22.2 mg, $0.044 \mathrm{mmol}, 0.044$ equiv) and $\mathrm{PPh}_{3}(11.5 \mathrm{mg}, 0.044 \mathrm{mmol}, 0.044$ equiv) was used. The general procedure was followed using cinnamyl methyl ether (148 $\mathrm{mg}, 1.0 \mathrm{mmol}, 1.0$ equiv) and 4-fluorobenzoic anhydride (524 mg, $2.0 \mathrm{mmol}, 2.0$ equiv) with a $36 \mathrm{~h}$ reaction time. ${ }^{1} \mathrm{H}$ NMR analysis of the crude reaction material indicated $>20: 1 \mathrm{dr}$. Silica gel column chromatography $\left(15 \% \mathrm{Et}_{2} \mathrm{O}\right.$ in hexanes) yielded the title product as a colorless oil (Run 1: $71 \mathrm{mg}, 0.258 \mathrm{mmol}, 26 \%$ yield; Run 2: $74 \mathrm{mg}, 0.269 \mathrm{mmol}, 27 \%$ yield). ${ }^{1} \mathbf{H}$ NMR $\left(400 \mathrm{MHz}, \mathrm{CDCl}_{3}\right) \delta 7.42-7.19(\mathrm{~m}, 7 \mathrm{H}), 7.02(\mathrm{t}, \mathrm{J}=8.7 \mathrm{~Hz}, 2 \mathrm{H}), 4.80(\mathrm{dd}, \mathrm{J}=$ 7.8, $2.3 \mathrm{~Hz}, 1 \mathrm{H}), 3.25-3.20(\mathrm{~m}, 1 \mathrm{H}), 3.18(\mathrm{~s}, 3 \mathrm{H}), 3.16(\mathrm{~m}, 1 \mathrm{H}), 3.12-2.94(\mathrm{~m}, 1 \mathrm{H})$, $2.12(\mathrm{~d}, \mathrm{~J}=2.8 \mathrm{~Hz}, 1 \mathrm{H}), 1.87-1.73(\mathrm{~m}, 2 \mathrm{H}) .{ }^{13} \mathbf{C}$ NMR $\left(101 \mathrm{MHz}, \mathrm{CDCl}_{3}\right) \delta 163.6$, $161.2,140.6,138.2,138.2,128.9,128.8,128.7,128.6,127.3,115.3,115.1,77.9,70.6$, 58.6, 51.1, 32.1. ${ }^{19}$ F NMR $\left(376 \mathrm{MHz}, \mathrm{CDCl}_{3}\right) \delta-114.9$. HRMS (DART) $\mathrm{m} / \mathrm{z}$ calcd. for $\mathrm{C}_{17} \mathrm{H}_{19} \mathrm{FO}_{2}^{-}[\mathrm{M}-\mathrm{H}]^{-}:$273.1296; 273.1293 found. IR (thin film) 3409, 2874, 1602, 1508, $1219,1114,835,700 \mathrm{~cm}^{-1}$. Specific rotation $[\alpha]_{\mathrm{D}}{ }^{24}=+28.3\left(\mathrm{c}=1.0, \mathrm{CHCl}_{3}\right)$. HPLC analysis: Chiralcel OD-H (Hex/IPA =95/5, $\left.1.0 \mathrm{~mL} / \mathrm{min}, 210 \mathrm{~nm}, 23^{\circ} \mathrm{C}\right), 14.5 \mathrm{~min}$ (major), $16.2 \mathrm{~min}$ (minor), $85 \%$ ee.

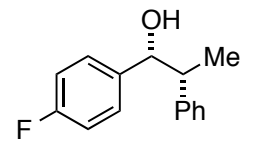

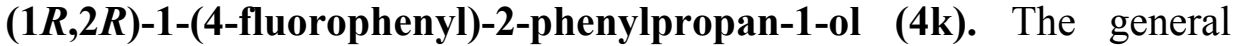
procedure was followed using styrene $(114 \mu \mathrm{L}, 1.0 \mathrm{mmol}, 1.0$ equiv) and 4-fluorobenzoic anhydride (393 mg, $1.5 \mathrm{mmol}, 1.5$ equiv) with a $7 \mathrm{~h}$ reaction time. ${ }^{1} \mathrm{H}$ NMR analysis of the crude reaction material indicated $>20: 1 \mathrm{dr}$. Silica gel column chromatography ( 0 to $10 \% \mathrm{Et}_{2} \mathrm{O}$ in hexanes) yielded the title product as a white solid (Run 1: $185 \mathrm{mg}, 0.803 \mathrm{mmol}, 80 \%$ yield; Run 2: $201 \mathrm{mg}, 0.873$ mmol, 87\% yield). ${ }^{1} \mathbf{H}$ NMR $\left(400 \mathrm{MHz}, \mathrm{CDCl}_{3}\right) \delta 7.47-7.21(\mathrm{~m}, 7 \mathrm{H}), 7.07(\mathrm{t}, \mathrm{J}=8.7$ $\mathrm{Hz}, 2 \mathrm{H}), 4.67(\mathrm{dd}, \mathrm{J}=8.6,2.3 \mathrm{~Hz}, 1 \mathrm{H}), 3.00(\mathrm{dq}, \mathrm{J}=8.7,7.1 \mathrm{~Hz}, 1 \mathrm{H}), 1.88(\mathrm{~d}, \mathrm{~J}=2.3 \mathrm{~Hz}$, $1 \mathrm{H}), 1.10(\mathrm{~d}, \mathrm{~J}=7.0 \mathrm{~Hz}, 3 \mathrm{H}) .{ }^{13} \mathbf{C}$ NMR $\left(101 \mathrm{MHz}, \mathrm{CDCl}_{3}\right) \delta 163.7,161.3,143.2,138.4$, $138.3,128.9,128.7,128.6,128.2,127.2,115.4,115.1,79.1,48.4,18.3$. m.p. $79-81{ }^{\circ} \mathrm{C}$. ${ }^{19}$ F NMR $\left(376 \mathrm{MHz}, \mathrm{CDCl}_{3}\right) \delta$-114.8. IR (thin film) 3417, 2965, 1602, 1509, 1221, 1022, 835, 751, $700 \mathrm{~cm}^{-1}$. EA Calcd. for $\mathrm{C}_{15} \mathrm{H}_{15} \mathrm{FO}$ : C, 78.24; $\mathrm{H}, 6.57$. Found: C, 77.98; $\mathrm{H}$, 6.73. Specific rotation $[\alpha]_{\mathrm{D}}{ }^{24}=+58.5\left(\mathrm{c}=1.0, \mathrm{CHCl}_{3}\right)$. HPLC analysis: Chiralpak $\mathrm{AD}-\mathrm{H}\left(\mathrm{Hex} / \mathrm{IPA}=95 / 5,1.0 \mathrm{~mL} / \mathrm{min}, 210 \mathrm{~nm}, 23^{\circ} \mathrm{C}\right), 11.2 \mathrm{~min}$ (minor), $12.5 \mathrm{~min}$ (major), 97\% ee.

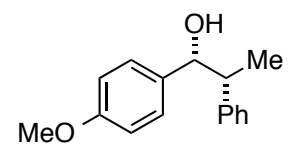

(1R,2R)-1-(4-methoxyphenyl)-2-phenylpropan-1-ol (4I). For this substrate, $4 \mathrm{~mol} \%$ catalyst consisting of $\mathrm{Cu}(\mathrm{OAc})_{2}(7.2 \mathrm{mg}, 0.04$ mmol, 0.04 equiv), $(S, S)$-Ph-BPE (22.2 mg, $0.044 \mathrm{mmol}, 0.044$ equiv) and $\mathrm{PPh}_{3}$ (11.5 mg, $0.044 \mathrm{mmol}, 0.044$ equiv) was used. The general procedure was followed using styrene (114 $\mu \mathrm{L}, 1.0 \mathrm{mmol}, 1.0$ equiv) and 4- 
methoxybenzoic anhydride (429 mg, $1.5 \mathrm{mmol}, 1.5$ equiv) with a $60 \mathrm{~h}$ reaction time. ${ }^{1} \mathrm{H}$ NMR analysis of the crude reaction material indicated $6: 1 \mathrm{dr}$. Silica gel column chromatography (5 to $15 \% \mathrm{Et}_{2} \mathrm{O}$ in hexanes) yielded the title product as a white solid (Run 1: $142 \mathrm{mg}, 0.587 \mathrm{mmol}, 59 \%$ yield; Run 2: $157 \mathrm{mg}, 0.648 \mathrm{mmol}, 65 \%$ yield). ${ }^{1} \mathbf{H}$ NMR $\left(400 \mathrm{MHz}, \mathrm{CDCl}_{3}\right) \delta 7.50-7.24(\mathrm{~m}, 7 \mathrm{H}), 6.93(\mathrm{~d}, \mathrm{~J}=8.7 \mathrm{~Hz}, 2 \mathrm{H}), 4.65(\mathrm{~d}, \mathrm{~J}=8.8$ $\mathrm{Hz}, 1 \mathrm{H}), 3.85(\mathrm{~s}, 3 \mathrm{H}), 3.10-2.94(\mathrm{~m}, 1 \mathrm{H}), 1.82(\mathrm{~s}, 1 \mathrm{H}), 1.09(\mathrm{~d}, \mathrm{~J}=7.1 \mathrm{~Hz}, 3 \mathrm{H}) .{ }^{13} \mathbf{C}$ NMR $\left(101 \mathrm{MHz}, \mathrm{CDCl}_{3}\right) \delta 159.3,143.7,134.8,128.8,128.2,128.1,126.9,113.8,79.3$, 55.4, 48.3, 18.5. HRMS (DART) $\mathrm{m} / \mathrm{z}$ calcd. for $\mathrm{C}_{16} \mathrm{H}_{17} \mathrm{O}_{2}^{-}[\mathrm{M}-\mathrm{H}]^{-}: 241.1234 ; 241.1256$ found. m.p. $69-71{ }^{\circ} \mathrm{C}$. IR (thin film) 3413, 2952, 1610, 1510, 1451, 1247, 1179, 829, 700 $\mathrm{cm}^{-1}$. Specific rotation $[\alpha]_{\mathrm{D}}{ }^{24}=+47.5\left(\mathrm{c}=1.0, \mathrm{CHCl}_{3}\right)$. HPLC analysis: Chiralpak AD-H $\left(\mathrm{Hex} / \mathrm{IPA}=95 / 5,1.0 \mathrm{~mL} / \mathrm{min}, 230 \mathrm{~nm}, 23^{\circ} \mathrm{C}\right.$ ), $20.5 \mathrm{~min}$ (major), $25.5 \mathrm{~min}$ (minor), 83\% ee.

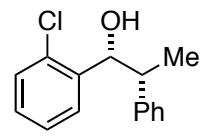

(1R,2R)-1-(2-chlorophenyl)-2-phenylpropan-1-ol (4m). For this substrate, $4 \mathrm{~mol} \%$ catalyst consisting of $\mathrm{Cu}(\mathrm{OAc})_{2}(7.2 \mathrm{mg}, 0.04 \mathrm{mmol}$, 0.04 equiv), $(S, S)$-Ph-BPE (22.2 mg, $0.044 \mathrm{mmol}, 0.044$ equiv) and $\mathrm{PPh}_{3}$ (11.5 mg, $0.044 \mathrm{mmol}, 0.044$ equiv) was used. The general procedure was followed using styrene $(114 \mu \mathrm{L}, 1.0 \mathrm{mmol}, 1.0$ equiv) and 2-chlorobenzoic anhydride (442 $\mathrm{mg}, 1.5$ mmol, 1.5 equiv) with a $36 \mathrm{~h}$ reaction time. ${ }^{1} \mathrm{H} \mathrm{NMR}$ analysis of the crude reaction material indicated 10:1 dr. Silica gel column chromatography (0 to $10 \% \mathrm{Et}_{2} \mathrm{O}$ in hexanes) yielded the title product as a colorless viscous oil (Run 1: $193 \mathrm{mg}, 0.782 \mathrm{mmol}, 78 \%$ yield; Run 2: $208 \mathrm{mg}, 0.843 \mathrm{mmol}, 84 \%$ yield). ${ }^{1} \mathbf{H}$ NMR $\left(400 \mathrm{MHz}, \mathrm{CDCl}_{3}\right) \delta 7.48-$ $7.13(\mathrm{~m}, 9 \mathrm{H}), 5.31(\mathrm{dd}, \mathrm{J}=7.8,3.5 \mathrm{~Hz}, 1 \mathrm{H}), 3.12$ (quintet, $\mathrm{J}=7.3 \mathrm{~Hz}, 1 \mathrm{H}), 1.89(\mathrm{~d}, \mathrm{~J}=$ $3.7 \mathrm{~Hz}, 1 \mathrm{H}), 1.25(\mathrm{~d}, \mathrm{~J}=7.2 \mathrm{~Hz}, 3 \mathrm{H}) .{ }^{13} \mathbf{C} \mathbf{N M R}\left(101 \mathrm{MHz}, \mathrm{CDCl}_{3}\right) \delta 142.8,140.5$, $133.0,129.4,128.7,128.7,128.4,128.2,127.1,127.1,74.8,47.8,18.2$. IR (thin film) 3408, 2975, 1451, 1439, 1025, 750, $697 \mathrm{~cm}^{-1}$. EA Calcd. for $\mathrm{C}_{15} \mathrm{H}_{15} \mathrm{ClO}: \mathrm{C}, 73.02 ; \mathrm{H}$, 6.13. Found: $\mathrm{C}, 73.05 ; \mathrm{H}, 6.26$. Specific rotation $[\alpha]_{\mathrm{D}}{ }^{24}=+62.3\left(\mathrm{c}=1.0, \mathrm{CHCl}_{3}\right)$. HPLC analysis: Chiralpak AD-H (Hex/IPA =97/3, $\left.1.0 \mathrm{~mL} / \mathrm{min}, 210 \mathrm{~nm}, 23^{\circ} \mathrm{C}\right), 14.2 \mathrm{~min}$ (major), $15.6 \mathrm{~min}$ (minor), $82 \%$ ee.

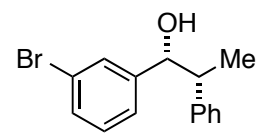

(1R,2R)-1-(3-bromophenyl)-2-phenylpropan-1-ol (4n). For this substrate, $4 \mathrm{~mol} \%$ catalyst consisting of $\mathrm{Cu}(\mathrm{OAc})_{2}(7.2 \mathrm{mg}, 0.04 \mathrm{mmol}$, 0.04 equiv), $(S, S)-\mathrm{Ph}-\mathrm{BPE}(22.2 \mathrm{mg}, 0.044 \mathrm{mmol}, 0.044$ equiv) and $\mathrm{PPh}_{3}(11.5 \mathrm{mg}, 0.044 \mathrm{mmol}, 0.044$ equiv) was used. The general procedure was followed using styrene (114 $\mu \mathrm{L}, 1.0 \mathrm{mmol}, 1.0$ equiv) and 3-bromobenzoic anhydride (768 mg, 2.0 mmol, 2.0 equiv) with a $36 \mathrm{~h}$ reaction time. ${ }^{1} \mathrm{H}$ NMR analysis of the crude reaction material indicated $>20: 1 \mathrm{dr}$. Silica gel column chromatography (15\% $\mathrm{Et}_{2} \mathrm{O}$ in hexanes) yielded the title product as a white solid (Run 1: $186 \mathrm{mg}, 0.639 \mathrm{mmol}, 64 \%$ yield; Run 2: $188 \mathrm{mg}, 0.646 \mathrm{mmol}, 65 \%$ yield). ${ }^{1} \mathbf{H}$ NMR $\left(400 \mathrm{MHz}, \mathrm{CDCl}_{3}\right) \delta 7.55$ (t, J = $\left.1.8 \mathrm{~Hz}, 1 \mathrm{H}\right)$, $7.52-7.18(\mathrm{~m}, 8 \mathrm{H}), 4.64(\mathrm{dd}, \mathrm{J}=8.6,2.4 \mathrm{~Hz}, 1 \mathrm{H}), 3.09-2.90(\mathrm{~m}, 1 \mathrm{H}), 1.90(\mathrm{~d}, \mathrm{~J}=2.5$ $\mathrm{Hz}, 1 \mathrm{H}), 1.12(\mathrm{~d}, \mathrm{~J}=7.1 \mathrm{~Hz}, 3 \mathrm{H}) .{ }^{13} \mathbf{C} \mathbf{N M R}\left(101 \mathrm{MHz}, \mathrm{CDCl}_{3}\right) \delta 145.0,142.9,131.0$, $130.2,129.9,128.9,128.2,127.3,125.9,122.6,79.2,48.3,18.3$. HRMS (DART) $\mathrm{m} / \mathrm{z}$ calcd. for $\mathrm{C}_{15} \mathrm{H}_{19} \mathrm{BrNO}^{+}\left[\mathrm{M}+\mathrm{NH}_{4}\right]^{+}: 308.0645 ; 308.0658$ found. m.p. $42-44{ }^{\circ} \mathrm{C}$. IR (thin film) $3354,2970,1197,1036,873,774,692 \mathrm{~cm}^{-1}$. Specific rotation $[\alpha]_{\mathrm{D}}{ }^{24}=+47.4(\mathrm{c}=$ 
1.0, $\left.\mathrm{CHCl}_{3}\right)$. HPLC analysis: Chiralpak AD-H (Hex/IPA = 97/3, $1.0 \mathrm{~mL} / \mathrm{min}, 210 \mathrm{~nm}$, $23^{\circ} \mathrm{C}$ ), 18.4 min (minor), 21.4 min (major), 95\% ee.

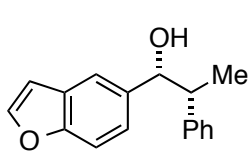

(1R,2R)-1-(benzofuran-5-yl)-2-phenylpropan-1-ol (40). For this substrate, $4 \mathrm{~mol} \%$ catalyst consisting of $\mathrm{Cu}(\mathrm{OAc})_{2}(7.2 \mathrm{mg}, 0.04 \mathrm{mmol}$, 0.04 equiv), (S,S)-Ph-BPE (22.2 $\mathrm{mg}, 0.044 \mathrm{mmol}, 0.044$ equiv) and $\mathrm{PPh}_{3}$ (11.5 mg, $0.044 \mathrm{mmol}, 0.044$ equiv) was used. The general procedure was followed using styrene (114 $\mu \mathrm{L}, 1.0 \mathrm{mmol}, 1.0$ equiv) and benzofuran-5carboxylic anhydride (459 mg, $1.5 \mathrm{mmol}, 1.5$ equiv) with a $24 \mathrm{~h}$ reaction time. ${ }^{1} \mathrm{H} \mathrm{NMR}$ analysis of the crude reaction material indicated 12:1 dr. Silica gel column chromatography (10 to $20 \% \mathrm{Et}_{2} \mathrm{O}$ in hexanes) yielded the title product as a white solid (Run 1: $211 \mathrm{mg}, 0.836 \mathrm{mmol}, 84 \%$ yield; Run 2: $200 \mathrm{mg}, 0.793 \mathrm{mmol}, 79 \%$ yield). ${ }^{1} \mathbf{H}$ NMR $\left(400 \mathrm{MHz}, \mathrm{CDCl}_{3}\right) \delta 7.66(\mathrm{dd}, \mathrm{J}=9.7,2.0 \mathrm{~Hz}, 2 \mathrm{H}), 7.56-7.50(\mathrm{~m}, 1 \mathrm{H}), 7.46-$ $7.30(\mathrm{~m}, 6 \mathrm{H}), 6.80(\mathrm{dd}, \mathrm{J}=2.2,1.0 \mathrm{~Hz}, 1 \mathrm{H}), 4.79(\mathrm{dd}, \mathrm{J}=8.9,2.2 \mathrm{~Hz}, 1 \mathrm{H}), 3.16-3.01$ $(\mathrm{m}, 1 \mathrm{H}), 1.92(\mathrm{~d}, \mathrm{~J}=2.2 \mathrm{~Hz}, 1 \mathrm{H}), 1.10(\mathrm{~d}, \mathrm{~J}=7.1 \mathrm{~Hz}, 3 \mathrm{H}) .{ }^{\mathbf{1 3}} \mathbf{C}$ NMR $\left(101 \mathrm{MHz}, \mathrm{CDCl}_{3}\right)$ $\delta 154.8,145.5,143.7,137.4,128.9,128.2,127.5,127.1,123.5,119.8,111.3,106.8,80.0$, 48.7, 18.7. HRMS (DART) $\mathrm{m} / \mathrm{z}$ calcd. for $\mathrm{C}_{17} \mathrm{H}_{15} \mathrm{O}_{2}{ }^{-}[\mathrm{M}-\mathrm{H}]^{-}: 251.1078 ; 251.1091$ found. m.p. 59-61 ${ }^{\circ} \mathrm{C}$. IR (thin film) 3396, 2959, 1452, 1263, 1124, 1027, 742, $699 \mathrm{~cm}^{-1}$. Specific rotation $[\alpha]_{\mathrm{D}}{ }^{24}=+44.1\left(\mathrm{c}=1.0, \mathrm{CHCl}_{3}\right)$. HPLC analysis: Chiralpak AD-H $\left(\mathrm{Hex} / \mathrm{IPA}=98 / 2,1.0 \mathrm{~mL} / \mathrm{min}, 210 \mathrm{~nm}, 23^{\circ} \mathrm{C}\right.$ ), $51.1 \mathrm{~min}$ (major), $53.8 \mathrm{~min}$ (minor), 90\% ee.

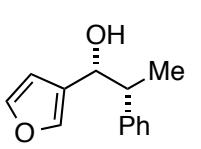

(1R,2R)-1-(furan-3-yl)-2-phenylpropan-1-ol (4p). The general procedure was followed using styrene (114 $\mu \mathrm{L}, 1.0 \mathrm{mmol}, 1.0$ equiv) and furan-3carboxylic anhydride (416 $\mathrm{mg}, 2.0 \mathrm{mmol}, 2.0$ equiv) with a $36 \mathrm{~h}$ reaction time. ${ }^{1} \mathrm{H}$ NMR analysis of the crude reaction material indicated $>20: 1 \mathrm{dr}$. Silica gel column chromatography ( 0 to $10 \% \mathrm{Et}_{2} \mathrm{O}$ in hexanes) yielded the title product as a white solid (Run 1: $116 \mathrm{mg}, 0.574 \mathrm{mmol}, 57 \%$ yield; Run 2: $128 \mathrm{mg}, 0.633 \mathrm{mmol}, 63 \%$ yield). ${ }^{1} \mathbf{H}$ NMR $\left(400 \mathrm{MHz}, \mathrm{CDCl}_{3}\right) \delta 7.48-7.23(\mathrm{~m}, 7 \mathrm{H}), 6.50-6.34(\mathrm{~m}, 1 \mathrm{H}), 4.78-$ $4.69(\mathrm{~m}, 1 \mathrm{H}), 3.03(\mathrm{dd}, \mathrm{J}=8.2,7.0 \mathrm{~Hz}, 1 \mathrm{H}), 1.76(\mathrm{~d}, \mathrm{~J}=2.4 \mathrm{~Hz}, 1 \mathrm{H}), 1.20(\mathrm{~d}, \mathrm{~J}=7.0 \mathrm{~Hz}$, $3 \mathrm{H}) .{ }^{13} \mathrm{C}$ NMR $\left(101 \mathrm{MHz}, \mathrm{CDCl}_{3}\right) \delta 143.4,143.2,140.2,128.8,128.2,127.1,127.0$, 108.8, 72.0, 47.2, 18.3. HRMS (DART) m/z calcd. for $\mathrm{C}_{13} \mathrm{H}_{18} \mathrm{NO}_{2}^{+}\left[\mathrm{M}+\mathrm{NH}_{4}\right]^{+}$: 220.1332; 220.1334 found. m.p. 46-48 ${ }^{\circ}$ C. IR (thin film) 3404, 2964, 1495, 1157, 1092 , $872,781,699 \mathrm{~cm}^{-1}$. Specific rotation $[\alpha]_{\mathrm{D}}{ }^{24}=+36.7\left(\mathrm{c}=1.0, \mathrm{CHCl}_{3}\right)$. HPLC analysis: Chiralcel OD-H (Hex/IPA = 95/5, $1.0 \mathrm{~mL} / \mathrm{min}, 210 \mathrm{~nm}, 23^{\circ} \mathrm{C}$ ), $11.9 \mathrm{~min}$ (major), 14.1 $\min$ (minor), $93 \%$ ee.

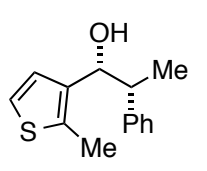

(1R,2R)-1-(2-methylthiophen-3-yl)-2-phenylpropan-1-ol (4q). For this substrate, $4 \mathrm{~mol} \%$ catalyst consisting of $\mathrm{Cu}(\mathrm{OAc})_{2}(7.2 \mathrm{mg}, 0.04 \mathrm{mmol}$, 0.04 equiv), $(S, S)$-Ph-BPE (22.2 mg, 0.044 mmol, 0.044 equiv) and $\mathrm{PPh}_{3}$ (11.5 mg, $0.044 \mathrm{mmol}, 0.044$ equiv) was used. The general procedure was followed using styrene (114 $\mu \mathrm{L}, 1.0 \mathrm{mmol}, 1.0$ equiv) and 2-methylthiophene-3carboxylic anhydride (399 mg, $1.5 \mathrm{mmol}, 1.5$ equiv) with a $48 \mathrm{~h}$ reaction time. ${ }^{1} \mathrm{H}$ NMR analysis of the crude reaction material indicated 10:1 dr. Silica gel column chromatography (10 to $20 \% \mathrm{Et}_{2} \mathrm{O}$ in hexanes) yielded the title product as a white solid 
(Run 1: $167 \mathrm{mg}, 0.719 \mathrm{mmol}, 72 \%$ yield; Run 2: $170 \mathrm{mg}, 0.732 \mathrm{mmol}, 73 \%$ yield). ${ }^{1} \mathbf{H}$ NMR $\left(400 \mathrm{MHz}, \mathrm{CDCl}_{3}\right) \delta 7.48-7.26(\mathrm{~m}, 5 \mathrm{H}), 7.15-7.03(\mathrm{~m}, 2 \mathrm{H}), 4.84(\mathrm{dd}, \mathrm{J}=8.9$, $2.3 \mathrm{~Hz}, 1 \mathrm{H}), 3.11(\mathrm{dq}, \mathrm{J}=8.8,7.1 \mathrm{~Hz}, 1 \mathrm{H}), 2.42(\mathrm{~s}, 3 \mathrm{H}), 1.77(\mathrm{~d}, \mathrm{~J}=2.4 \mathrm{~Hz}, 1 \mathrm{H}), 1.13$ (d, $\mathrm{J}=7.1 \mathrm{~Hz}, 3 \mathrm{H}) .{ }^{13} \mathbf{C} \mathbf{N M R}\left(101 \mathrm{MHz}, \mathrm{CDCl}_{3}\right) \delta 143.6,138.5,135.5,128.9,128.1,127.1$, 126.4, 122.1, 73.3, 47.9, 17.9, 13.2. HRMS (DART) m/z calcd. for $\mathrm{C}_{14} \mathrm{H}_{15} \mathrm{OS}^{-}[\mathrm{M}-\mathrm{H}]^{-}$: $231.0849 ; 231.0830$ found. m.p. $72-74{ }^{\circ} \mathrm{C}$. Specific rotation $[\alpha]_{\mathrm{D}}{ }^{24}=+9.0(\mathrm{c}=1.0$, $\left.\mathrm{CHCl}_{3}\right)$. HPLC analysis: Chiralpak AD-H (Hex/IPA = 97/3, $\left.1.0 \mathrm{~mL} / \mathrm{min}, 210 \mathrm{~nm}, 23^{\circ} \mathrm{C}\right)$, 13.5 min (major), 16.4 min (minor), $88 \%$ ee.

\section{Ketone Synthesis: General Procedure and Substrate Scope}

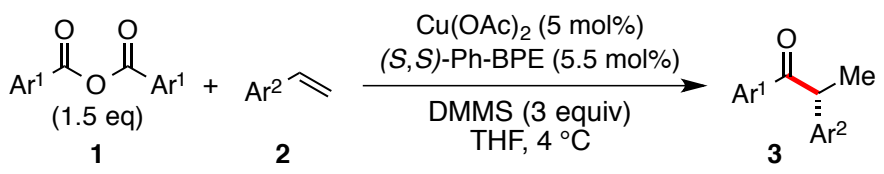

General procedure. An oven dried reaction tube (Fisherbrand, 16 x $125 \mathrm{~mm}$, catalog no. 1495925C) containing a magnetic stir bar was charged with aryl carboxylic acid anhydride (1.5 mmol, 1.5 equiv). The reaction tube was loosely capped (Cap: Kimble Chase Open Top S/T Closure catalog no. 73804-15425; Septum: Thermo Scientific 2.6 mm silicone/PTFE catalog no. B7995-15), then brought into a nitrogen filled glove box. $\mathrm{Cu}(\mathrm{OAc})_{2}(9.1 \mathrm{mg}, 0.05 \mathrm{mmol}, 0.05$ equiv) and $(S, S)-\mathrm{Ph}-\mathrm{BPE}(28 \mathrm{mg}, 0.055 \mathrm{mmol}, 0.055$ equiv) were then added to the reaction tube. The reaction tube was capped, removed from the glove box, and put under a positive pressure of nitrogen via a needle line connected to a Schlenk line. THF (2 mL) was added via a syringe under nitrogen to the reaction tube. The resulting mixture was stirred for 10 min until a deep blue homogenous solution formed. The alkene was added via microsyringe at this time (1.0 mmol, 1.0 equiv). DMMS (369 $\mu \mathrm{L}, 3.0 \mathrm{mmol}, 3.0$ equiv) was then added via a syringe under nitrogen and the resulting solution was stirred at $\mathrm{rt}$ for an additional $2 \mathrm{~min}$ as the solution turned to a yellow, orange or red color. The nitrogen needle line was removed, the cap wrapped in parafilm, and the reaction tube was inserted into a refrigerator kept at $4{ }^{\circ} \mathrm{C}$. After the time indicated for each reaction, the reaction tube was removed from the refrigerator and slowly quenched with sat. aqueous $\mathrm{NaHCO}_{3}(5 \mathrm{~mL}$, Caution: gas evolution observed). The mixture was extracted with EtOAc $(3 \times 5 \mathrm{~mL})$ and the organic extracts were dried with anhydrous $\mathrm{Na}_{2} \mathrm{SO}_{4}$, filtered, concentrated in vacuo and the crude material was purified by silica gel column chromatography.

O (R)-1,2-diphenylpropan-1-one (3a). The general procedure was followed using styrene (114 $\mu \mathrm{L}, 1.0 \mathrm{mmol}, 1.0$ equiv) and benzoic anhydride (339 $\mathrm{mg}$, 1.5 mmol, 1.5 equiv) with a $12 \mathrm{~h}$ reaction time. Silica gel column chromatography ( 0 to $3 \% \mathrm{Et}_{2} \mathrm{O}$ in hexanes) yielded the title product as a colorless oil (Run 1: $164 \mathrm{mg}, 0.780 \mathrm{mmol}, 78 \%$ yield; Run 2: $169 \mathrm{mg}, 0.804 \mathrm{mmol}, 80 \%$ yield). ${ }^{1} \mathbf{H}$ NMR $\left(400 \mathrm{MHz}, \mathrm{CDCl}_{3}\right) \delta 8.08-7.90(\mathrm{~m}, 2 \mathrm{H}), 7.49(\mathrm{~d}, \mathrm{~J}=7.3 \mathrm{~Hz}, 1 \mathrm{H}), 7.41(\mathrm{dd}, \mathrm{J}=$ $8.2,6.8 \mathrm{~Hz}, 2 \mathrm{H}), 7.36-7.18(\mathrm{~m}, 5 \mathrm{H}), 4.72(\mathrm{q}, \mathrm{J}=6.8 \mathrm{~Hz}, 1 \mathrm{H}), 1.57(\mathrm{~d}, \mathrm{~J}=6.8 \mathrm{~Hz}, 3 \mathrm{H})$. ${ }^{13}$ C NMR $\left(101 \mathrm{MHz}, \mathrm{CDCl}_{3}\right) \delta 200.5,141.6,136.6,132.9,129.1,128.9,128.61,127.9$, 127.0, 48.0, 19.7. HRMS (DART) m/z calcd. for $\mathrm{C}_{15} \mathrm{H}_{15} \mathrm{O}^{+}[\mathrm{M}+\mathrm{H}]^{+}: 211.1117 ; 211.2835$ 
found. IR (thin film) 2975, 1679, 1596, 1447, 1219, 951, $695 \mathrm{~cm}^{-1}$. Specific rotation $[\alpha]_{\mathrm{D}}^{24}=-147.0\left(\mathrm{c}=1.0, \mathrm{CHCl}_{3}\right)$. HPLC analysis: Chiralpak AD-H $(\mathrm{Hex} / \mathrm{IPA}=98 / 2,0.8$ $\mathrm{mL} / \mathrm{min}, 254 \mathrm{~nm}, 23^{\circ} \mathrm{C}$ ), $8.3 \mathrm{~min}$ (minor), $9.0 \mathrm{~min}$ (major), $89 \%$ ee. The absolute stereochemistry was determined by comparison of optical rotation measurement and HPLC retention times with literature data.

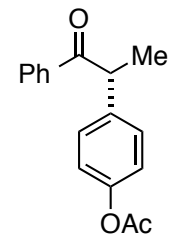

(R)-4-(1-oxo-1-phenylpropan-2-yl)phenyl acetate (3b). The general procedure was followed using 4-acetoxystyrene $(153 \mu \mathrm{L}, 1.0 \mathrm{mmol}, 1.0$ equiv) and benzoic anhydride (339 $\mathrm{mg}, 1.5 \mathrm{mmol}, 1.5$ equiv) with an $8 \mathrm{~h}$ reaction time. Silica gel column chromatography ( 0 to $10 \% \mathrm{Et}_{2} \mathrm{O}$ in hexanes) yielded the title product as a white solid (Run 1: $183 \mathrm{mg}, 0.682 \mathrm{mmol}, 68 \%$ yield; Run 2: $168 \mathrm{mg}, 0.626 \mathrm{mmol}, 63 \%$ yield). ${ }^{1} \mathbf{H}$ NMR $\left(400 \mathrm{MHz}, \mathrm{CDCl}_{3}\right)$ $\delta 8.02-7.91(\mathrm{~m}, 2 \mathrm{H}), 7.51(\mathrm{~d}, \mathrm{~J}=7.4 \mathrm{~Hz}, 1 \mathrm{H}), 7.46-7.38(\mathrm{~m}, 2 \mathrm{H}), 7.36-7.23(\mathrm{~m}, 2 \mathrm{H})$, $7.07-7.00(\mathrm{~m}, 2 \mathrm{H}), 4.73(\mathrm{q}, \mathrm{J}=6.9 \mathrm{~Hz}, 1 \mathrm{H}), 2.29(\mathrm{~s}, 3 \mathrm{H}), 1.55(\mathrm{~d}, \mathrm{~J}=6.9 \mathrm{~Hz}, 3 \mathrm{H}) .{ }^{13} \mathbf{C}$ NMR $\left(101 \mathrm{MHz}, \mathrm{CDCl}_{3}\right) \delta 200.2,169.4,149.6,138.9,136.4,133.0,128.9,128.82$, 128.6, 122.1, 47.1, 21.2, 19.6. HRMS (DART) m/z calcd. for $\mathrm{C}_{17} \mathrm{H}_{17} \mathrm{O}_{3}{ }^{+}[\mathrm{M}+\mathrm{H}]^{+}$: 269.1172; 269.1179 found. m.p. $75-77^{\circ} \mathrm{C}$. IR (thin film) 2979, 1760, 1672, 1197, 1170, 910, $685 \mathrm{~cm}^{-1}$. Specific rotation $[\alpha]_{\mathrm{D}}{ }^{24}=-110.8\left(\mathrm{c}=1.0, \mathrm{CHCl}_{3}\right)$. HPLC analysis: Chiralpak AD-H (Hex/IPA = 96/4, $1.0 \mathrm{~mL} / \mathrm{min}, 210 \mathrm{~nm}, 23^{\circ} \mathrm{C}$ ), $11.7 \mathrm{~min}$ (minor), 13.6 min (major), $97 \%$ ee.

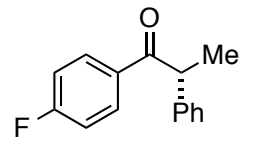

(R)-1-(4-fluorophenyl)-2-phenylpropan-1-one (3c). The general procedure was followed using styrene $(114 \mu \mathrm{L}, 1.0 \mathrm{mmol}, 1.0$ equiv) and 4-fluorobenzoic anhydride (393 mg, $1.5 \mathrm{mmol}, 1.5$ equiv) with an $8 \mathrm{~h}$ reaction time. Silica gel column chromatography $\left(0\right.$ to $2 \% \mathrm{Et}_{2} \mathrm{O}$ in hexanes) yielded the title product as a colorless oil (Run 1: $175 \mathrm{mg}, 0.767 \mathrm{mmol}, 77 \%$ yield; Run 2: $174 \mathrm{mg}, 0.762 \mathrm{mmol}, 76 \%$ yield). ${ }^{1} \mathbf{H}$ NMR $\left(400 \mathrm{MHz}, \mathrm{CDCl}_{3}\right) \delta 8.07-$ $7.94(\mathrm{~m}, 2 \mathrm{H}), 7.40-7.18(\mathrm{~m}, 5 \mathrm{H}), 7.06(\mathrm{t}, \mathrm{J}=8.6 \mathrm{~Hz}, 2 \mathrm{H}), 4.65(\mathrm{q}, \mathrm{J}=6.8 \mathrm{~Hz}, 1 \mathrm{H}), 1.55$ $(\mathrm{d}, \mathrm{J}=6.8 \mathrm{~Hz}, 3 \mathrm{H}) .{ }^{13} \mathrm{C}$ NMR $\left(101 \mathrm{MHz}, \mathrm{CDCl}_{3}\right) \delta 198.8,166.8,164.3,141.5,133.0$, $132.9,131.6,131.5,129.2,127.8,127.1,115.8,115.6,48.1,19.6 .{ }^{19} \mathbf{F}$ NMR $(376 \mathrm{MHz}$, $\left.\mathrm{CDCl}_{3}\right) \delta$-105.6. HRMS (DART) $\mathrm{m} / \mathrm{z}$ calcd. for $\mathrm{C}_{15} \mathrm{H}_{14} \mathrm{FO}^{+}[\mathrm{M}+\mathrm{H}]^{+}$: 229.1023; 229.1009 found. IR (thin film) 2977, 1680, 1596, 1223, 1155, 952, 850, $699 \mathrm{~cm}^{-1}$. Specific rotation $[\alpha]_{\mathrm{D}}{ }^{24}=-161.9\left(\mathrm{c}=1.0, \mathrm{CHCl}_{3}\right)$. HPLC analysis: Chiralcel OD-H $\left(\mathrm{Hex} / \mathrm{IPA}=95 / 5,1.0 \mathrm{~mL} / \mathrm{min}, 230 \mathrm{~nm}, 23^{\circ} \mathrm{C}\right.$ ), $5.2 \mathrm{~min}$ (minor), $5.8 \mathrm{~min}$ (major), 79\% ee.

$(\boldsymbol{R})-1-($ furan-3-yl)-2-phenylpropan-1-one $(\mathbf{3 d})$. For this substrate, the
reaction was run at rt. The general procedure was followed using styrene $1.5 \mathrm{mmol}, 1.5$ equiv) with a $7 \mathrm{~h}$ reaction time. Silica gel column chromatography $(2 \%$ $\mathrm{Et}_{2} \mathrm{O}$ in hexanes) yielded the title product as a white solid (Run 1: $145 \mathrm{mg}, 0.720 \mathrm{mmol}$, $72 \%$ yield; Run 2: $149 \mathrm{mg}, 0.744 \mathrm{mmol}, 74 \%$ yield). ${ }^{1} \mathbf{H}$ NMR $\left(400 \mathrm{MHz}, \mathrm{CDCl}_{3}\right) \delta 7.90$ $(\mathrm{t}, \mathrm{J}=1.1 \mathrm{~Hz}, 1 \mathrm{H}), 7.42-7.18(\mathrm{~m}, 6 \mathrm{H}), 6.73(\mathrm{dd}, \mathrm{J}=1.9,0.9 \mathrm{~Hz}, 1 \mathrm{H}), 4.25(\mathrm{q}, \mathrm{J}=6.9$ $\mathrm{Hz}, 1 \mathrm{H}), 1.53(\mathrm{dd}, \mathrm{J}=6.9,1.4 \mathrm{~Hz}, 3 \mathrm{H}) .{ }^{13} \mathbf{C} \mathbf{N M R}\left(101 \mathrm{MHz}, \mathrm{CDCl}_{3}\right) \delta 195.3,147.7$, $143.9,141.5,129.1,127.8,127.2,127.1,109.3,50.8,18.9$. m.p. $35-37{ }^{\circ} \mathrm{C}$. IR (thin film) $3130,1671,1510,1153,1023,907,872,696 \mathrm{~cm}^{-1}$. EA Calcd. for $\mathrm{C}_{12} \mathrm{H}_{12} \mathrm{O}_{2}: \mathrm{C}, 77.98 ; \mathrm{H}$, 6.04. Found: $\mathrm{C}, 77.91 ; \mathrm{H}, 6.19$. Specific rotation $[\alpha]_{\mathrm{D}}{ }^{24}=-129.6\left(\mathrm{c}=1.0, \mathrm{CHCl}_{3}\right)$. 
HPLC analysis: Chiralpak AD-H (Hex/IPA = 96/4, $\left.1.0 \mathrm{~mL} / \mathrm{min}, 210 \mathrm{~nm}, 23^{\circ} \mathrm{C}\right), 14.4$ min (minor), 15.2 min (major), $77 \%$ ee.

\section{Diastereoselective Reduction of Chiral Ketone 3a}
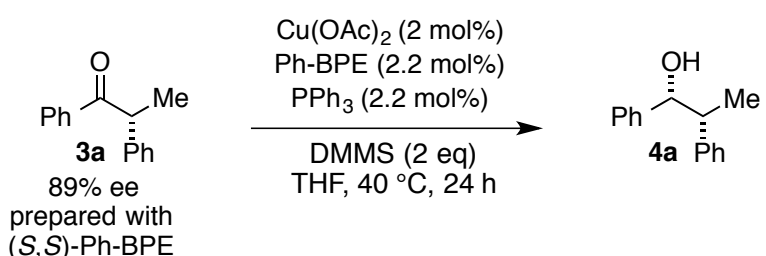

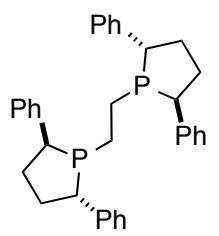

$(S, S)-\mathrm{Ph}-\mathrm{BPE}$

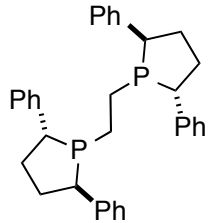

$(R, R)-\mathrm{Ph}-\mathrm{BPE}$

In a nitrogen filled glove box, $\mathrm{Cu}(\mathrm{OAc})_{2}(0.91 \mathrm{mg}, 0.005 \mathrm{mmol}, 0.02$ equiv), Ph-BPE ( $2.8 \mathrm{mg}, 0.0055 \mathrm{mmol}, 0.022$ equiv), $\mathrm{PPh}_{3}$ (1.4 mg, $0.0055 \mathrm{mmol}, 0.022$ equiv) and $\mathrm{THF}$ $(0.5 \mathrm{~mL})$ were added to an oven-dried reaction tube (Fisherbrand, $13 \times 100 \mathrm{~mm}$, catalog no. 1495925A) containing a magnetic stir bar. The mixture was stirred for 10 min until a homogenous blue solution formed, at which time ketone $3 \mathbf{a}(53 \mathrm{mg}, 0.25 \mathrm{mmol}, 1.0$ equiv) was added. After an additional 2 min of stirring, dimethoxymethylsilane $(62 \mu \mathrm{L}$, $0.50 \mathrm{mmol}, 2.0$ equiv) was added. The reaction solution turned an orange color. The reaction tube was capped (Thermo Scientific $13 \mathrm{~mm}$ screw cap with TEF/SIL septa, catalog no. C4015-66A), removed from the glove box, and inserted into an oil bath preheated to $40^{\circ} \mathrm{C}$. The reaction solution was stirred for $24 \mathrm{~h}$, at which time sat. aqueous $\mathrm{NaHCO}_{3}(3 \mathrm{~mL})$ was added to quench the reaction and the mixture was extracted with EtOAc $(3 \times 2 \mathrm{~mL})$. The combined extracted organic solution was dried with anhydrous $\mathrm{Na}_{2} \mathrm{SO}_{4}$, filtered and concentrated in vacuo. ${ }^{1} \mathrm{H} \mathrm{NMR}$ analysis of the crude material was used to determine the diastereomeric ratio by integration of the methyl groups of the product at $1.38 \mathrm{ppm}$ (minor) and $1.11 \mathrm{ppm}$ (major) and the $\mathrm{PhCHOH}$ proton at $4.95 \mathrm{ppm}$ (minor) and $4.85 \mathrm{ppm}$ (major). See below for ${ }^{1} \mathrm{H}$ NMR spectra of the crude reaction material.

For HPLC analysis, saturated $\mathrm{NH}_{4} \mathrm{~F}$ in $\mathrm{MeOH}$ was added to the crude reaction residue and the mixture was stirred for $30 \mathrm{~min}$. The mixture was concentrated in vacuo, and then partitioned between sat. aqueous $\mathrm{NaHCO}_{3}(5 \mathrm{~mL})$ and EtOAc $(5 \mathrm{~mL})$. The EtOAc layer was removed, dried with anhydrous $\mathrm{Na}_{2} \mathrm{SO}_{4}$, filtered and concentrated in vacuo. The crude material was purified by silica gel column chromatography $\left(10 \% \mathrm{Et}_{2} \mathrm{O}\right.$ in hexanes) to yield the desired product as a white solid. HPLC analysis: Chiralcel OD-H (Hex/IPA $=99 / 1,1.0 \mathrm{~mL} / \mathrm{min}, 210 \mathrm{~nm}, 23^{\circ} \mathrm{C}$ ), $25.3 \mathrm{~min}$ (minor diastereomer), $28.3 \mathrm{~min}$ (minor diastereomer), $30.0 \mathrm{~min}$ (major diastereomer) and $31.7 \mathrm{~min}$ (major diastereomer).

$(\boldsymbol{S}, \boldsymbol{S})$-Ph-BPE results: $100 \%{ }^{1} \mathrm{H}$ NMR yield; $97: 3 \mathrm{dr} ; 97 \%$ ee.

(R,R)-Ph-BPE results: $100 \%{ }^{1} \mathrm{H}$ NMR yield; 55:45 dr; 78\% and 99\% ee, respectively. 


\section{Preparation of Anhydride and Alkene Substrates}

Anhydrides: Benzoic anhydride, 4-fluorobenzoic anhydride and 4-methoxybenzoic anhydride were purchased from Alfa-Aeser and used as received. Furan-3-carboxylic anhydride $^{3}, 3$-bromobenzoic anhydride ${ }^{4}$ and 2 -chlorobenzoic anhydride ${ }^{5}$ were prepared according to literature procedure.

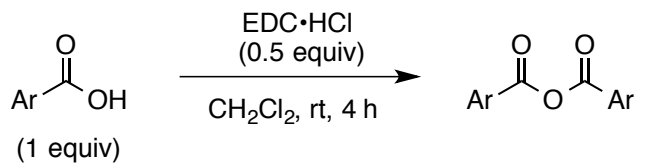

General procedure for anhydride synthesis. To an oven-dried round bottom flask containing a magnetic stir bar was added aryl carboxylic acid (1 equiv) and dry $\mathrm{CH}_{2} \mathrm{Cl}_{2}$ $([0.5 \mathrm{M}]) . \mathrm{N}$-(3-Dimethylaminopropyl)- $N$ '-ethylcarbodiimide hydrochloride $(\mathrm{EDC} \cdot \mathrm{HCl}$, 0.5 equiv) was then added and the reaction solution was stirred at $\mathrm{rt}$ for $4 \mathrm{~h}$. At this time, $\mathrm{H}_{2} \mathrm{O}$ (equal amount to $\mathrm{CH}_{2} \mathrm{Cl}_{2}$ ) was added to the reaction solution and the $\mathrm{CH}_{2} \mathrm{Cl}_{2}$ layer was separated, dried with $\mathrm{Na}_{2} \mathrm{SO}_{4}$, filtered and concentrated in vacuo to yield a crude solid. The solid was recrystallized using the indicated solvent system to yield the pure anhydride.

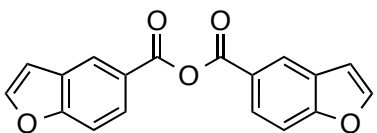

Benzofuran-5-carboxylic anhydride. The general procedure was followed using benzofuran-5-carboxylic acid (2.00 g, 12.3 mmol, 1.0 equiv), $\mathrm{EDC} \cdot \mathrm{HCl}(1.18 \mathrm{~g}, 6.15 \mathrm{mmol}, 0.50$ equiv) and $\mathrm{CH}_{2} \mathrm{Cl}_{2}(24 \mathrm{~mL})$. The title product was isolated as a white solid after recrystallization from a hot hexanes/EtOAc (10:1 ratio) solution $(1.20 \mathrm{~g}, 3.92 \mathrm{mmol}, 64 \%$ yield $){ }^{1} \mathbf{H}$ NMR $\left(400 \mathrm{MHz}, \mathrm{CDCl}_{3}\right) \delta 8.50(\mathrm{~d}, \mathrm{~J}=1.8 \mathrm{~Hz}, 2 \mathrm{H}), 8.18(\mathrm{dd}, \mathrm{J}=8.7,1.8 \mathrm{~Hz}, 2 \mathrm{H}), 7.76$ $(\mathrm{d}, \mathrm{J}=2.3 \mathrm{~Hz}, 2 \mathrm{H}), 7.63(\mathrm{~d}, \mathrm{~J}=8.7 \mathrm{~Hz}, 2 \mathrm{H}), 7.00-6.82(\mathrm{~m}, 2 \mathrm{H}) .{ }^{13} \mathbf{C}$ NMR $(101 \mathrm{MHz}$, CDCl3) $\delta 162.8,158.4,147.0,128.0,127.1,125.2,124.0,112.0,107.3$. HRMS (DART) $\mathrm{m} / \mathrm{z}$ calcd. for $\mathrm{C}_{18} \mathrm{H}_{14} \mathrm{NO}_{5}{ }^{+}\left[\mathrm{M}+\mathrm{NH}_{4}\right]^{+}: 324.3115 ; 324.0874$ found. m.p. $122-124{ }^{\circ} \mathrm{C}$. IR (thin film) 1766, 1709, 1264, 1104, 1019, $745 \mathrm{~cm}^{-1}$.

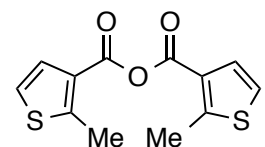

2-Methylthiophene-3-carboxylic anhydride. The general procedure was followed using 2-methylthiophene-3-carboxylic acid (2.80 g, 19.7 mmol, 1.0 equiv), $\mathrm{EDC} \cdot \mathrm{HCl}(1.89 \mathrm{~g}, 9.85 \mathrm{mmol}, 0.50$ equiv) and $\mathrm{CH}_{2} \mathrm{Cl}_{2}(40 \mathrm{~mL})$. The title product was isolated as a white solid after recrystallization from a hot hexanes solution (1.20 g, $7.09 \mathrm{mmol}, 72 \%$ yield). ${ }^{1} \mathbf{H}$ NMR $\left(400 \mathrm{MHz}, \mathrm{CDCl}_{3}\right) \delta 7.45(\mathrm{~d}, \mathrm{~J}=5.4 \mathrm{~Hz}, 2 \mathrm{H}), 7.07(\mathrm{~d}, \mathrm{~J}=5.5 \mathrm{~Hz}, 2 \mathrm{H}), 2.84(\mathrm{~d}, \mathrm{~J}=0.7$ $\mathrm{Hz}, 6 \mathrm{H}) .{ }^{13} \mathbf{C}$ NMR $\left(101 \mathrm{MHz}, \mathrm{CDCl}_{3}\right) \delta 158.7,153.8,129.3,127.0,121.8,15.9$. m.p. 90-92 ${ }^{\circ} \mathrm{C}$. IR (thin film) 3110, 3090, 1760, 1700, 1532, 1227, 997, $709 \mathrm{~cm}^{-1}$. EA Calcd. for $\mathrm{C}_{12} \mathrm{H}_{10} \mathrm{O}_{3} \mathrm{~S}_{2}$ : C, 54.12; H, 3.78. Found: C, 54.19; H, 3.75.

Alkenes: Styrene, 4-acetoxystyrene, 4-cyanostyrene and 2-methylstyrene were purchased from Alfa Aeser and used as received. 3-Vinylphenol ${ }^{6},(E)$-1-styryl-4-vinylbenzene ${ }^{7}, 1-$ tosyl-5-vinyl-1H-indole ${ }^{8}$, tert-butyl 4-(4-vinylphenyl)piperazine-1-carboxylate ${ }^{9}$ and cinnamyl methyl ether ${ }^{10}$ are known compounds and were prepared by previously reported procedures. 


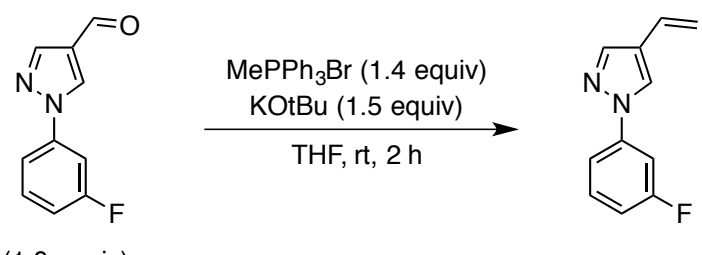

(1.0 equiv)

1-(3-Fluorophenyl)-4-vinyl-1H-pyrazole. To a $25 \mathrm{~mL}$ oven-dried round bottom flask containing a magnetic stir bar was added methyltriphenylphosphonium bromide $(1.31 \mathrm{~g}$, $3.67 \mathrm{mmol}, 1.4$ equiv) and THF (5 mL). Potassium tert-butoxide (441 mg, $3.93 \mathrm{mmol}$, 1.5 equiv) was added and the reaction mixture was stirred for $30 \mathrm{~min}$ at $\mathrm{rt}$ during which time the reaction mixture turned yellow. Then, 1-(3-fluorophenyl)-1H-pyrazole-4carbaldehyde (500 mg, $2.62 \mathrm{mmol}, 1.0$ equiv) as a solution in THF (3 mL) was added drop-wise to the reaction mixture. The reaction mixture was stirred for an additional $1.5 \mathrm{~h}$ at $\mathrm{rt}$ and then quenched with $15 \mathrm{~mL} \mathrm{H}_{2} \mathrm{O}$. The resulting mixture was extracted with EtOAc $(3 \times 10 \mathrm{~mL})$ and the combined extracts were dried with $\mathrm{Na}_{2} \mathrm{SO}_{4}$, filtered, concentrated in vacuo and purified by silica gel chromatography $\left(20 \% \mathrm{Et}_{2} \mathrm{O}\right.$ in hexanes). The title product was isolated as a colorless oil (490 mg, $2.60 \mathrm{mmol}, 99 \%$ yield). ${ }^{1} \mathbf{H}$ NMR $\left(400 \mathrm{MHz}, \mathrm{CDCl}_{3}\right) \delta 7.86(\mathrm{~d}, \mathrm{~J}=26.4 \mathrm{~Hz}, 2 \mathrm{H}), 7.54-7.34(\mathrm{~m}, 3 \mathrm{H}), 7.06-6.92$ $(\mathrm{m}, 1 \mathrm{H}), 6.60(\mathrm{dd}, \mathrm{J}=17.7,11.0 \mathrm{~Hz}, 1 \mathrm{H}), 5.62(\mathrm{~d}, \mathrm{~J}=1.2 \mathrm{~Hz}, 1 \mathrm{H}), 5.21(\mathrm{~d}, \mathrm{~J}=11.0 \mathrm{~Hz}$, $1 \mathrm{H}) .{ }^{13} \mathrm{C}$ NMR $\left(101 \mathrm{MHz}, \mathrm{CDCl}_{3}\right) \delta 164.6,162.1,141.4,141.3,139.4,130.9,130.8$, $126.19,124.2,123.4,114.1,114.1,113.7,113.3,113.1,106.8,106.5 .{ }^{19}$ F NMR (376 $\mathrm{MHz}_{\mathrm{CDCl}}$ ) $\delta$-110.9. IR (thin film) 3087, 1611, 1600, 1495, 1149, 862, 771, $677 \mathrm{~cm}^{-1}$. EA Calcd. for $\mathrm{C}_{11} \mathrm{H}_{9} \mathrm{FN}_{2}$ : C, 70.20; H, 4.82. Found: C, 70.47; H, 5.01.

\section{References}

1) Alcohol assignment: a) Lou, S.; Fu, G. C. J. Am. Chem. Soc. 2010, 132, 1264. Ketone assignment: b) Shionhara, T.; Suzuki, K. Synthesis 2003, 141. c) Lundin, P. M.; Esquivias, J.; Fu, G. C. Angew. Chem. Int. Ed. 2009, 48, 154. d) Suzuki, K.; Katayama, E.; Tsuchihashi, G. Tetrahedron Lett. 1983, 24, 4997.

2) Chung, J. Y.; Mancheno, D.; Dormer, P. G.; Variankaval, N.; Ball, R. G.; Tsou, N. N. Org. Lett. 2008, 10, 3037.

3) Funasaka, S.; Mukaiyama, T. Bull. Chem. Soc. Jpn. 2008, 81, 148.

4) Cirriez, V.; Rasson, C.; Riant, O. Adv. Synth. Catal. 2013, 355, 3137.

5) Cabré-Castellví, J.; Palomo-Coll, A.; Palomo-Coll; A. L. Synthesis 1981, 8, 616.

6) Gligorich, K. M.; Cummings, S. A.; Sigman, M. A. J. Am. Chem. Soc. 2007, 129, 14193.

7) Woodford, C. R.; Frady, E. P.; Smith, R. S.; Morey, B.; Canzi, G.; Palida, S. F.; Araneda, R. C.; Kristan Jr., W. B.; Kubiak, C. P.; Miller, E. W.; Tsien, R. Y. J. Am. Chem. Soc. 2015, 137, 1817.

8) Molander, G. A.; Brown, A. R. J. Org. Chem. 2006, 71, 9681.

9) Rakesh; Sun, D.; Lee, R. B.; Tangallapally, R. P.; Lee, R. E. Eur. J. Med. Chem. 2009, 44,460 .

10) Zhao, H.; Wang, Y.; Sha, J.; Sheng, S.; Cai, M. Tetrahedron 2008, 64, 7517. 


\section{HPLC Traces}

Note: For each entry, the top HPLC trace is a racemic sample that was prepared using standard conditions and rac-Ph-BPE ligand. For most alcohol products, the minor diastereomer can be seen in the top racemic HPLC trace. The minor diastereomer was not observed for several alcohol products where the dr was $>20: 1$.

Table 2, 4a. Hexanes/iPrOH 95/5, $1.0 \mathrm{~mL} / \mathrm{min}, 210 \mathrm{~nm}, \mathrm{AD}-\mathrm{H}$.
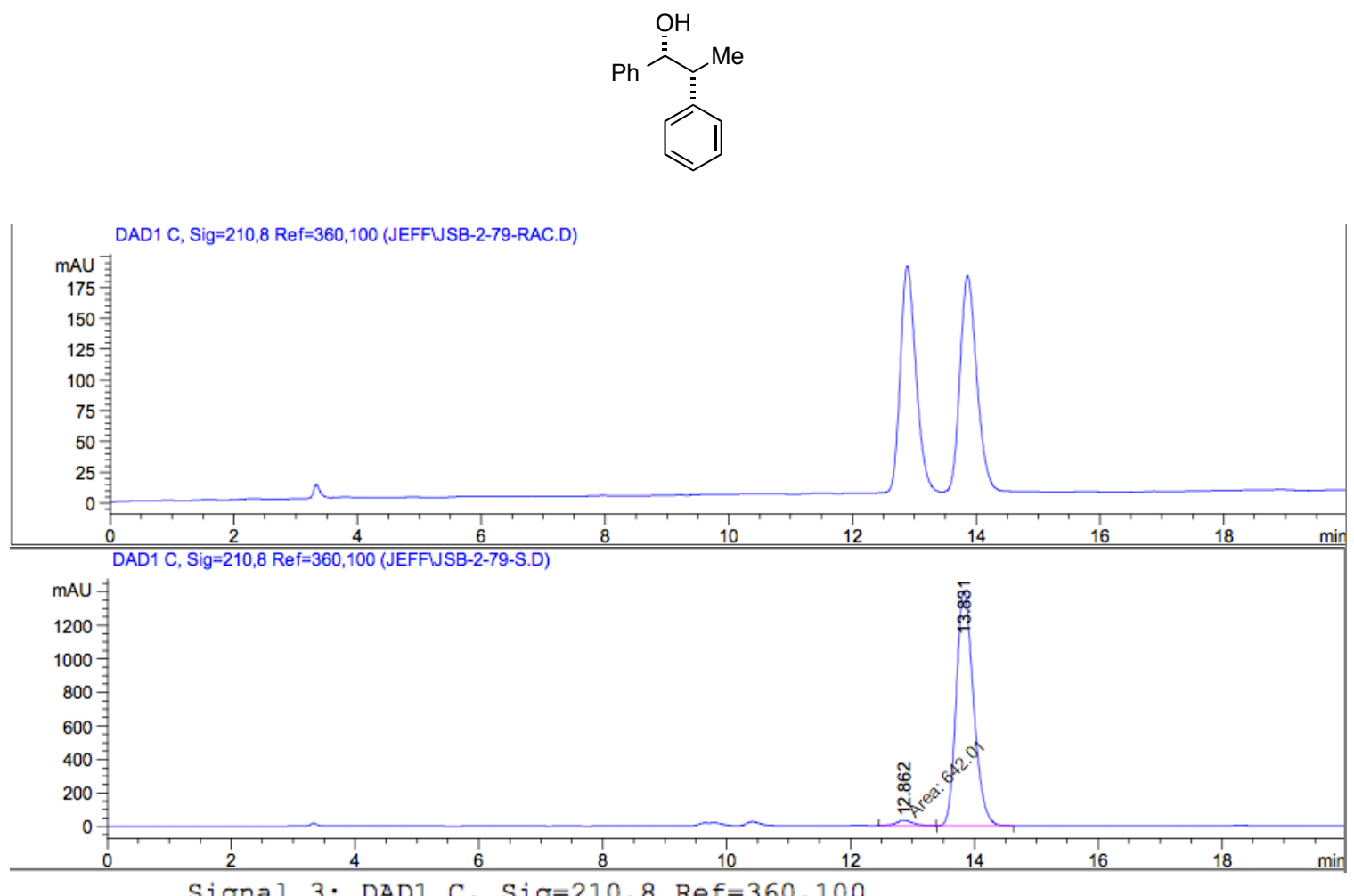

Signal 3: DAD1 C, Sig=210,8 Ref=360,100

\begin{tabular}{|c|c|c|c|c|c|c|}
\hline $\begin{array}{c}\text { Peak } \\
\#\end{array}$ & $\begin{array}{c}\text { RetTime } \\
\text { [ min] }\end{array}$ & Type & $\begin{array}{l}\text { Width } \\
\text { [ min] }\end{array}$ & $\begin{array}{c}\text { Area } \\
{\left[\mathrm{mAU}^{\star} \mathrm{s}\right]}\end{array}$ & $\begin{array}{l}\text { Height } \\
\text { [mAU] }\end{array}$ & $\begin{array}{c}\text { Area } \\
\quad \%\end{array}$ \\
\hline & & & & - & - & -- \\
\hline 1 & & $\mathbb{M} \mathrm{T}$ & 7 & 642.00952 & 6880 & 081 \\
\hline 2 & 31 & VB $R$ & 980 & $2.71733 e 4$ & 96301 & 6919 \\
\hline
\end{tabular}


Table 2, 4b. Hexanes/iPrOH 97/3, 1.0 mL/min, 230 nm, AD-H.
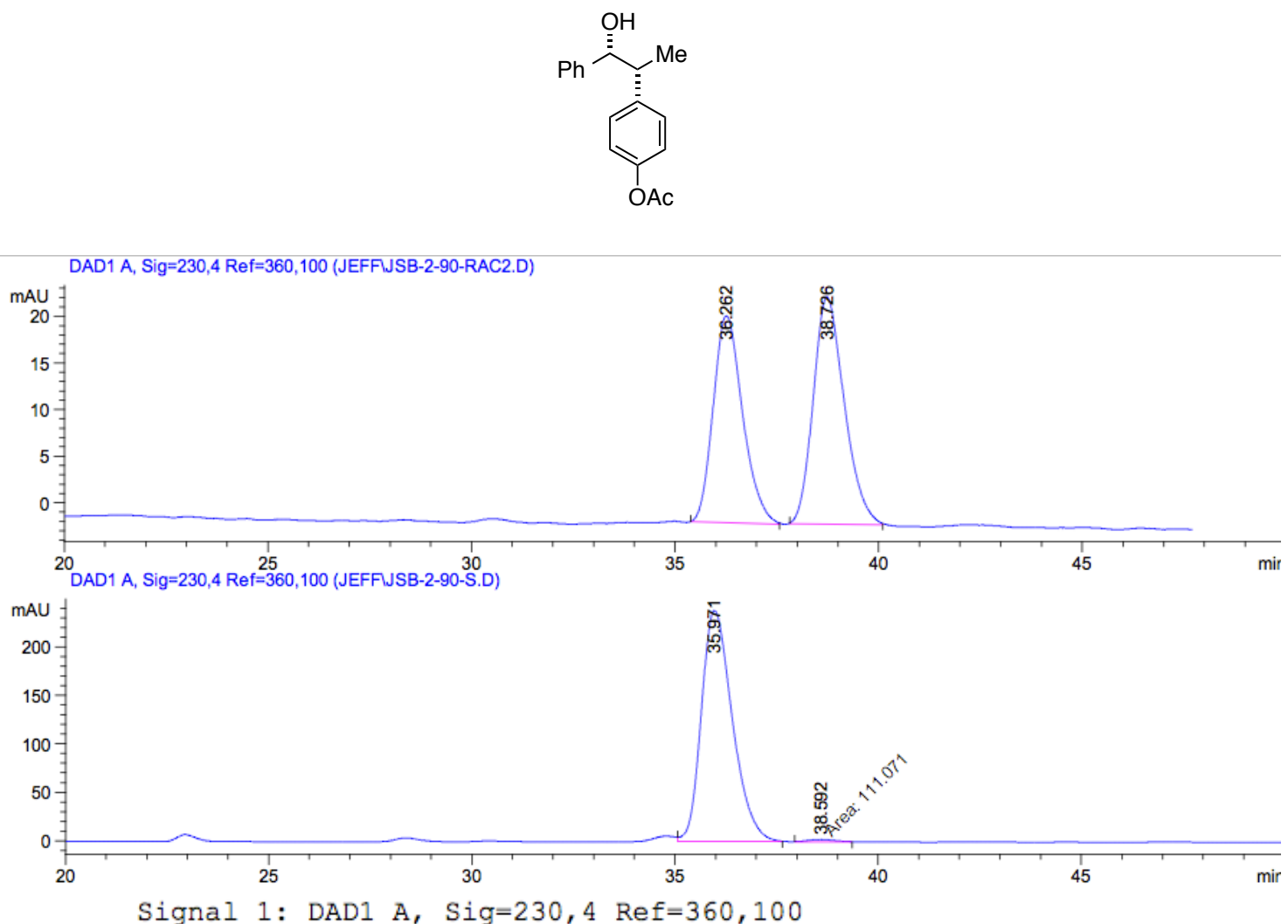

Signal 1: DAD1 A, Sig=230, 4 Ref $=360,100$

\begin{tabular}{|c|c|c|c|c|c|c|}
\hline $\begin{array}{c}\text { Peak } \\
\text { \# }\end{array}$ & $\begin{array}{l}\text { RetTime } \\
\text { [min] }\end{array}$ & Type & $\begin{array}{l}\text { Width } \\
\text { [min] }\end{array}$ & $\begin{array}{c}\text { Area } \\
{\left[\mathrm{mAU}^{\star} \mathrm{s}\right]}\end{array}$ & $\begin{array}{l}\text { Height } \\
\text { [mAU] }\end{array}$ & $\begin{array}{c}\text { Area } \\
\quad \%\end{array}$ \\
\hline & & & & -- & -- & --1 \\
\hline 1 & & & & 1.2 & 673 & 297 \\
\hline 2 & 992 & MM T & 633 & 111.07142 & 2527 & 3703 \\
\hline
\end{tabular}

Totals : $\quad 1.27630$ e4 240.85200 
Table 2, 4c. Hexanes/iPrOH 95/5, 0.8 mL/min, 230 nm, OD-H.
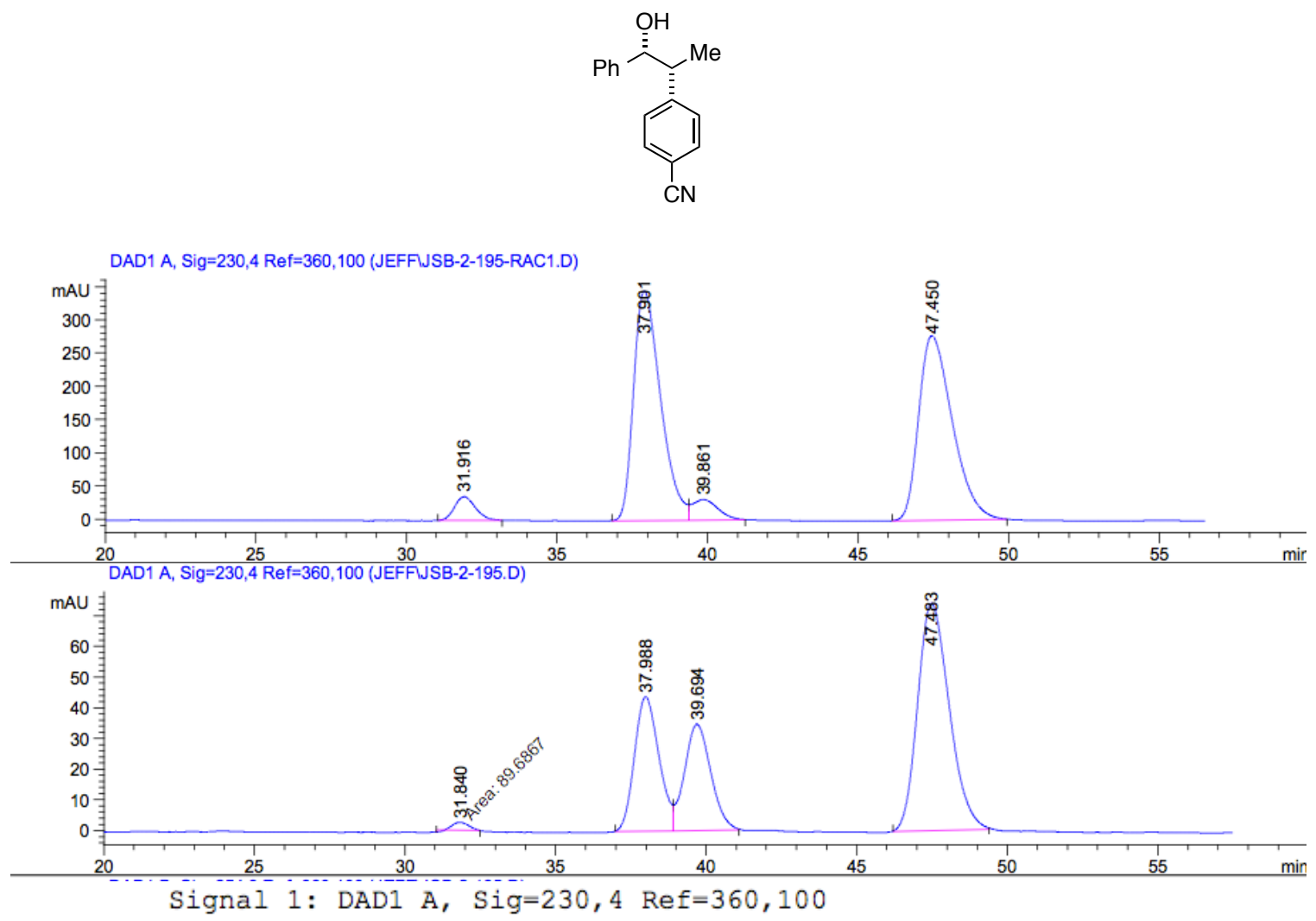

\begin{tabular}{|c|c|c|c|c|c|c|}
\hline Peak & $\begin{array}{c}\text { RetTime } \\
\text { [ min] }\end{array}$ & Type & $\begin{array}{l}\text { Width } \\
\text { [ min] }\end{array}$ & $\begin{array}{c}\text { Area } \\
{\left[\mathrm{mAU}^{*} \mathrm{~s}\right]}\end{array}$ & $\begin{array}{l}\text { Height } \\
{[\mathrm{mAU}]}\end{array}$ & $\begin{array}{c}\text { Area } \\
\frac{8}{8}\end{array}$ \\
\hline & & & & -- & ----- & ---1 \\
\hline 1 & 31.840 & MM T & 0.5860 & 89.68667 & 2.55086 & 0.8868 \\
\hline 2 & 37.988 & BV & 0.8700 & 2489.72925 & 43.87679 & 24.6189 \\
\hline 3 & 39.694 & JB & 0.9022 & 2112.82153 & 34.70666 & 20.8919 \\
\hline 4 & 47.483 & $\mathrm{BB}$ & .1226 & 5420.85303 & 74.18092 & 53.6023 \\
\hline
\end{tabular}

Totals : $\quad 1.01131 \mathrm{e} 4 \quad 155.31523$ 
Table 2, 4d. Hexanes/iPrOH 90/10, 1.0 mL/min, 230 nm, OD-H.
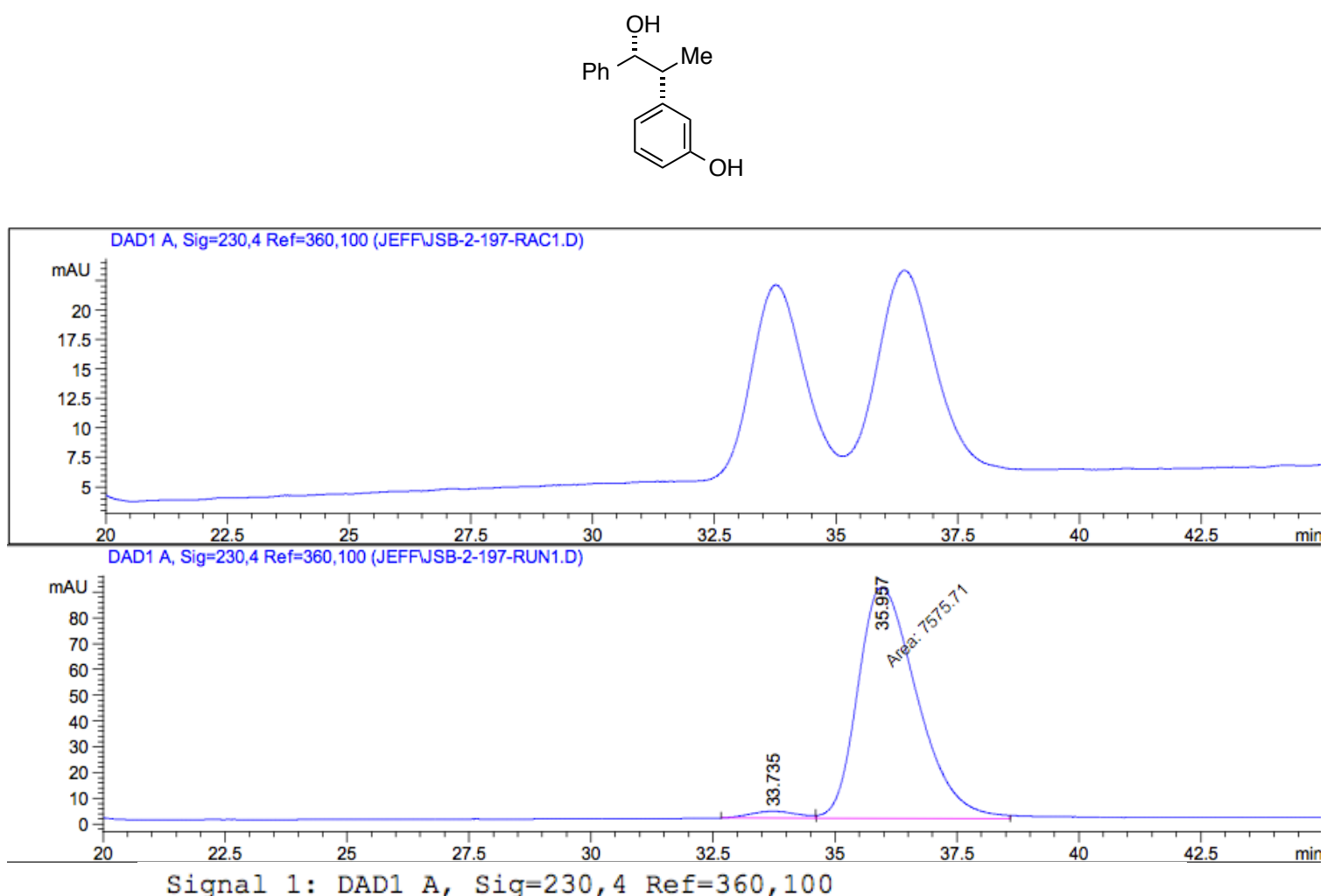

Signal 1: DAD1 A, Sig=230, 4 Ref $=360,100$

\begin{tabular}{|c|c|c|c|c|c|c|}
\hline $\begin{array}{c}\text { Peak } \\
\#\end{array}$ & $\begin{array}{l}\text { RetTime } \\
\text { [min] }\end{array}$ & Type & $\begin{array}{l}\text { Width } \\
\text { [ min] }\end{array}$ & $\begin{array}{c}\text { Area } \\
{\left[\mathrm{mAU}^{\star} \mathrm{s}\right]}\end{array}$ & $\begin{array}{l}\text { Height } \\
\text { [mAU] }\end{array}$ & $\begin{array}{c}\text { Area } \\
\quad \%\end{array}$ \\
\hline & & & & --1 & $-1-$ & \\
\hline 1 & & & 5 & 175.35834 & 603 & 24 \\
\hline 2 & 957 & $\mathrm{MT}$ & .4089 & 7575.71387 & 89.61816 & 376 \\
\hline
\end{tabular}

Totals : $\quad 7751.07220 \quad 92.22419$ 
Table 2, 4e. Hexanes/iPrOH 95/5, 1.0 mL/min, 210 nm, OD-H.
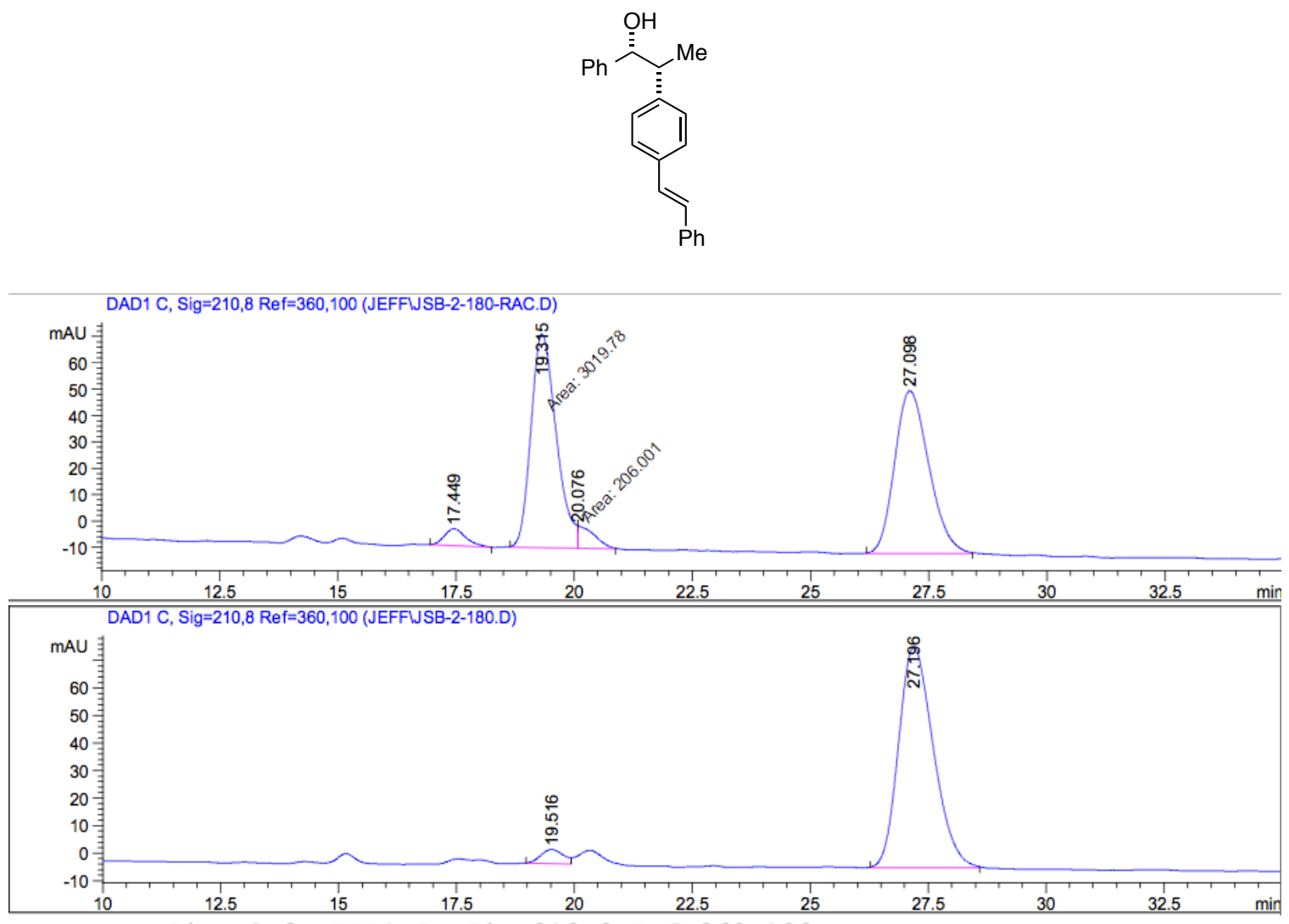

Signal 3: DAD1 C, Sig=210,8 $\operatorname{Re} f=360,100$

\begin{tabular}{|c|c|c|c|c|c|c|}
\hline $\begin{array}{c}\text { Peak } \\
\#\end{array}$ & $\begin{array}{l}\text { RetTime } \\
\text { [min] }\end{array}$ & Type & $\begin{array}{l}\text { width } \\
\text { [min] }\end{array}$ & $\begin{array}{c}\text { Area } \\
{\left[\mathrm{mAU}^{\star} \mathrm{s}\right]}\end{array}$ & $\begin{array}{l}\text { Height } \\
\text { [mAU] }\end{array}$ & $\begin{array}{c}\text { Area } \\
\quad 8\end{array}$ \\
\hline \multicolumn{7}{|c|}{ 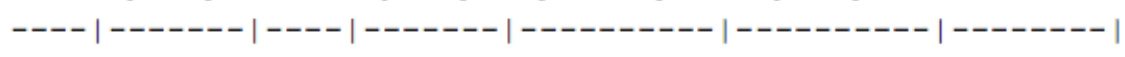 } \\
\hline 1 & 19.516 & BV & 0.4912 & 173.55191 & 5.17642 & 4.0496 \\
\hline 2 & 27.196 & $\mathrm{BB}$ & 0.7961 & 4112.11768 & 80.20988 & 95.9504 \\
\hline 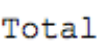 & : & & & 4285.66959 & 38631 & \\
\hline
\end{tabular}


Table 2, 4f. Hexanes/iPrOH 80/20, 1.0 mL/min, 210 nm, AD-H.
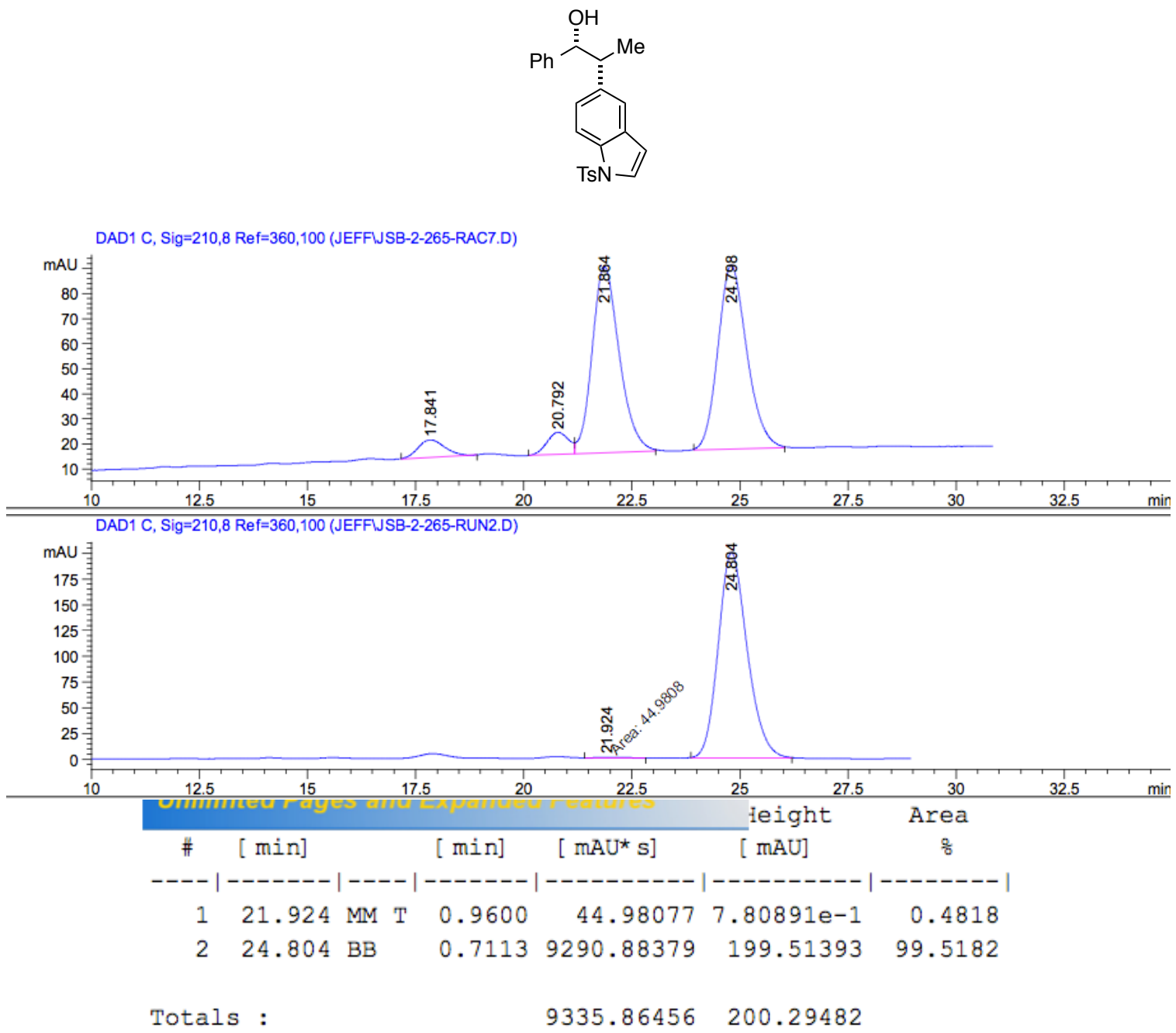
Table 2, 4g. Hexanes/iPrOH 95/5, 1.0 mL/min, 210 nm, OD-H.
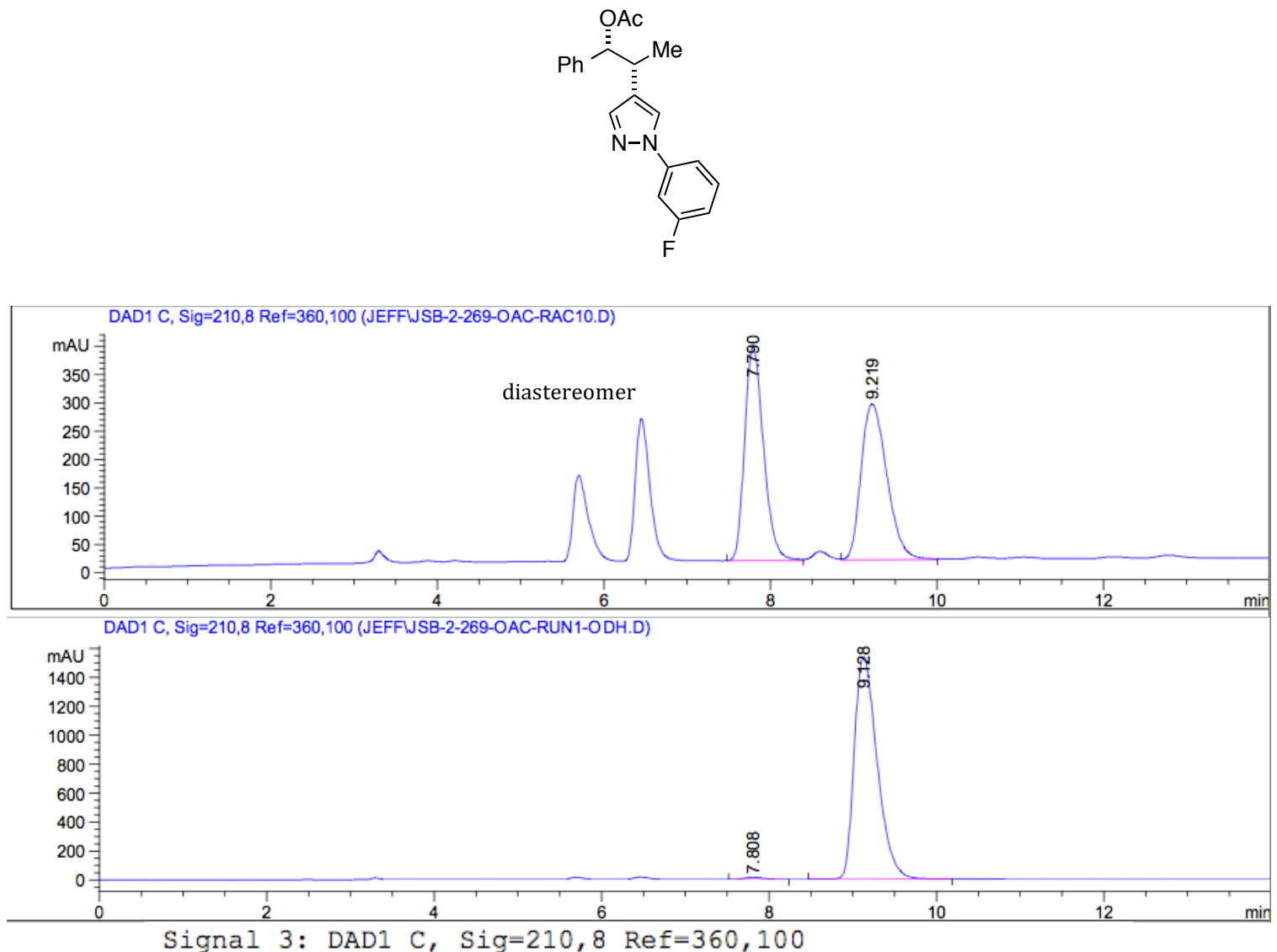

\begin{tabular}{|c|c|c|c|c|c|c|}
\hline $\begin{array}{c}\text { Peak } \\
\#\end{array}$ & $\begin{array}{c}\text { RetTime } \\
\text { [min] }\end{array}$ & Type & $\begin{array}{l}\text { Width } \\
\text { [ } \mathrm{min}]\end{array}$ & $\begin{array}{c}\text { Area } \\
{\left[\mathrm{mAU}^{\star} \mathrm{s}\right]}\end{array}$ & $\begin{array}{l}\text { Height } \\
\text { [mAU] }\end{array}$ & $\begin{array}{c}\text { Area } \\
\quad 8\end{array}$ \\
\hline \multicolumn{7}{|c|}{$----|-------|----|-------|----------|----------|--------$} \\
\hline 1 & 7.808 & $\mathrm{BB}$ & 0.2359 & 238.29890 & 15.62817 & 0.8067 \\
\hline 2 & 9.128 & $\mathrm{BB}$ & 0.2943 & $2.93016 \mathrm{e} 4$ & 1537.46265 & 99.1933 \\
\hline otal & & & & $2.95399 e 4$ & 9082 & \\
\hline
\end{tabular}


Table 2, 4h. Hexanes/iPrOH 90/10, 1.0 mL/min, 254 nm, AD-H.
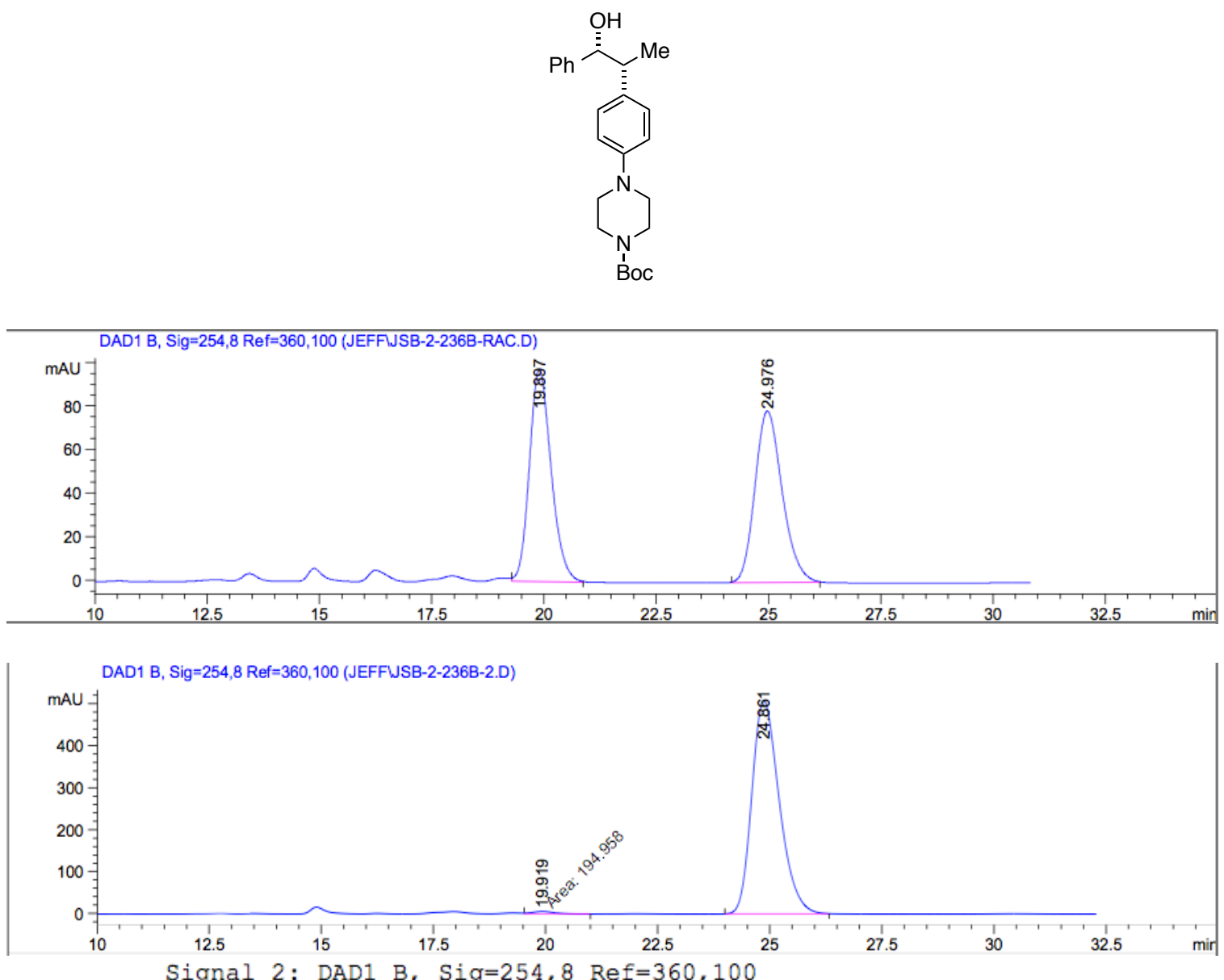

Signal 2: DAD1 B, Sig=254, 8 Ref $=360,100$

\begin{tabular}{|c|c|c|c|c|c|c|}
\hline $\begin{array}{c}\text { Peak } \\
\#\end{array}$ & $\begin{array}{c}\text { RetTime } \\
\text { [min] }\end{array}$ & Type & $\begin{array}{l}\text { Width } \\
\text { [min] }\end{array}$ & $\begin{array}{c}\text { Area } \\
{\left[\mathrm{mAU}^{\star} \mathrm{s}\right]}\end{array}$ & $\begin{array}{l}\text { Height } \\
\text { [mAU] }\end{array}$ & $\begin{array}{c}\text { Area } \\
\%\end{array}$ \\
\hline \multicolumn{7}{|c|}{$----|-------|----|-------|----------|----------|--------\mid$} \\
\hline 1 & 19.919 & FM & 0.5580 & 194.95776 & 5.82327 & 0.8929 \\
\hline 2 & 24.861 & $\mathrm{BB}$ & 0.6546 & $2.16392 \mathrm{e} 4$ & 508.37949 & 99.1071 \\
\hline that & s : & & & $2.18341 \mathrm{e} 4$ & 514.20275 & \\
\hline
\end{tabular}


Table 2, 4i. Hexanes/iPrOH 95/5, 1.0 mL/min, 230 nm, AD-H.
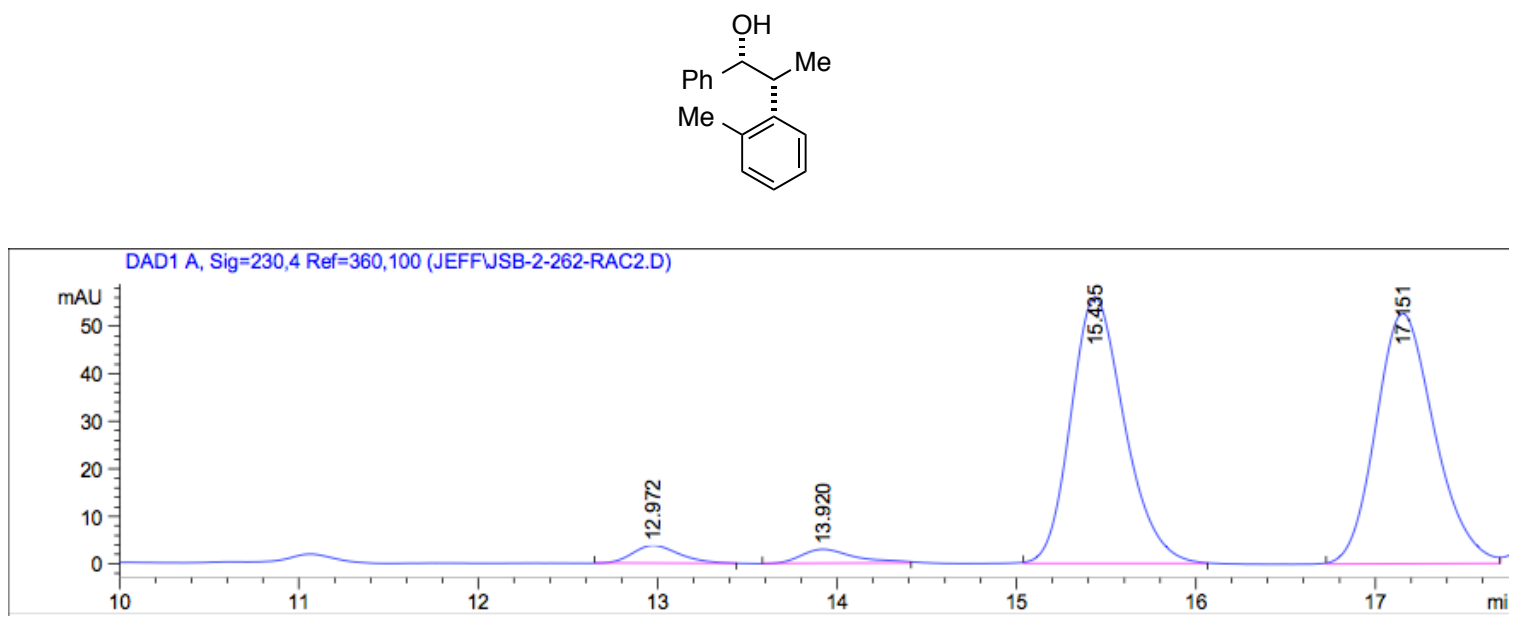
DAD1 A, Sig=230,4 Ref=360,100 (JEFFWSB-2-262-RUN1.D)

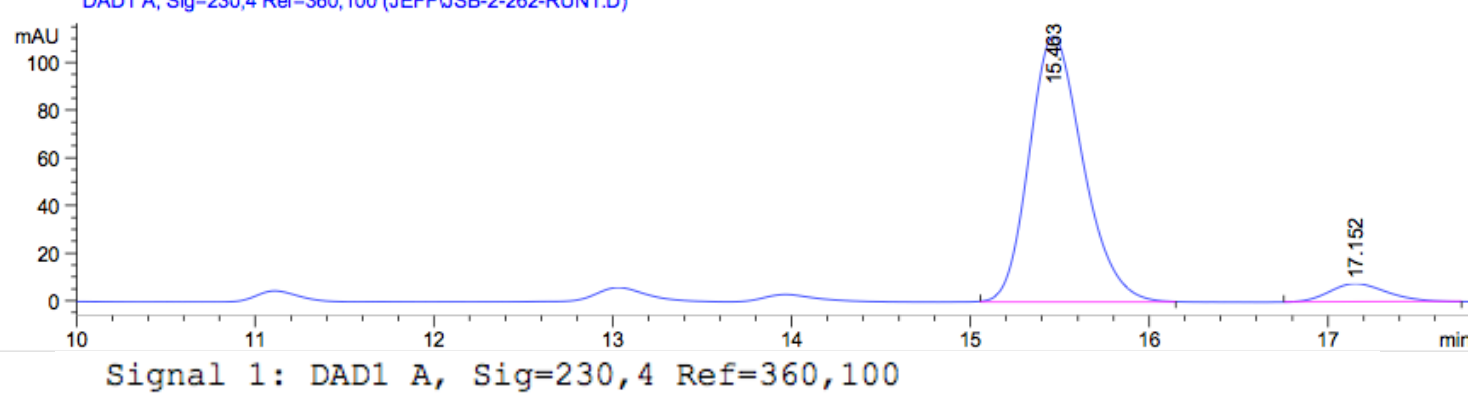

Signal 1: DAD1 A, Sig=230,4 Ref $=360,100$

Peak RetTime Type width Area Height Area

\# $[\mathrm{min}] \quad[\mathrm{min}]\left[\mathrm{MAU}^{*} \mathrm{~s}\right] \quad[\mathrm{mAU}] \quad \%$

----|--------|-----|-------|-----------|----------|---------

$\begin{array}{lllllll}1 & 15.463 & \text { BB } & 0.3223 & 2334.27197 & 111.49459 & 93.1676\end{array}$

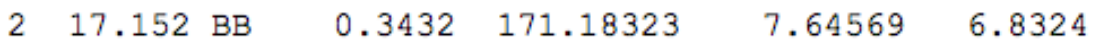

Totals : $\quad 2505.45520119 .14029$ 
Table 2, 4j. Hexanes/iPrOH 95/5, 1.0 mL/min, 210 nm, OD-H.
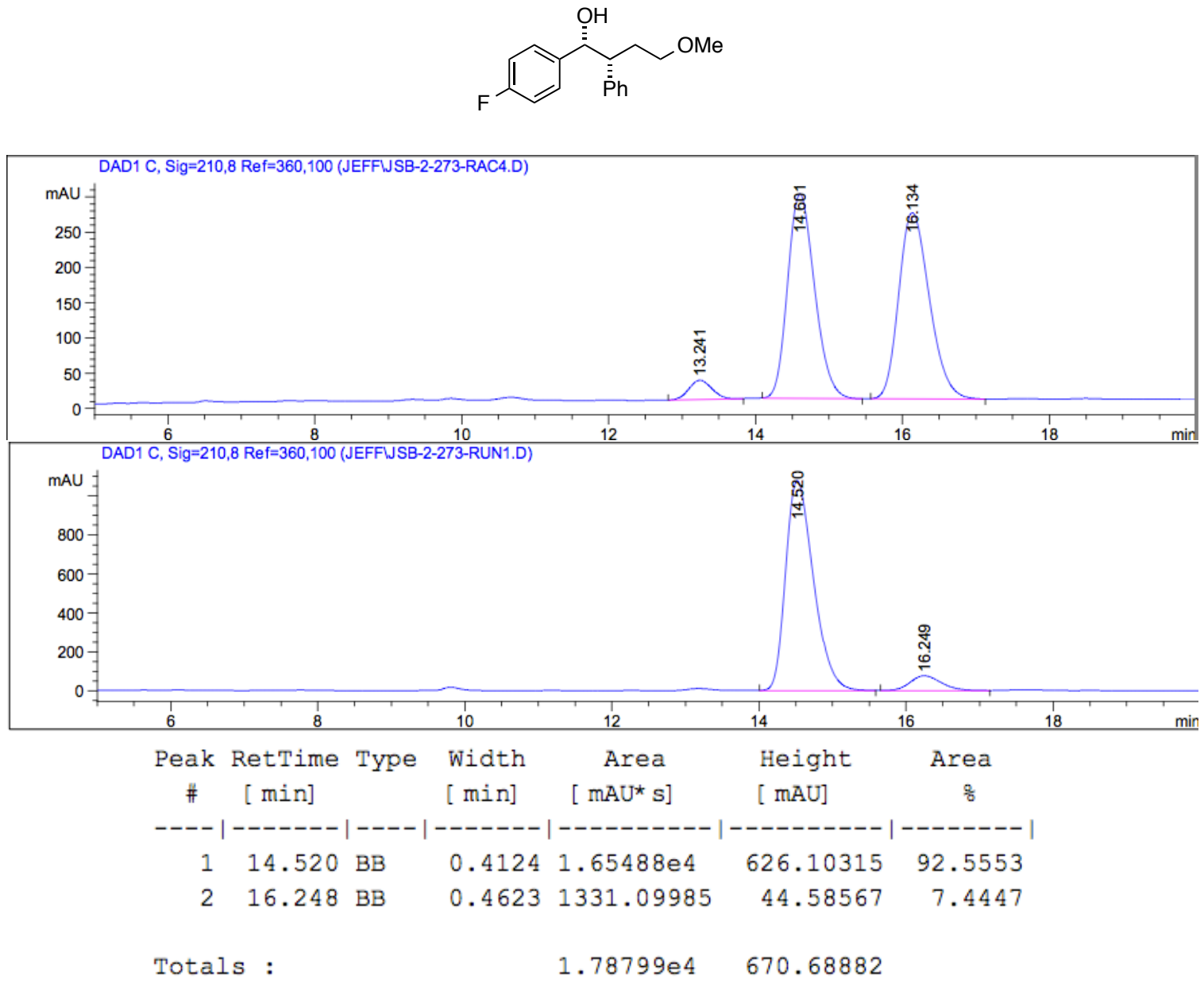
Table 2, 4k. Hexanes/iPrOH 95/5, 1.0 mL/min, 210 nm, AD-H.
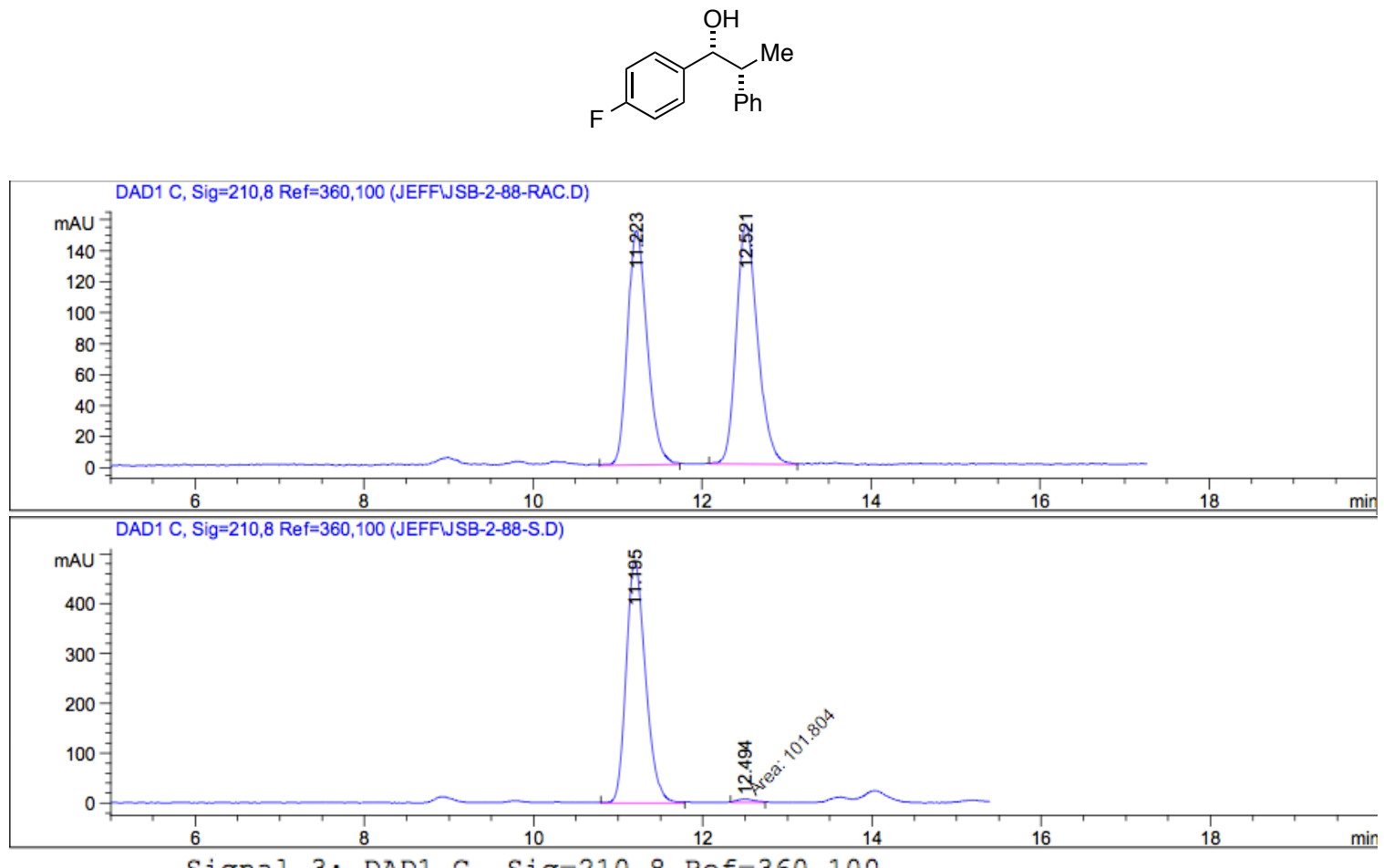

Signal 3: DAD1 C, Sig=210, 8 Ref $=360,100$

\begin{tabular}{|c|c|c|c|c|c|c|}
\hline $\begin{array}{c}\text { Peak } \\
\#\end{array}$ & $\begin{array}{c}\text { RetTime } \\
\text { [min] }\end{array}$ & Type & $\begin{array}{l}\text { width } \\
\text { [min] }\end{array}$ & $\begin{array}{c}\text { Area } \\
{\left[\mathrm{mAU}^{\star} \mathrm{s}\right]}\end{array}$ & $\begin{array}{l}\text { Height } \\
\text { [ mAU] }\end{array}$ & $\begin{array}{c}\text { Area } \\
\quad \%\end{array}$ \\
\hline \multicolumn{7}{|c|}{$----|-------|----|-------|----------|----------|--------\mid$} \\
\hline 1 & 11.195 & VB & 0.2470 & 7795.59619 & 486.24942 & 98.7109 \\
\hline 2 & 12.494 & MM T & 0.2476 & 101.80399 & 6.85372 & 1.2891 \\
\hline ota & & & & 7897.40018 & 0314 & \\
\hline
\end{tabular}


Table 2, 4l. Hexanes/iPrOH 95/5, 1.0 mL/min, 230 nm, AD-H.
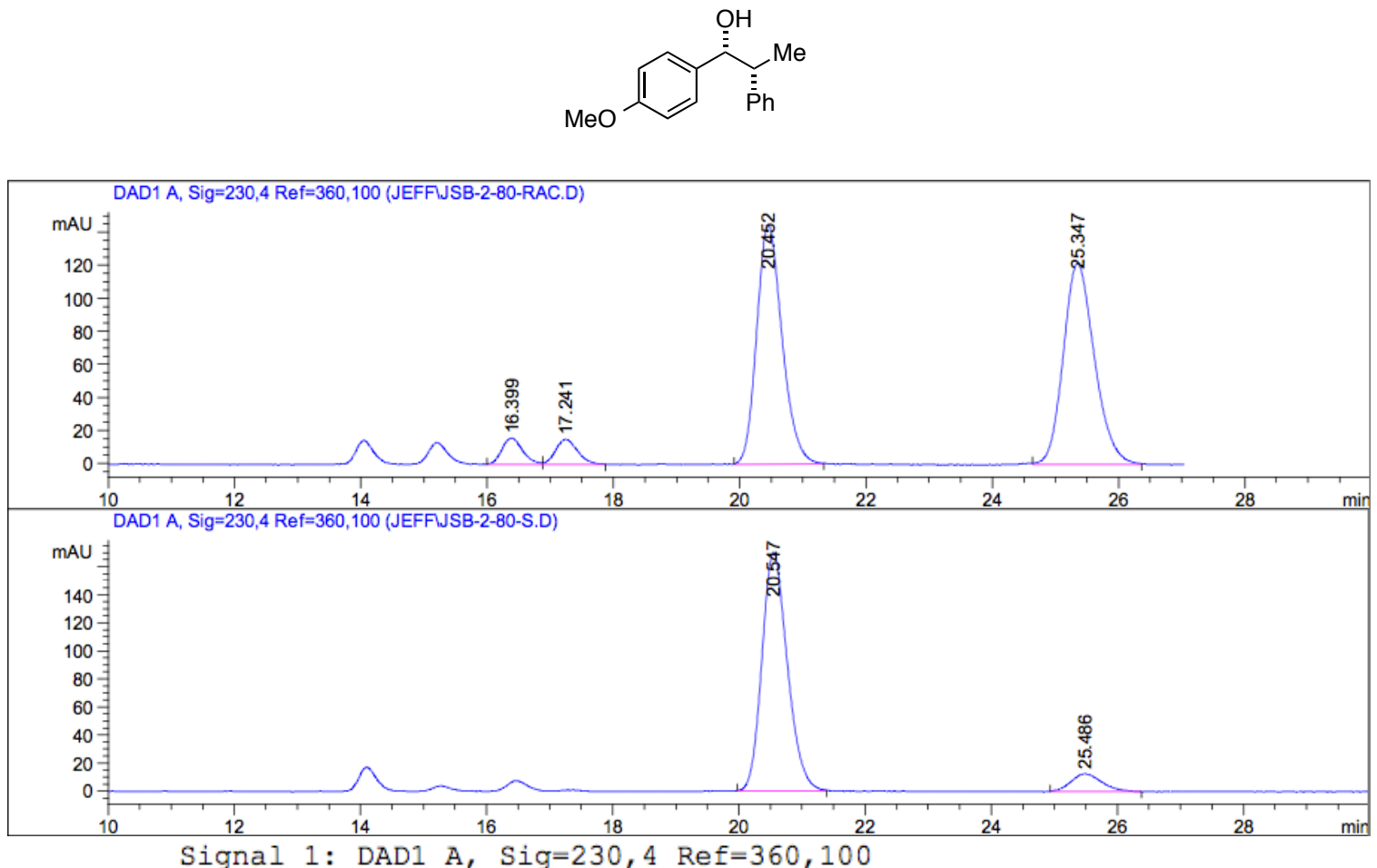

\begin{tabular}{|c|c|c|c|c|c|c|}
\hline $\begin{array}{c}\text { Peak } \\
\#\end{array}$ & $\begin{array}{c}\text { RetTime } \\
\text { [min] }\end{array}$ & Type & $\begin{array}{l}\text { Width } \\
\text { [min] }\end{array}$ & $\begin{array}{c}\text { Area } \\
{\left[\mathrm{mAU}^{\star} \mathrm{s}\right]}\end{array}$ & $\begin{array}{l}\text { Height } \\
\text { [mAU] }\end{array}$ & $\begin{array}{c}\text { Area } \\
\quad \frac{8}{\partial}\end{array}$ \\
\hline & & & & -- & --- & ---1 \\
\hline 1 & & $B B$ & & 4772.63818 & 953 & 127 \\
\hline 0 & 86 & $\mathrm{BB}$ & 148 & 436.94229 & 12.71967 & 3873 \\
\hline
\end{tabular}

Totals:

$5209.58047 \quad 182.90919$ 
Table 2, 4m. Hexanes/iPrOH 97/3, 1.0 mL/min, 210 nm, AD-H.
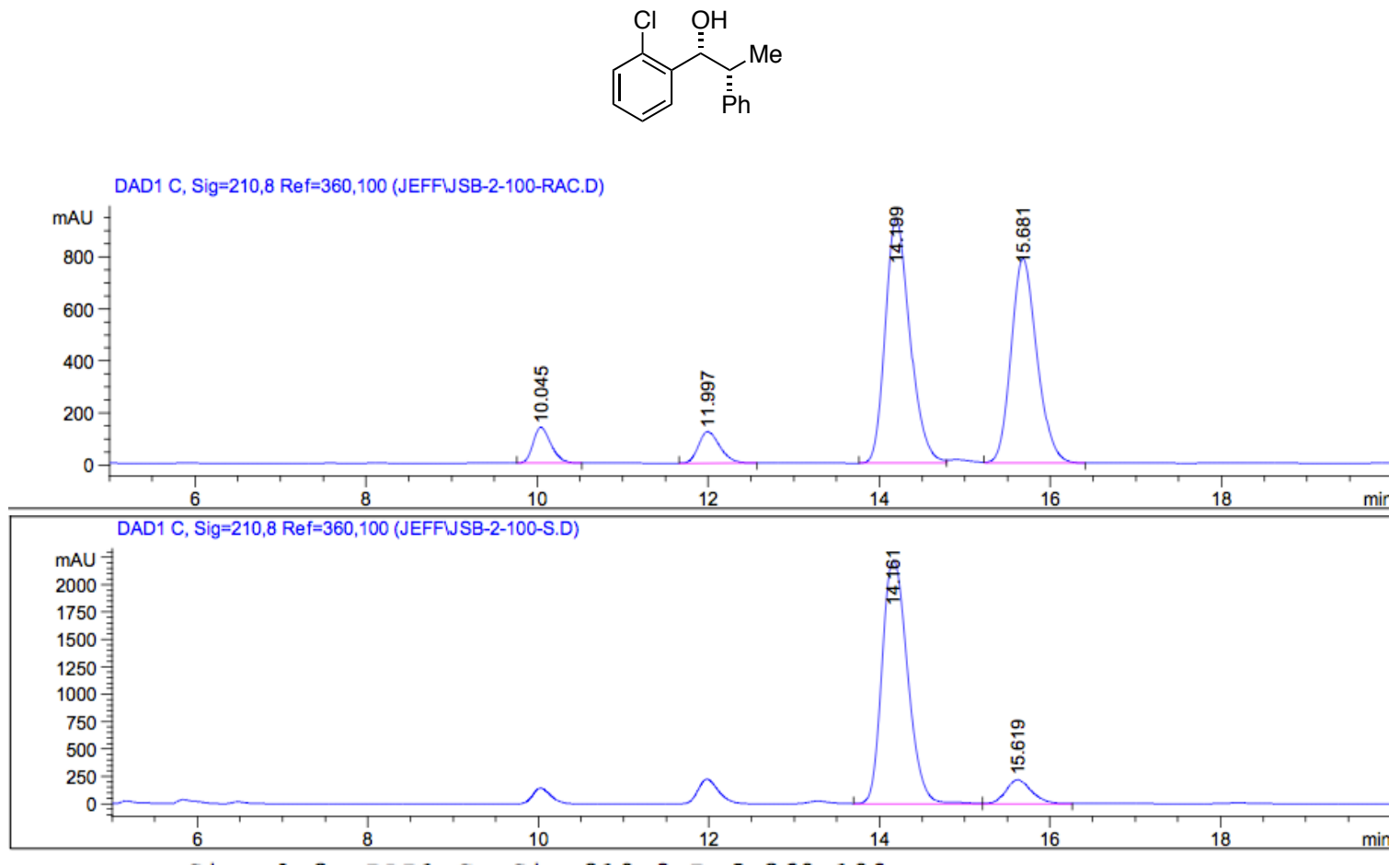

Signal 3: DAD1 C, Sig $=210,8$ Ref $=360,100$

\begin{tabular}{|c|c|c|c|c|c|c|}
\hline $\begin{array}{c}\text { Peak } \\
\#\end{array}$ & $\begin{array}{c}\text { RetTime } \\
\text { [min] }\end{array}$ & Type & $\begin{array}{l}\text { Width } \\
\text { [min] }\end{array}$ & $\begin{array}{c}\text { Area } \\
{\left[\mathrm{mAU}^{\star} \mathrm{s}\right]}\end{array}$ & $\begin{array}{l}\text { Height } \\
\text { [ } \mathrm{mAU}]\end{array}$ & $\begin{array}{c}\text { Area } \\
\quad \%\end{array}$ \\
\hline \multicolumn{7}{|c|}{ 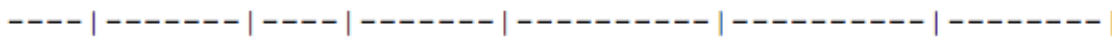 } \\
\hline 1 & 14.161 & VV & 0.3297 & $4.67660 e 4$ & 2220.88647 & 90.9717 \\
\hline 2 & 15.619 & VV & 0.3258 & 4641.21338 & 218.48190 & 9.0283 \\
\hline נהלט & & & & $5.14072 \mathrm{e} 4$ & 6838 & \\
\hline
\end{tabular}


Table 2, 4n. Hexanes/iPrOH 97/3, 1.0 mL/min, 210 nm, AD-H.

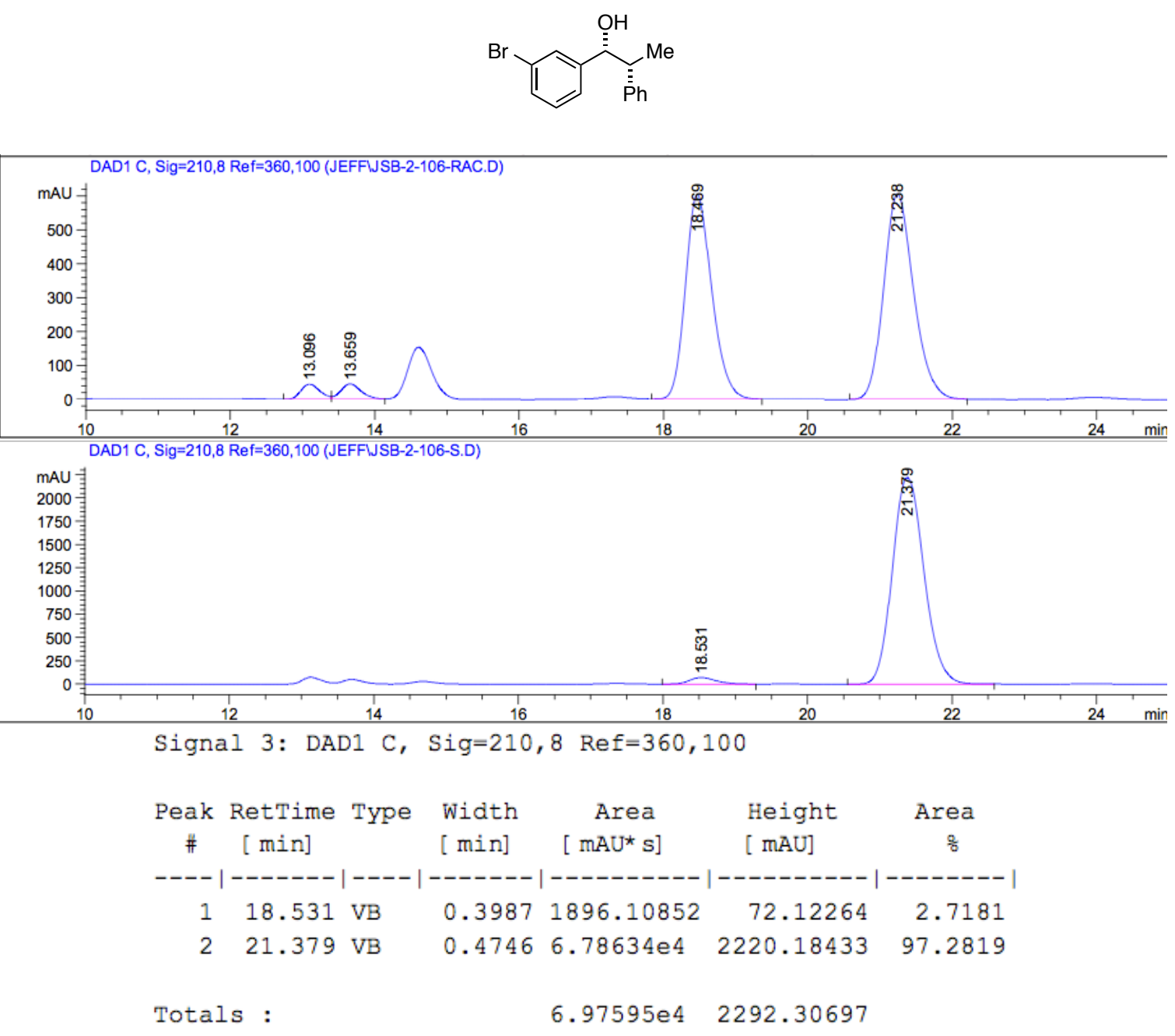


Table 2, 4o. Hexanes/iPrOH 98/2, 0.8 mL/min, 210 nm, AD-H.
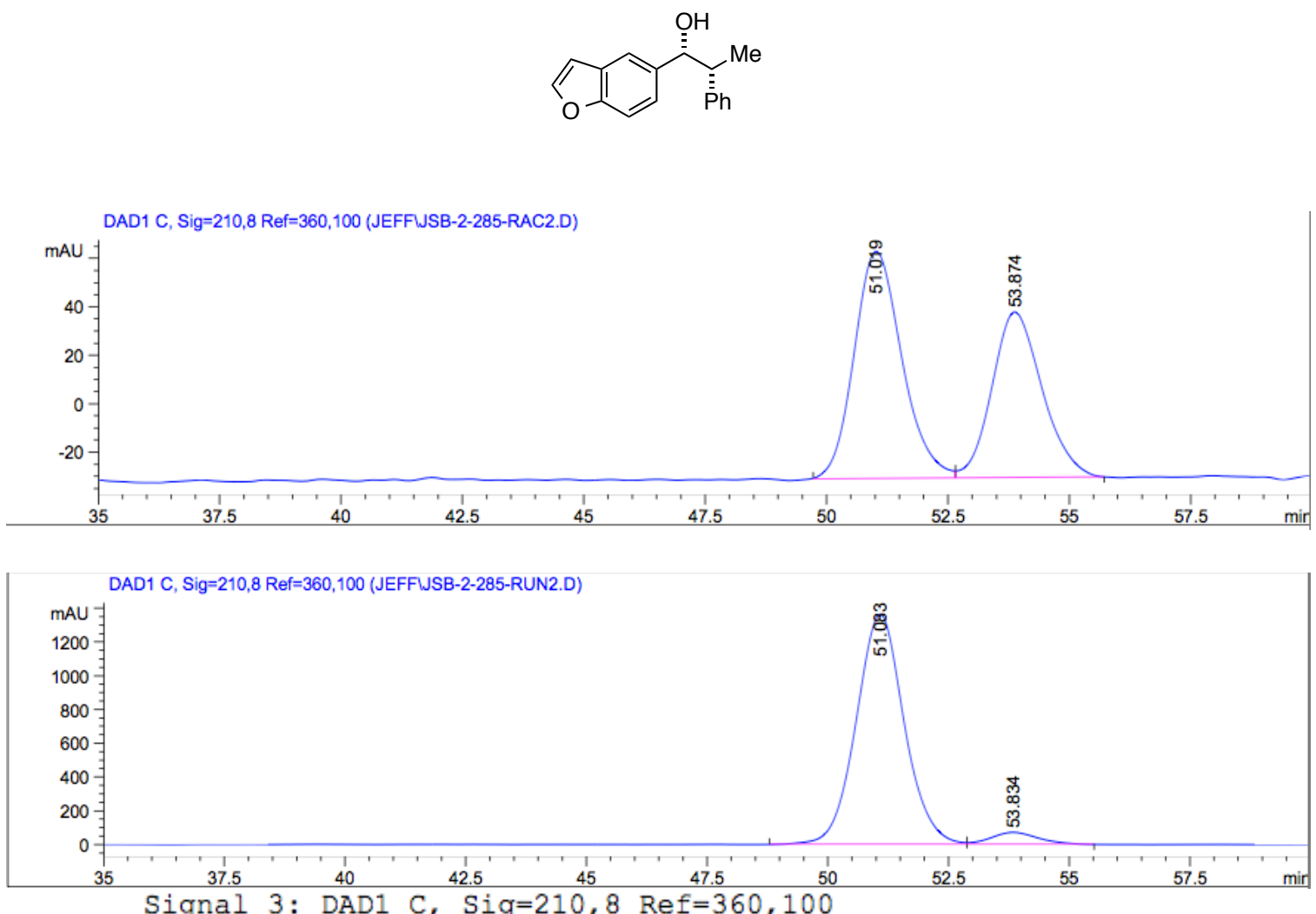

Signal 3: DAD1 C, Sig=210,8 Ref=360,100

\begin{tabular}{|c|c|c|c|c|c|c|}
\hline $\begin{array}{c}\text { Peak } \\
\#\end{array}$ & $\begin{array}{c}\text { RetTime } \\
\text { [min] }\end{array}$ & Type & $\begin{array}{l}\text { Width } \\
\text { [min] }\end{array}$ & $\begin{array}{c}\text { Area } \\
{\left[\mathrm{mAU}^{\star} \mathrm{s}\right]}\end{array}$ & $\begin{array}{l}\text { Height } \\
\text { [mAU] }\end{array}$ & $\begin{array}{c}\text { Area } \\
\%\end{array}$ \\
\hline & & & & | --------- & | --------- & ------- \\
\hline 1 & 51.083 & BV & 1.0584 & $9.45140 \mathrm{e} 4$ & 1364.02795 & 95.0371 \\
\hline 2 & 53.834 & VB & 1.0447 & 4935.61523 & 71.73275 & 4.9629 \\
\hline
\end{tabular}

Totals : $\quad 9.94496 \mathrm{e} 4 \quad 1435.76070$ 
Table 2, 4p. Hexanes/iPrOH 95/5, 1.0 mL/min, 210 nm, OD-H.
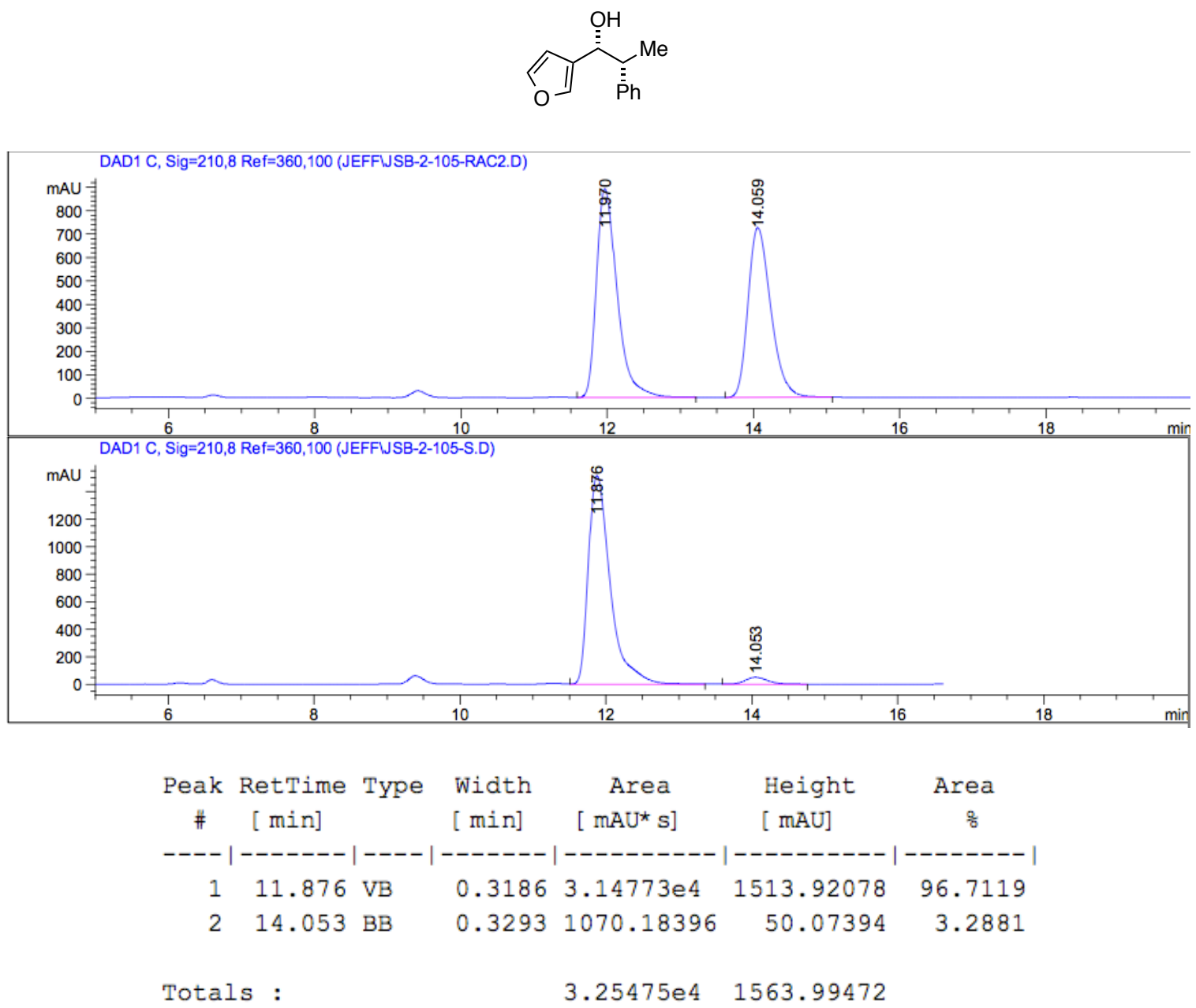
Table 2, 4q. Hexanes/iPrOH 97/3, 1.0 mL/min, 210 nm, AD-H.

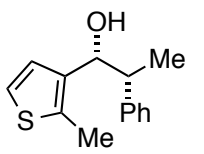

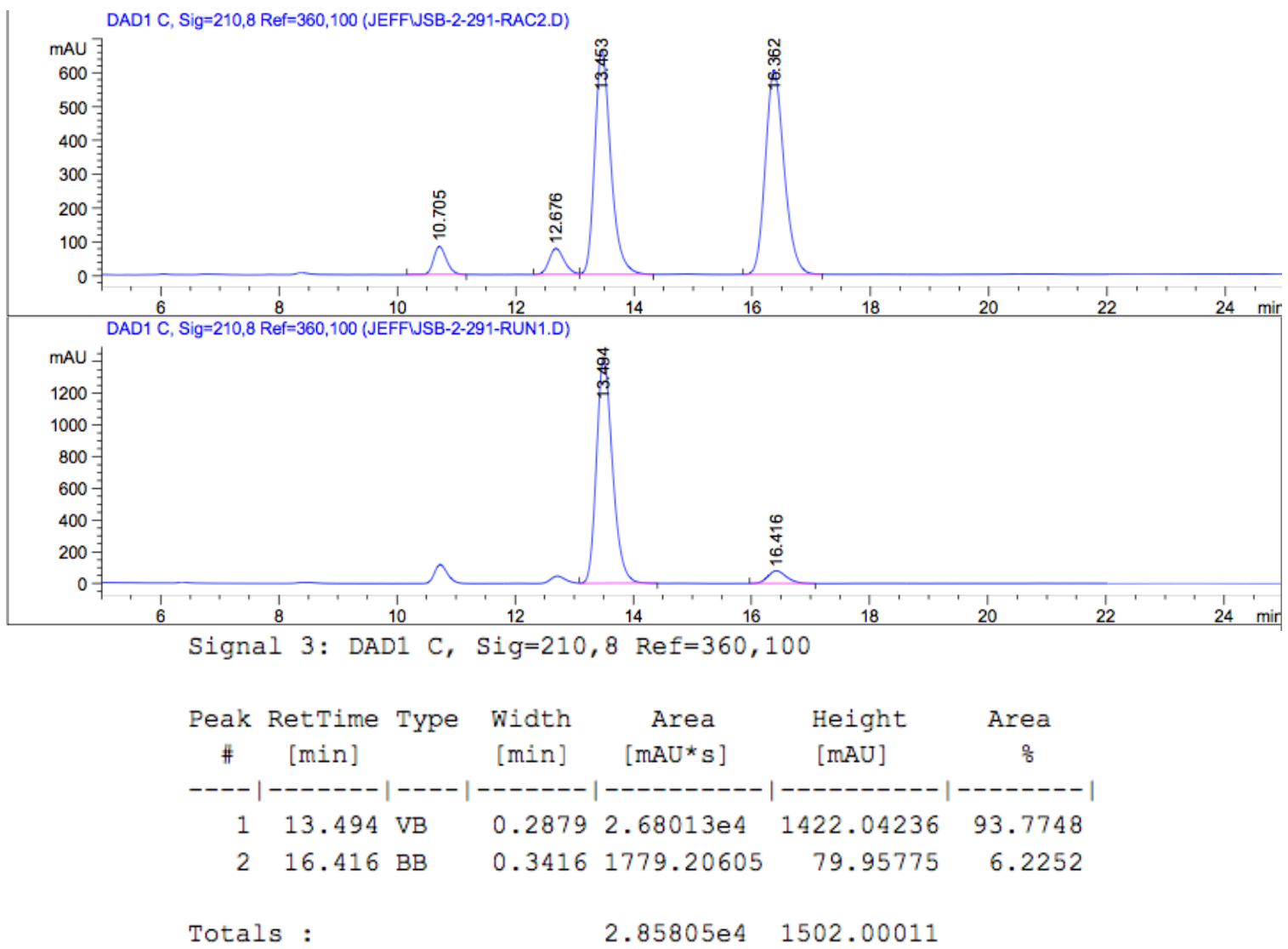


Scheme 2a, 3a. Hexanes/iPrOH 98/2, 0.8 mL/min, 254 nm, AD-H.<smiles>CC(C(=O)c1ccccc1)c1ccccc1</smiles>
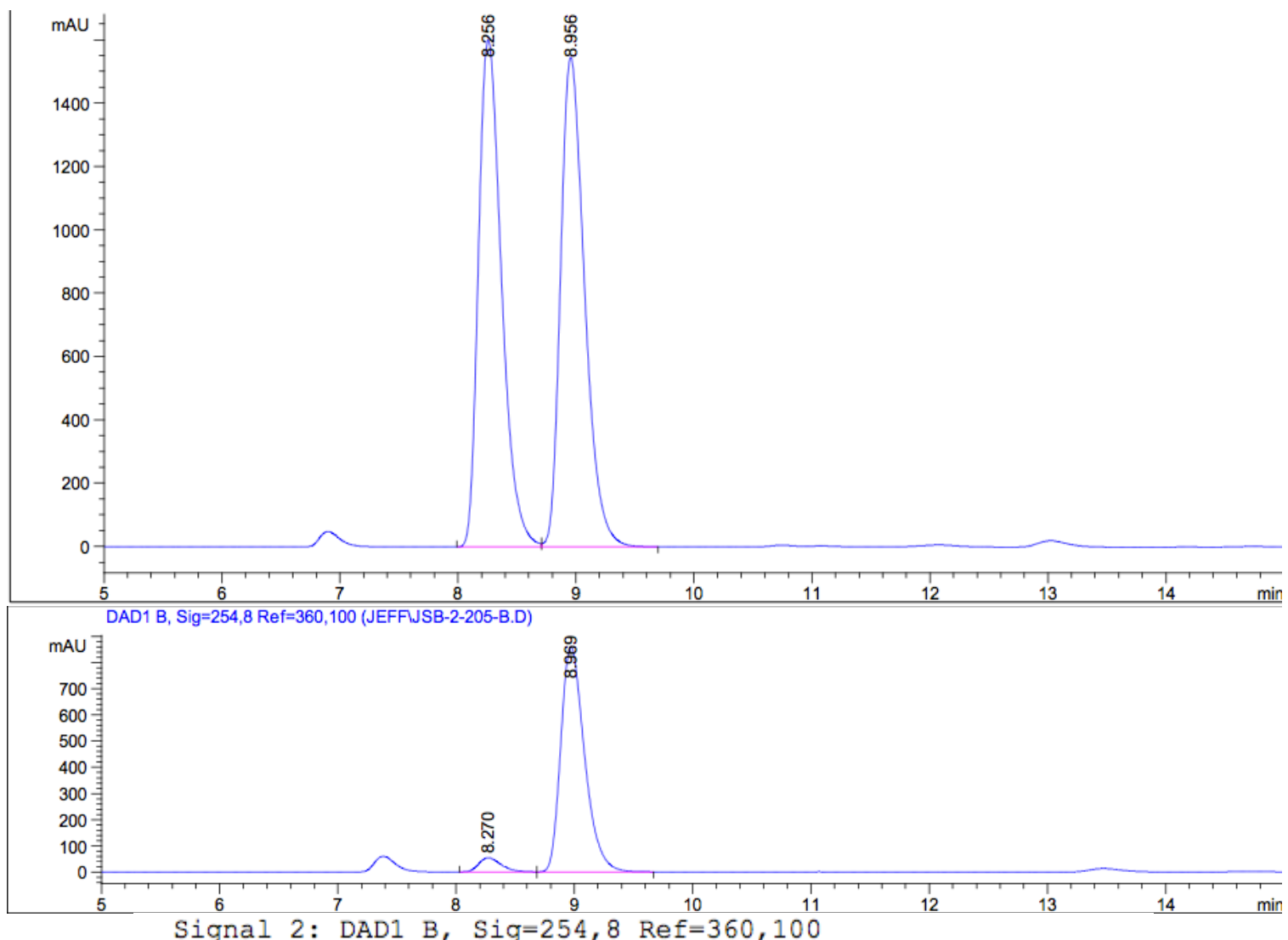

\begin{tabular}{|c|c|c|c|c|c|c|}
\hline $\begin{array}{c}\text { Peak } \\
\#\end{array}$ & $\begin{array}{l}\text { RetTime } \\
\text { [ min] }\end{array}$ & Type & $\begin{array}{l}\text { Width } \\
\text { [ min] }\end{array}$ & $\begin{array}{c}\text { Area } \\
{\left[\mathrm{mAU}^{\star} \mathrm{s}\right]}\end{array}$ & $\begin{array}{l}\text { Height } \\
{[\mathrm{mAU}]}\end{array}$ & $\begin{array}{c}\text { Area } \\
\frac{\circ}{6}\end{array}$ \\
\hline-1 & - & & -- & -------- & | & ----- \\
\hline 1 & 8.270 & BV & 0.2047 & 717.81818 & 54.14338 & 5.6262 \\
\hline 2 & 8.969 & VB & 0.2149 & $1.20406 \mathrm{e} 4$ & 862.31183 & 94.3738 \\
\hline ta. & & & & $1.27584 \mathrm{e} 4$ & 916.45520 & \\
\hline
\end{tabular}


Scheme 2a, 3b. Hexanes/iPrOH 96/4, 1.0 mL/min, 210 nm, AD-H.
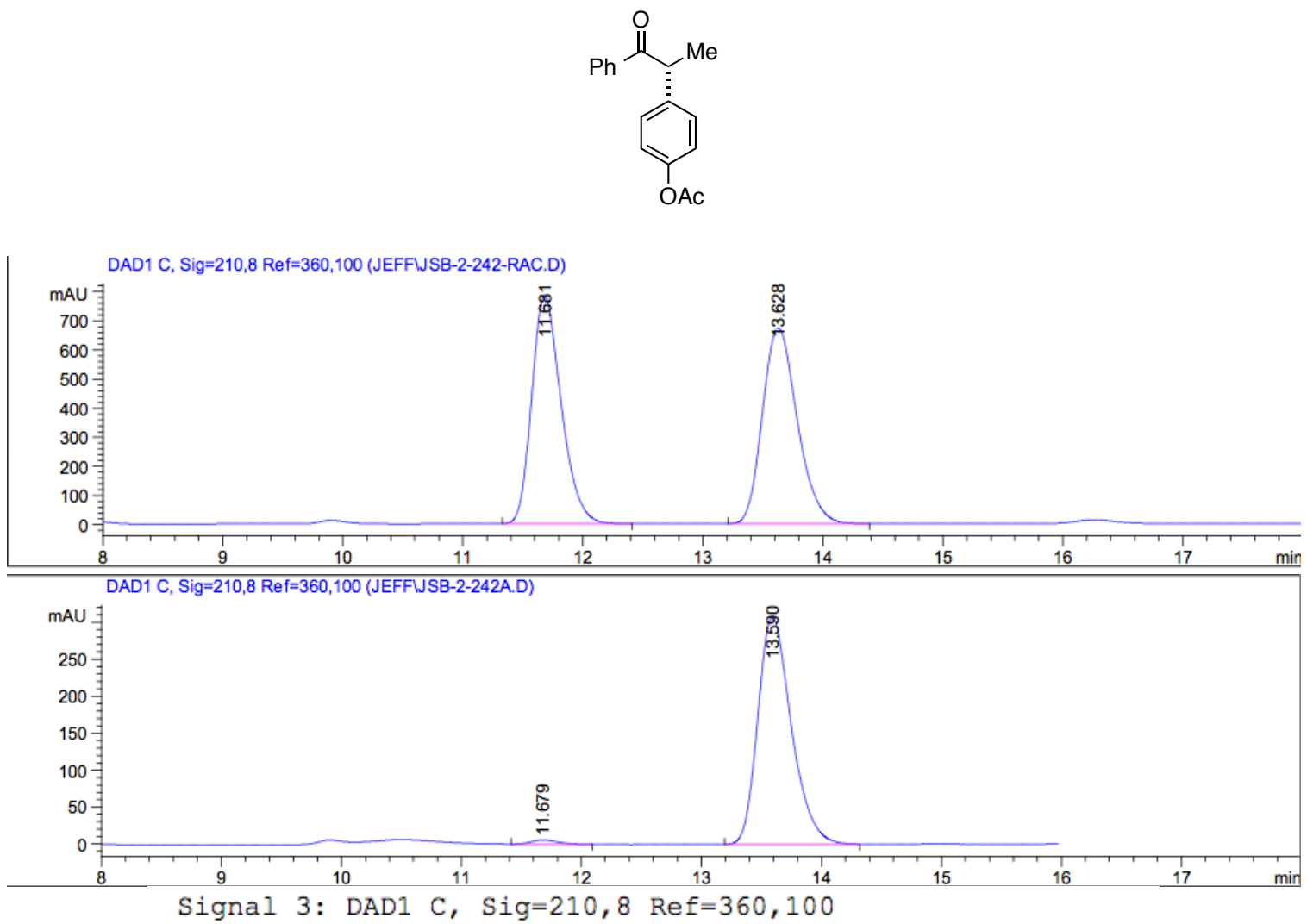

\begin{tabular}{|c|c|c|c|c|c|c|}
\hline $\begin{array}{c}\text { Peak } \\
\#\end{array}$ & $\begin{array}{c}\text { RetTime } \\
\text { [min] }\end{array}$ & Type & $\begin{array}{l}\text { Width } \\
\text { [min] }\end{array}$ & $\begin{array}{c}\text { Area } \\
{\left[\mathrm{mAU}^{\star} \mathrm{s}\right]}\end{array}$ & $\begin{array}{l}\text { Height } \\
\text { [ mAU] }\end{array}$ & $\begin{array}{c}\text { Area } \\
\quad \%\end{array}$ \\
\hline \multicolumn{7}{|c|}{ 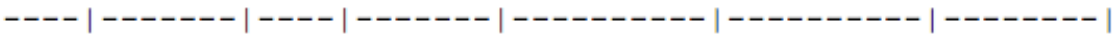 } \\
\hline 1 & 11.679 & VB & 0.2568 & 103.52910 & 6.07669 & 1.7029 \\
\hline 2 & 13.590 & $\mathrm{BB}$ & 0.2981 & 5976.14160 & 308.41000 & 98.2971 \\
\hline Tota] & : & & & 6079.67070 & 314.48670 & \\
\hline
\end{tabular}


Scheme 2a, 3c. Hexanes/iPrOH 95/5, 1.0 mL/min, 230 nm, OD-H.<smiles>C[C@H](C(=O)c1ccc(F)cc1)c1ccccc1</smiles>
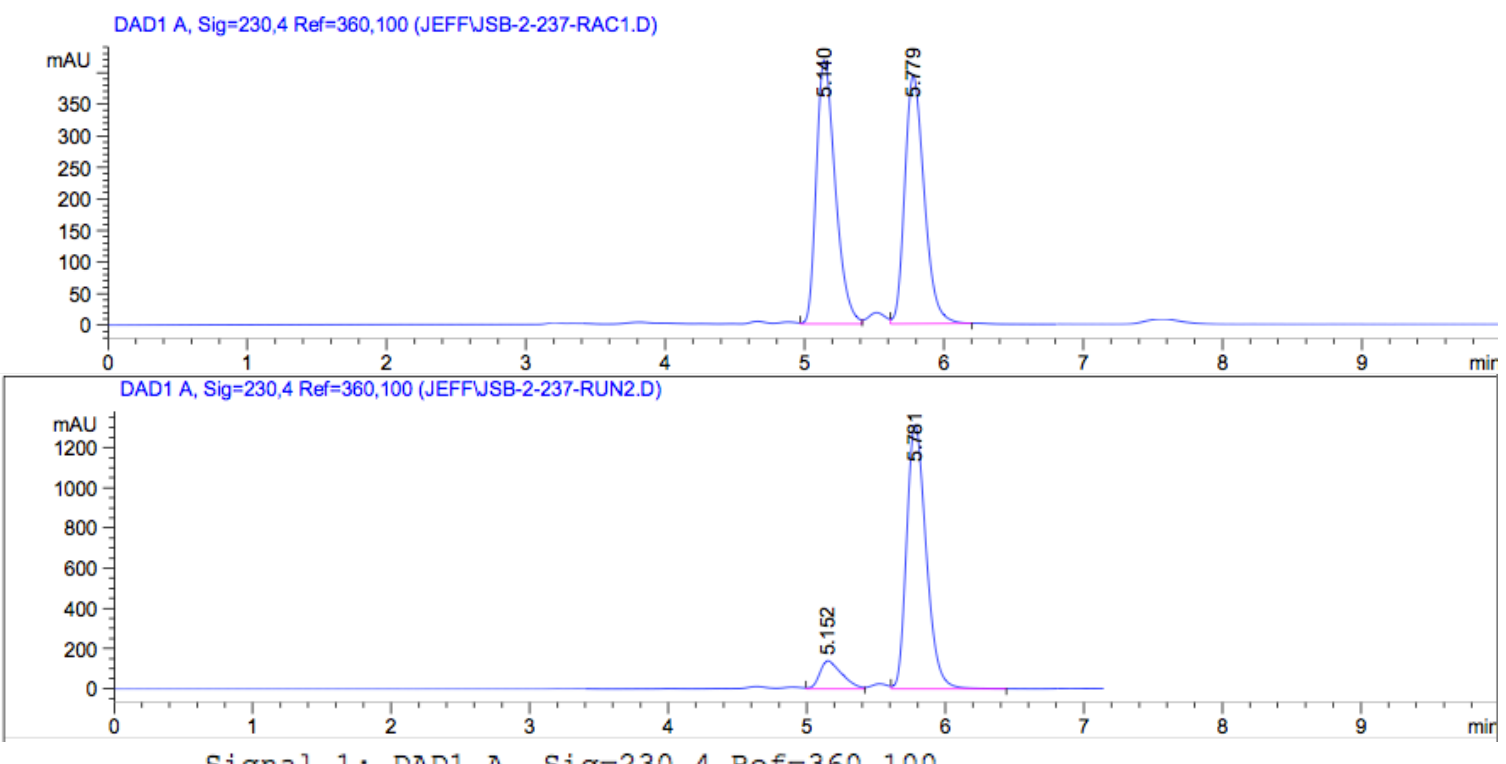

Signal 1: DAD1 A, Sig=230, 4 Ref $=360,100$

\begin{tabular}{|c|c|c|c|c|c|c|}
\hline $\begin{array}{l}\text { eak } \\
\text { \# }\end{array}$ & $\begin{array}{c}\text { RetTime } \\
\text { [min] }\end{array}$ & ype & $\begin{array}{l}\text { Width } \\
\text { [min] }\end{array}$ & $\begin{array}{c}\text { Area } \\
{\left[\mathrm{mAU}^{\star} \mathrm{s}\right]}\end{array}$ & $\begin{array}{l}\text { Height } \\
{[\mathrm{mAU}]}\end{array}$ & $\begin{array}{c}\text { Area } \\
\qquad \frac{\circ}{6}\end{array}$ \\
\hline 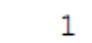 & & & & & & \\
\hline & & & 2 & $1.28814 \mathrm{e} 4$ & 1315.67871 & 89.3302 \\
\hline
\end{tabular}

Totals : $\quad 1.44200 \mathrm{e} 4 \quad 1454.85231$ 
Scheme 2a, 3d. Hexanes/iPrOH 96/4, 1.0 mL/min, 210 nm, AD-H.<smiles>CC(=O)c1ccoc1</smiles>
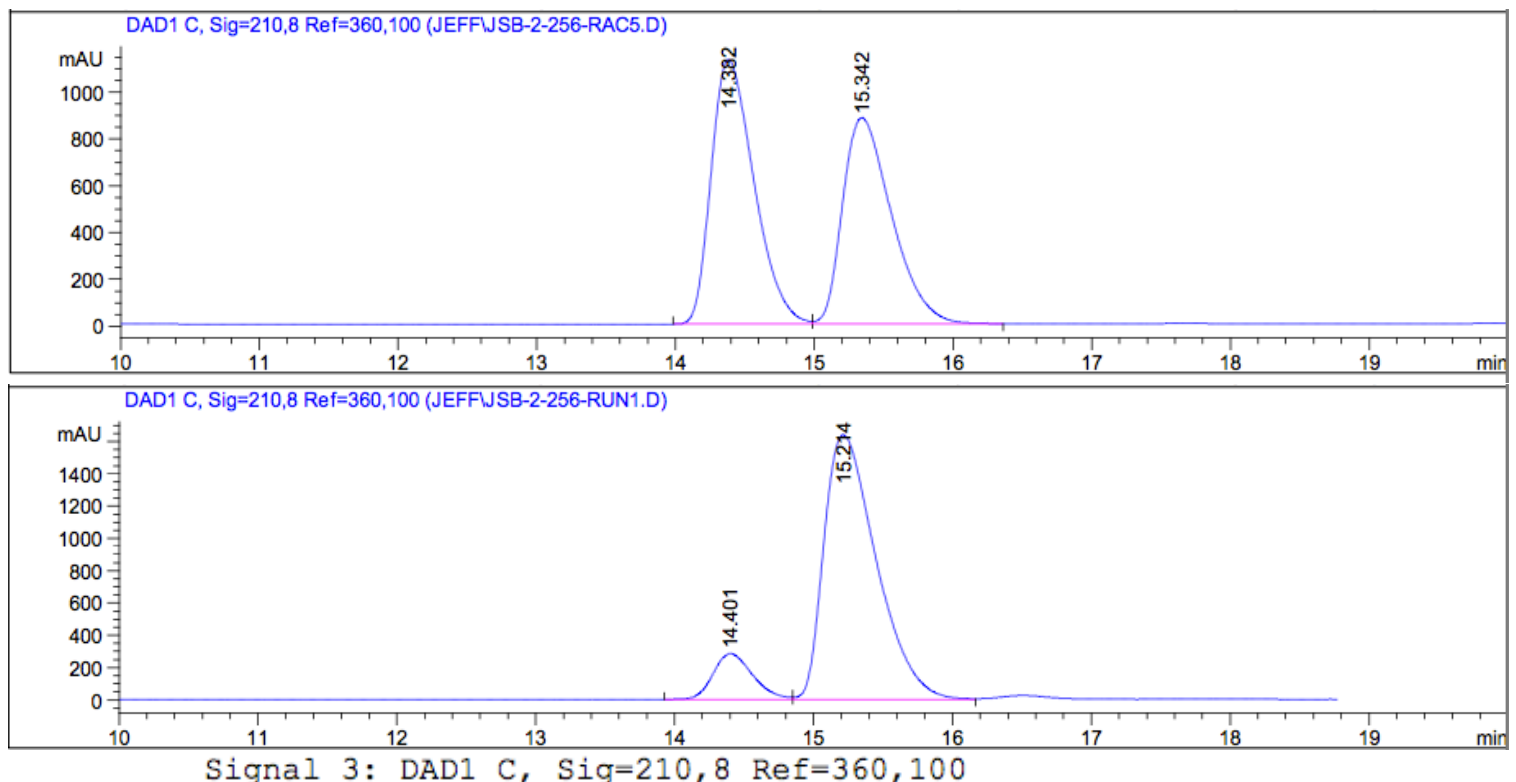

Signal 3: DAD1 C, Sig=210, 8 Ref $=360,100$

\begin{tabular}{|c|c|c|c|c|c|c|}
\hline $\begin{array}{c}\text { Peak } \\
\#\end{array}$ & $\begin{array}{c}\text { RetTime } \\
\text { [min] }\end{array}$ & Type & $\begin{array}{l}\text { Width } \\
\text { [min] }\end{array}$ & $\begin{array}{c}\text { Area } \\
{\left[\mathrm{mAU}^{\star} \mathrm{s}\right]}\end{array}$ & $\begin{array}{l}\text { Height } \\
\text { [mAU] }\end{array}$ & $\begin{array}{c}\text { Area } \\
\quad \%\end{array}$ \\
\hline \multicolumn{7}{|c|}{$----|-------|----|-------|----------|----------|--------\mid$} \\
\hline 1 & 14.401 & BV & 0.3075 & 5607.68408 & 282.55939 & 11.5882 \\
\hline 2 & 15.214 & VV & 0.4027 & $4.27835 e 4$ & 1638.38025 & 88.4118 \\
\hline & & & & $4.83912 \mathrm{e} 4$ & 93964 & \\
\hline
\end{tabular}


Scheme 1b, Reduction of Ketone 3a with $(S, S)$-Ph-BPE. Hexanes/iPrOH 99/1, 1.0 $\mathrm{mL} / \mathrm{min}, 210 \mathrm{~nm}, \mathrm{OD}-\mathrm{H}$.

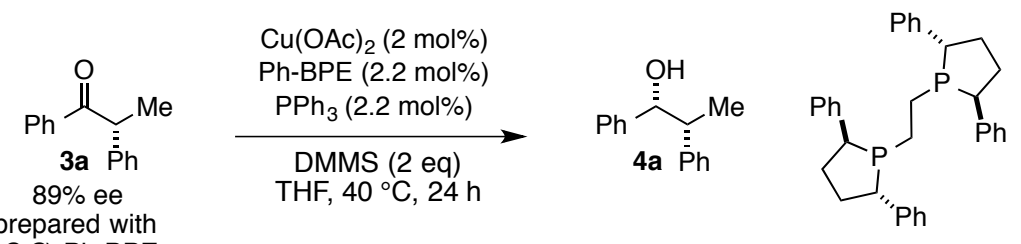

$(S, S)$-Ph-BPE

$(S, S)-P h-B P E$

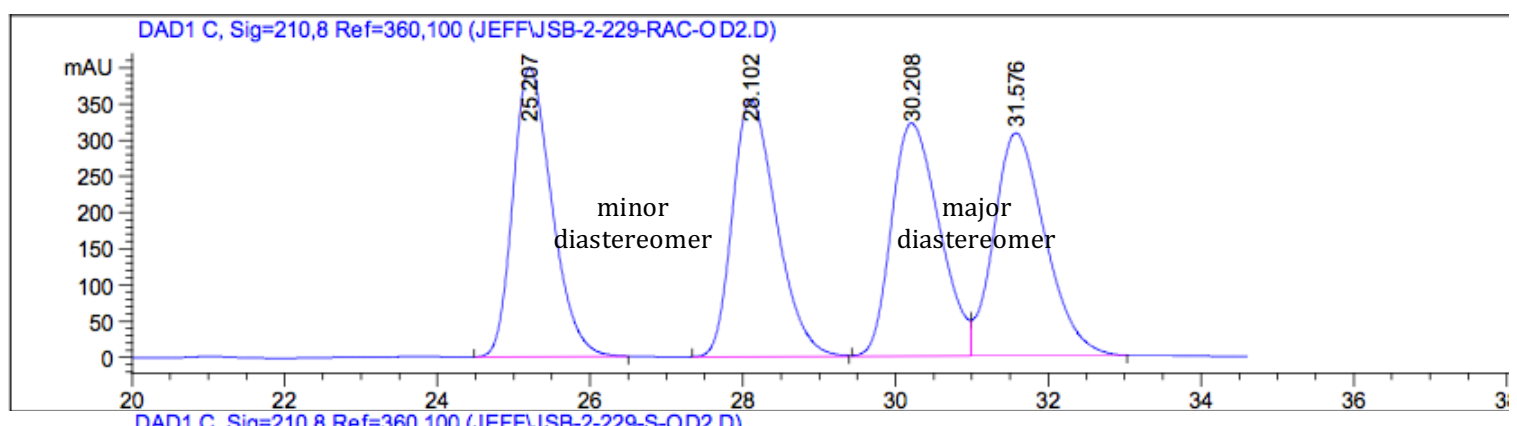

DAD1 C, Sig=210,8 Ref=360,100 (JEFFWSB-2-229-S-OD2.D)

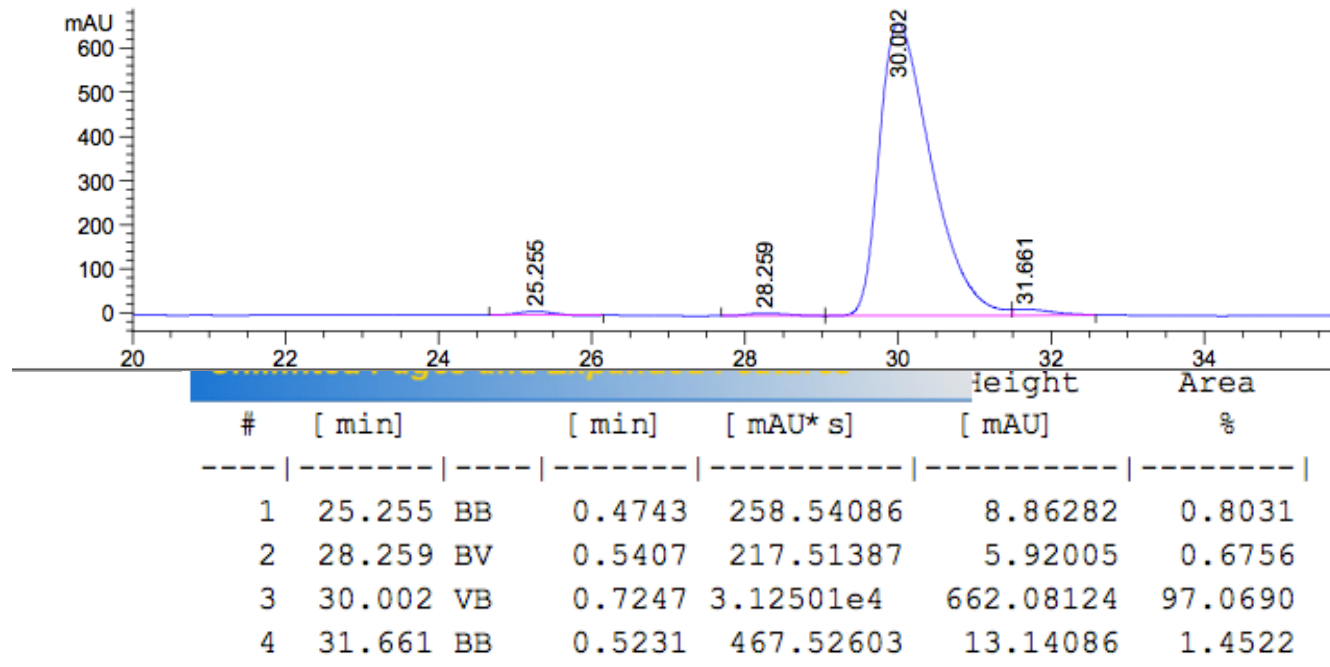

Totals :

$3.21936 \mathrm{e} 4 \quad 690.00497$ 
Scheme 1b, Reduction of Ketone 3a with $(R, R)$-Ph-BPE. Hexanes/iPrOH 99/1, 1.0 $\mathrm{mL} / \mathrm{min}, 210 \mathrm{~nm}, \mathrm{OD}-\mathrm{H}$.
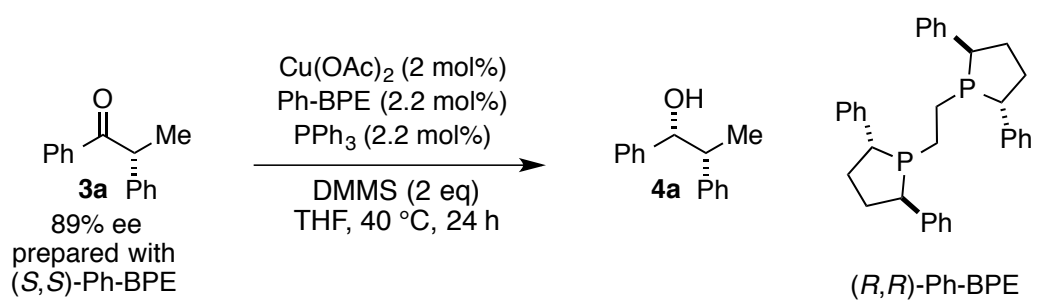

$(R, R)$-Ph-BPE

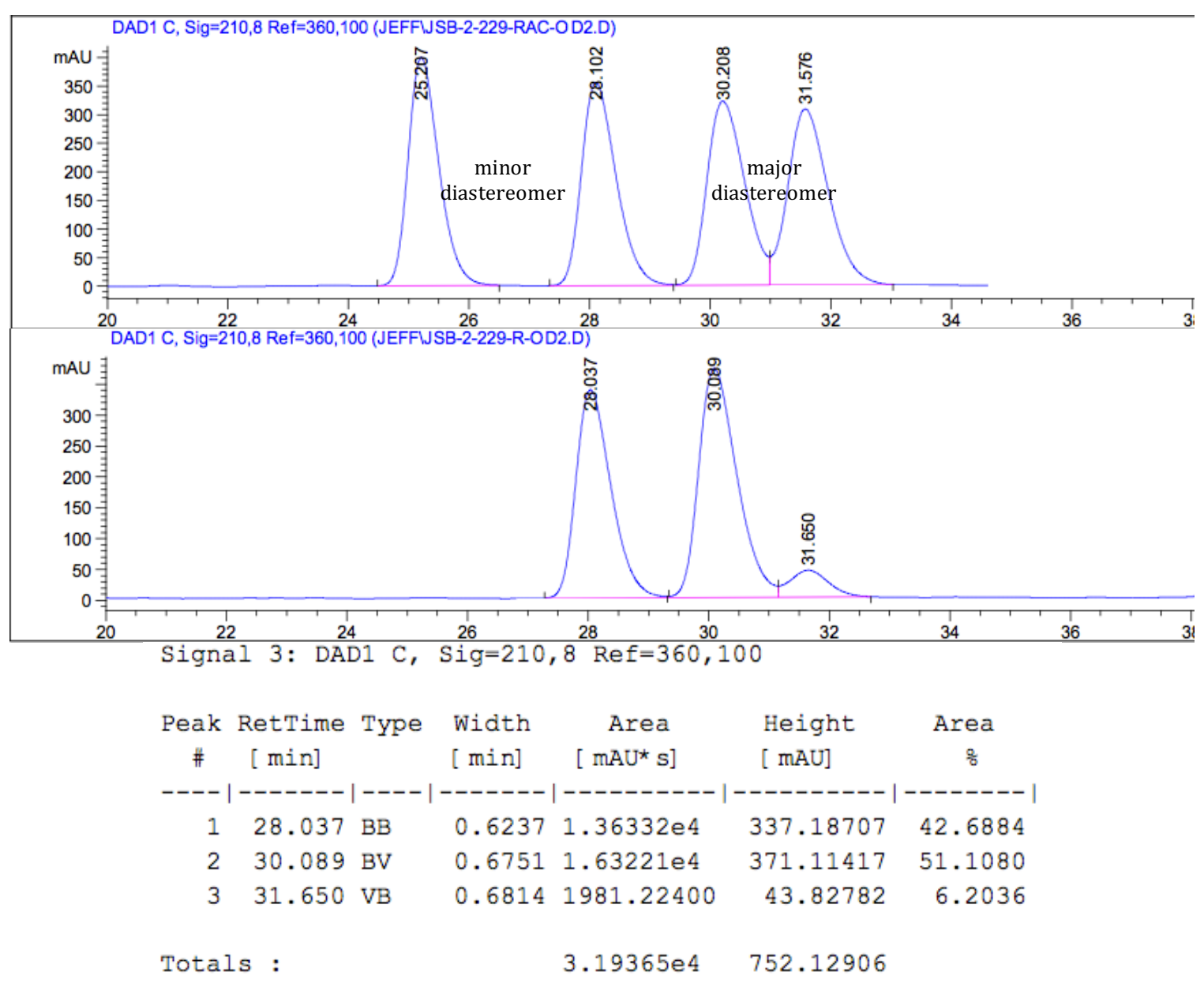

\section{NMR Data}

Note: For alcohol products, the minor diastereomer is visible around $1.3-1.4 \mathrm{ppm}\left(-\mathrm{CH}_{3}\right.$ group). This chemical shift was used to determine the diastereoselectivity on the crude reaction material (see General Procedure for details). 
J. Am. Chem. Soc. Supporting Information

$S 40$

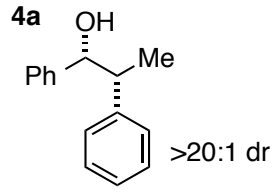

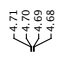

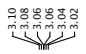

พ

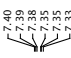

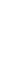

$4 a$

年

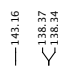

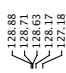

篦

ำ

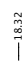
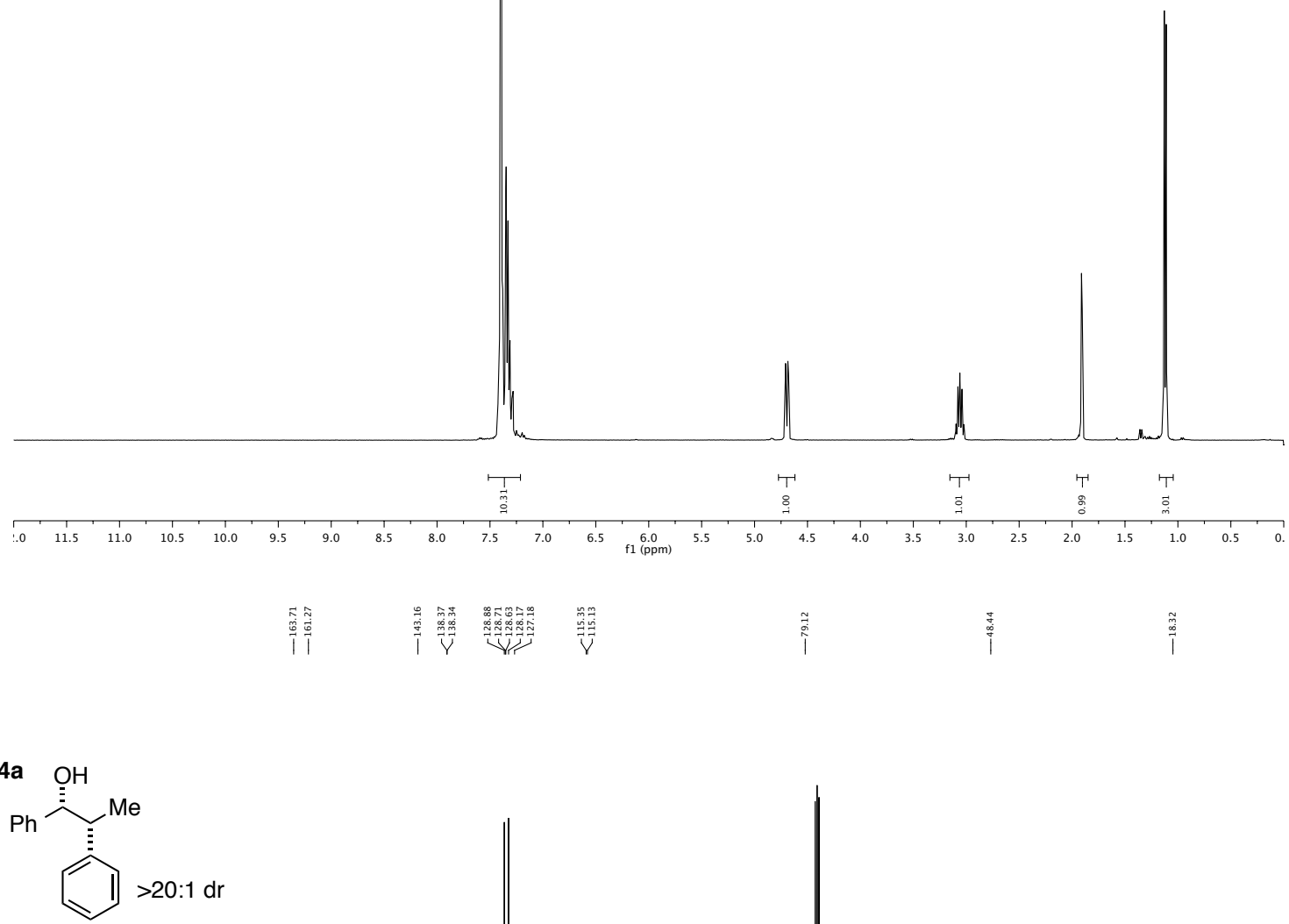

|

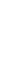
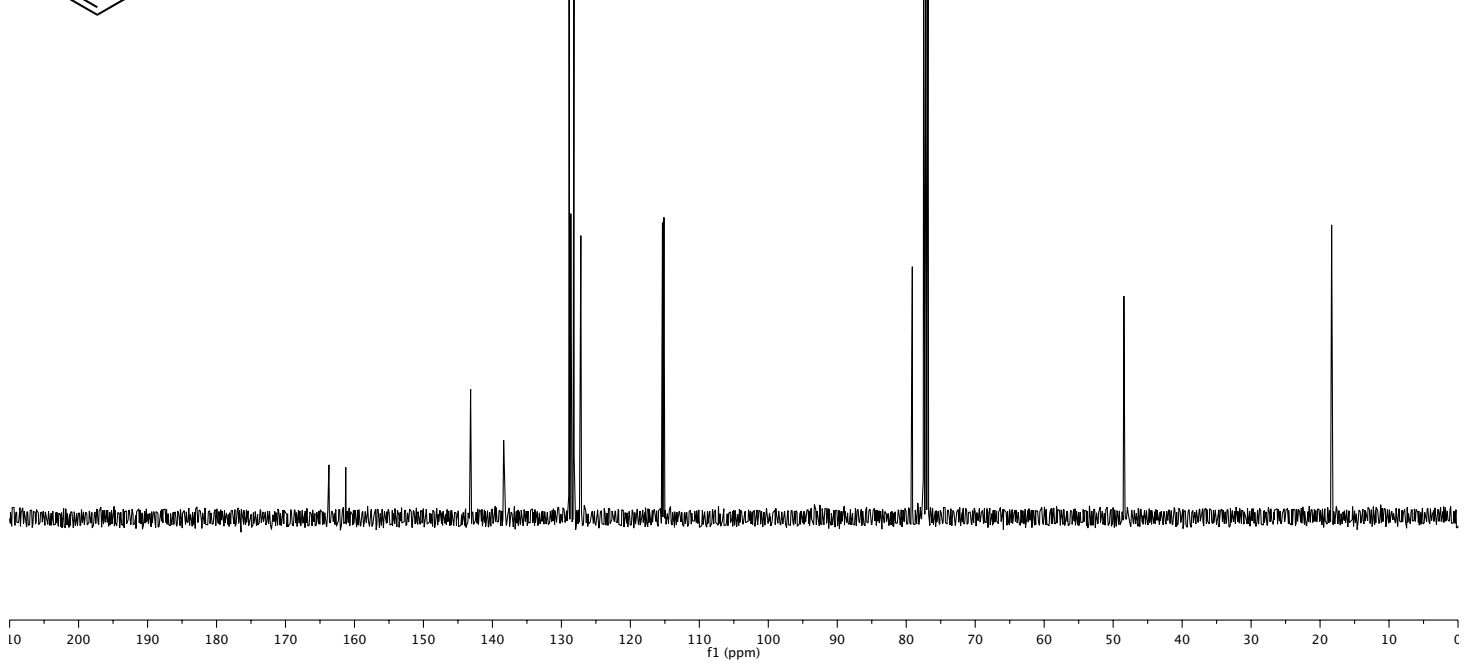


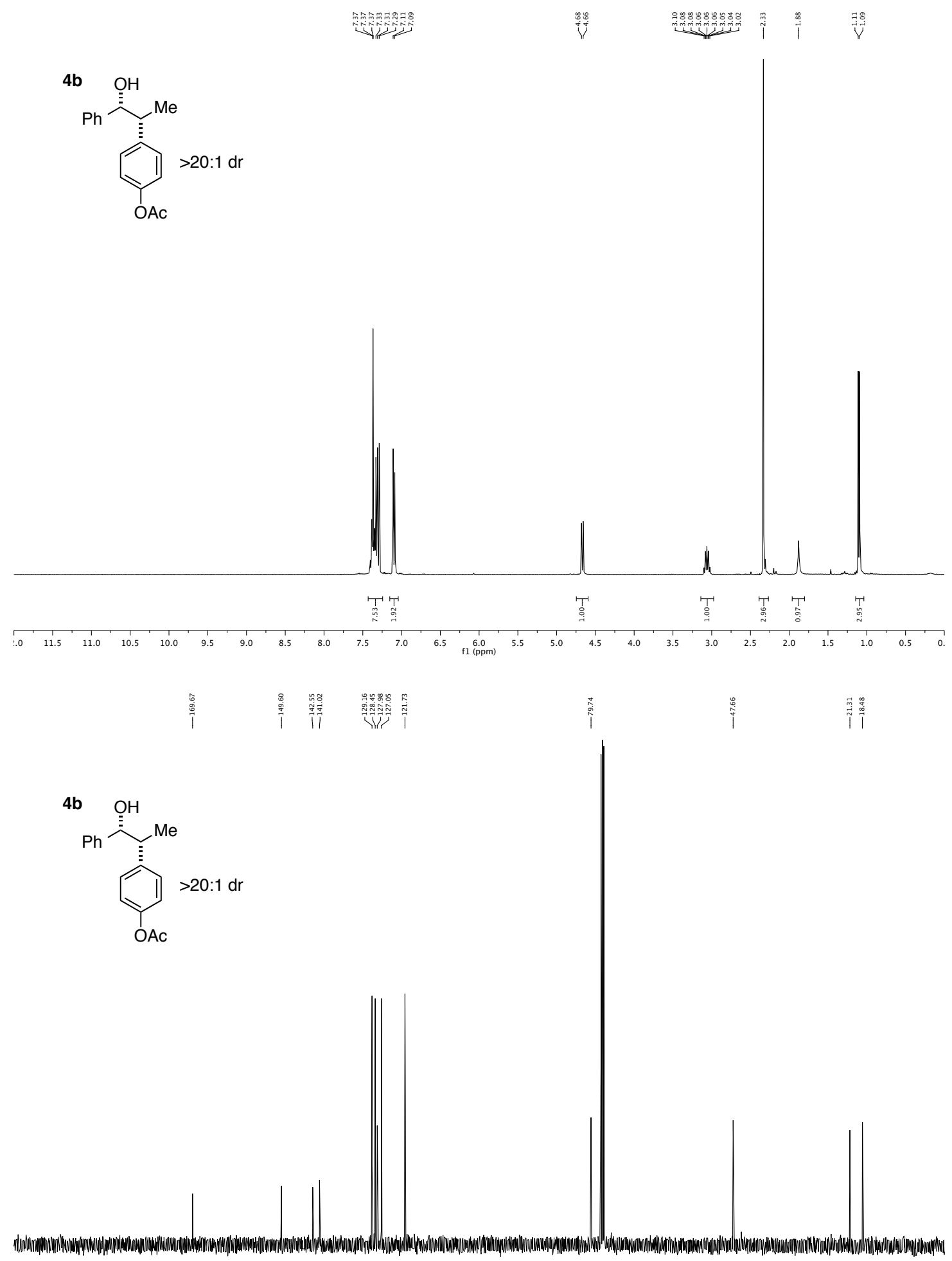

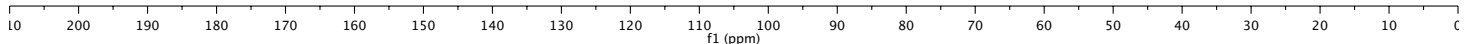


4c
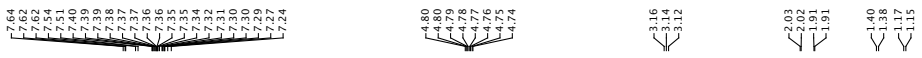

4c<smiles>C[C@H](c1ccccc1)[C@H](C)[C@H](C)c1ccc(C#N)cc1</smiles>

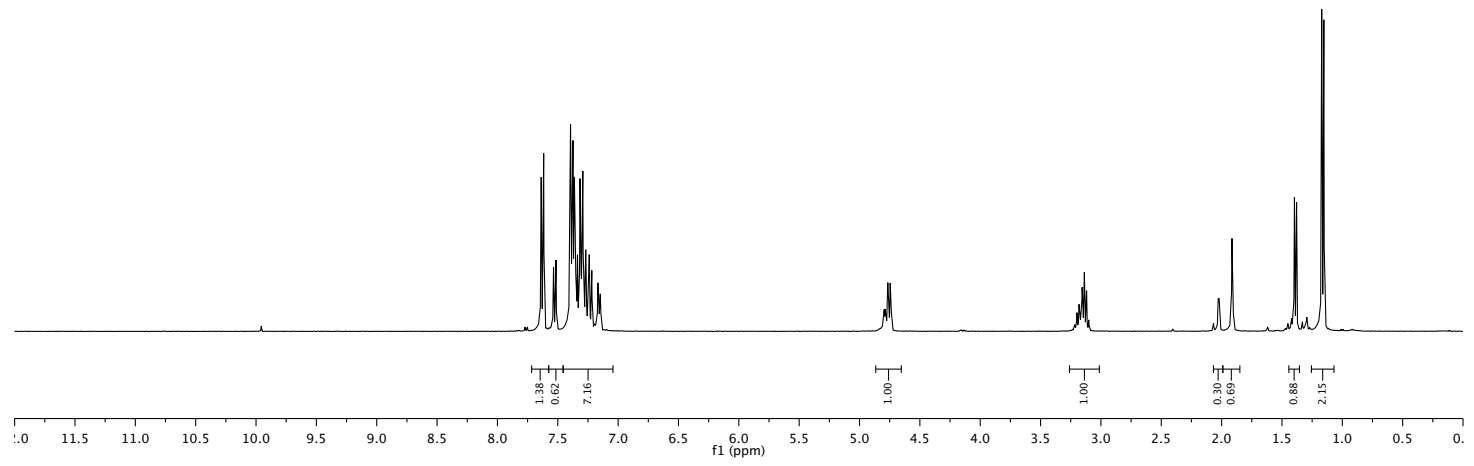

$4 c$
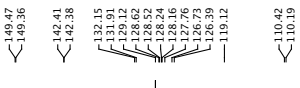

$8 \%$
80
11
11

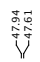

$\stackrel{\substack{0 \\ \infty}}{\substack{0 \\ 0}}$<smiles>C[C@H](c1ccc(C#N)cc1)[C@@H](O)c1ccccc1</smiles>

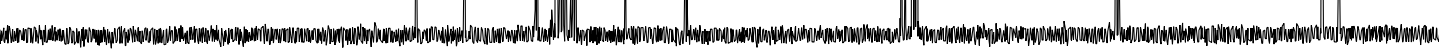

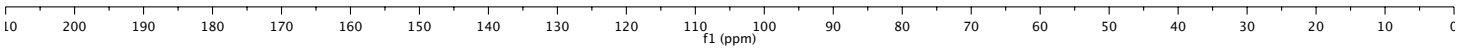




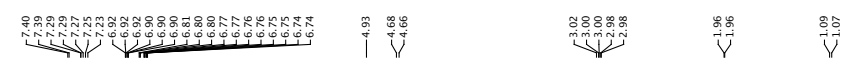<smiles>C[C@H](c1cccc(O)c1)C(O)c1ccccc1</smiles>

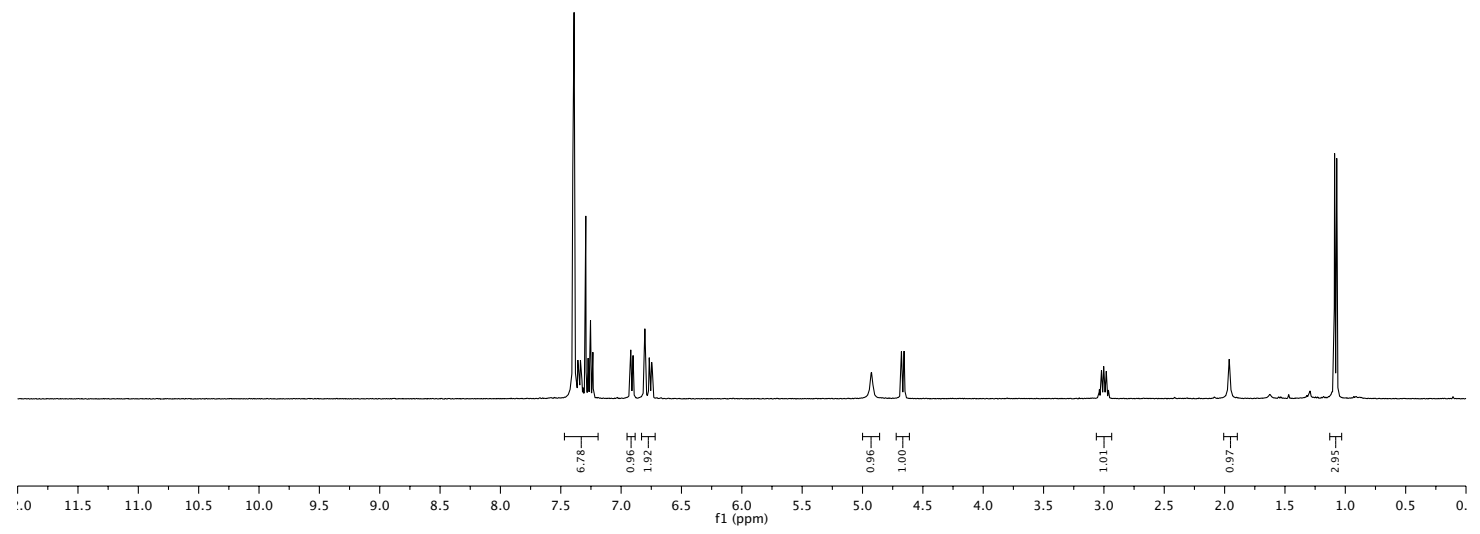

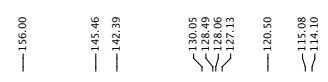
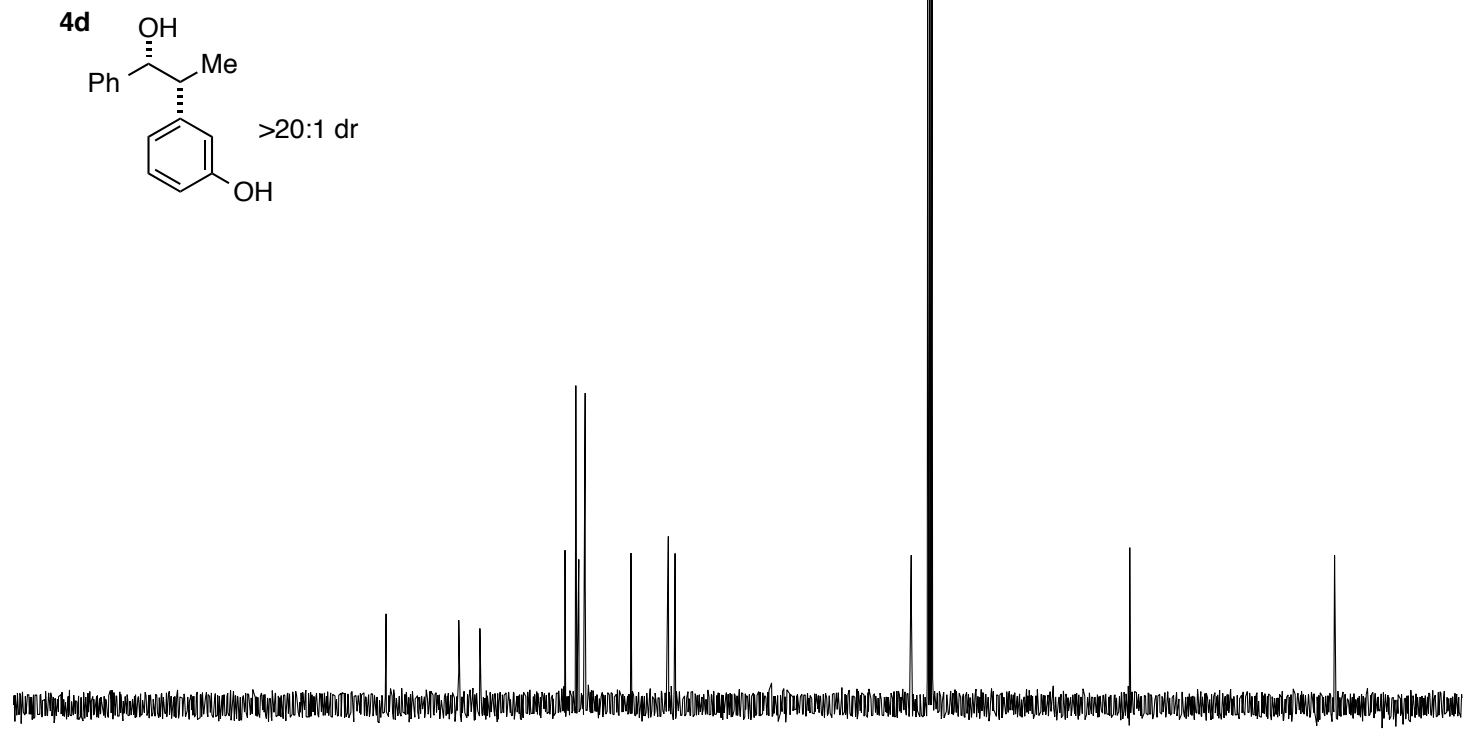

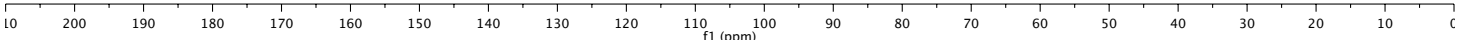




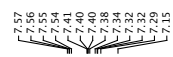

4e $\mathrm{OH}$<smiles>CC(Cc1ccccc1)c1ccc(/C=C/c2ccccc2)cc1</smiles>

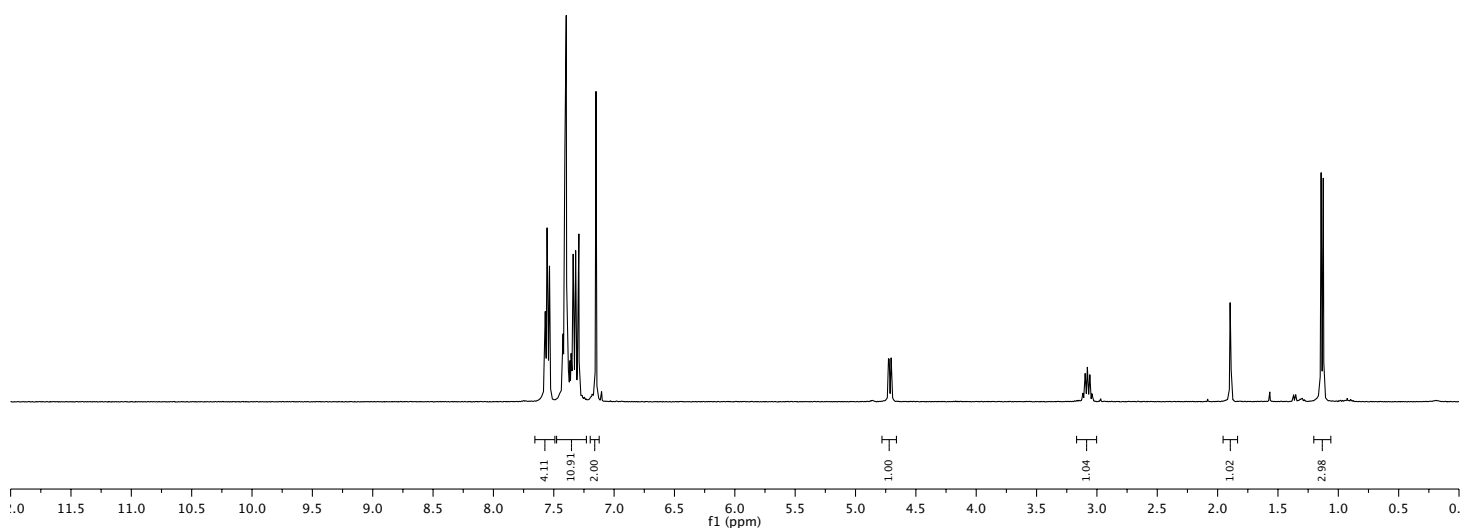

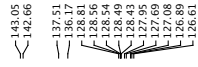

$4 \mathrm{e} \quad \mathrm{OH}$<smiles>O=[N+]([Al])c1ccc(C=Cc2ccccc2)cc1</smiles>

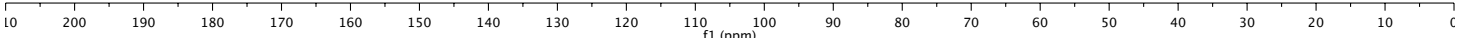



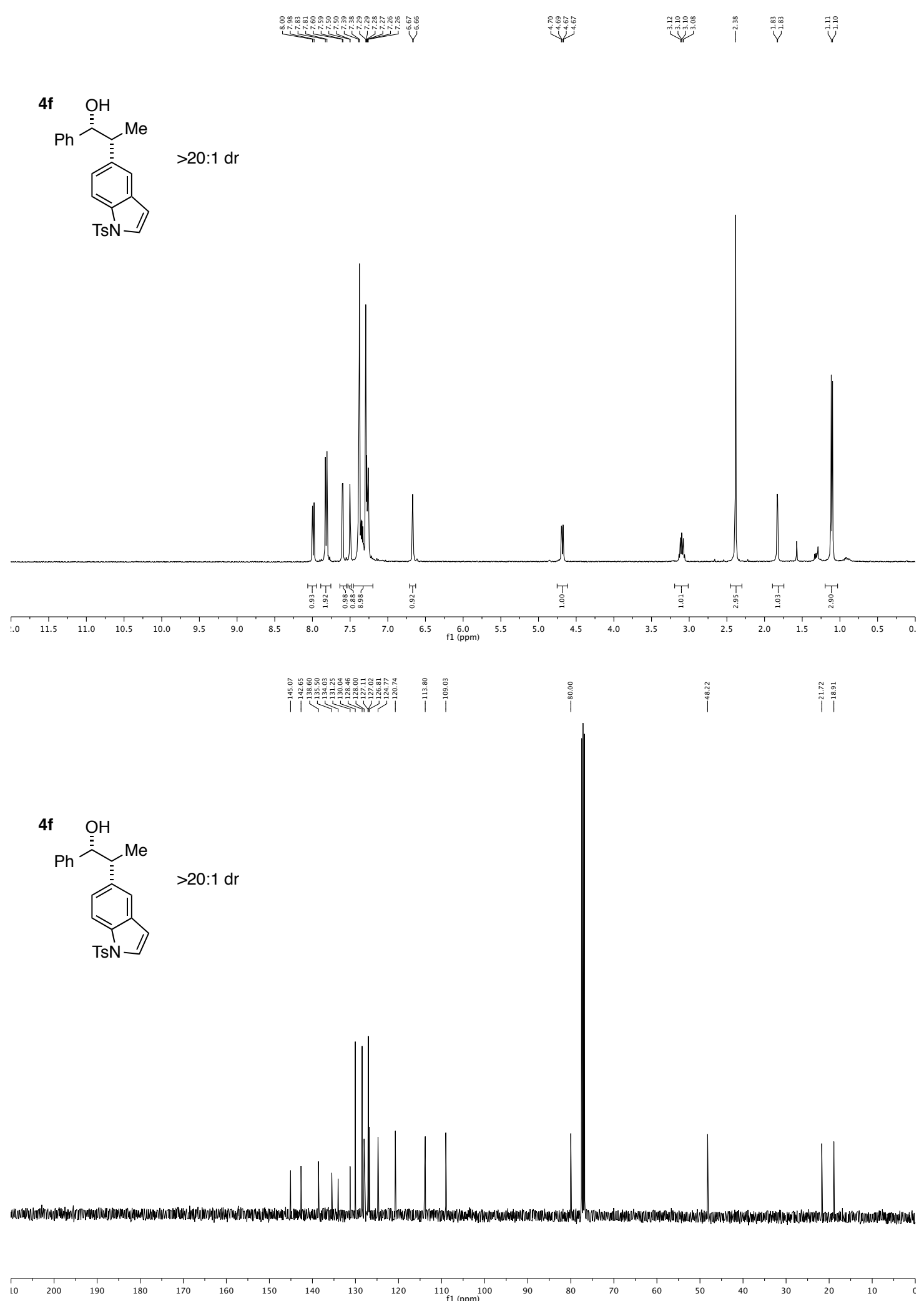

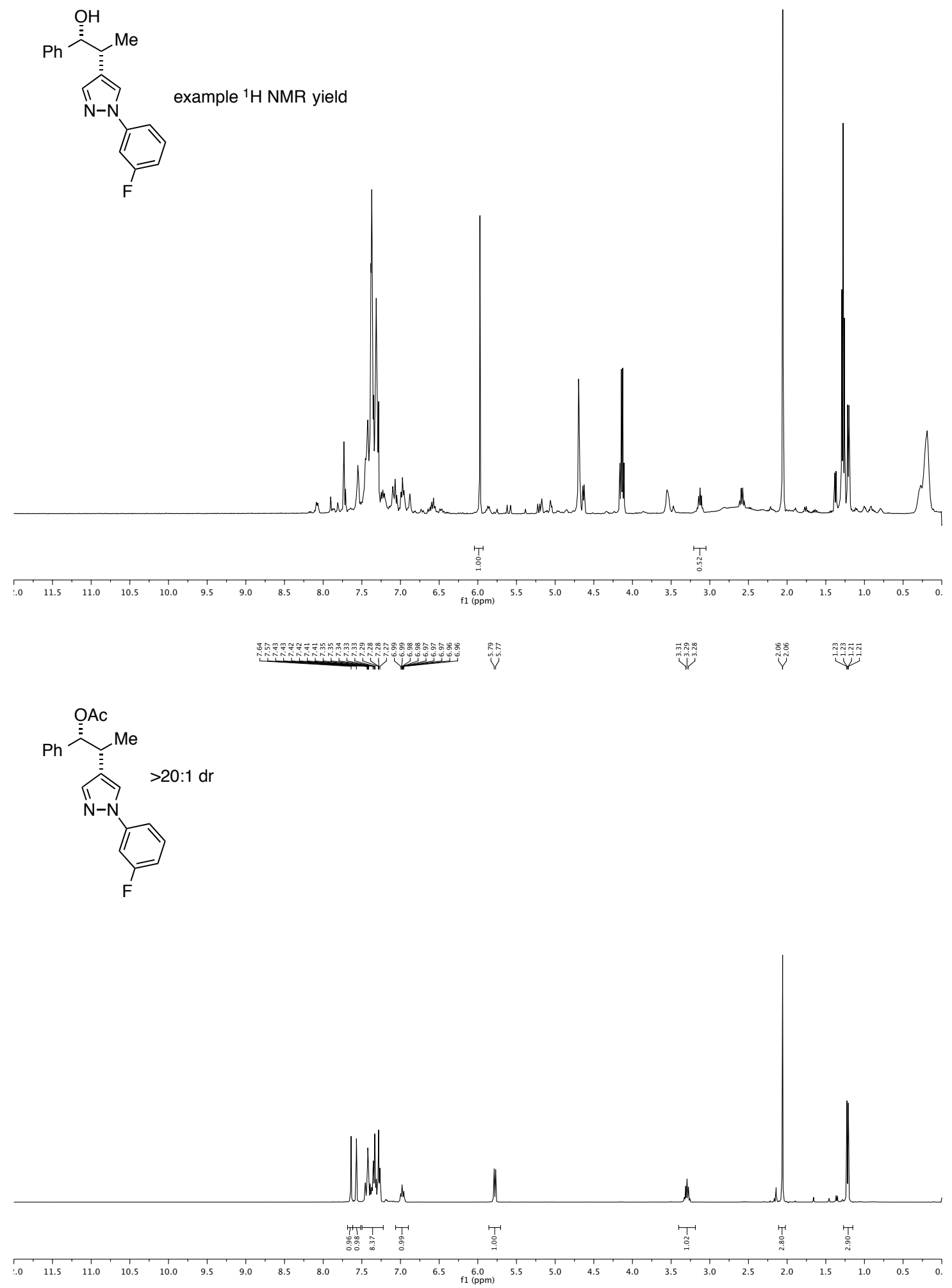


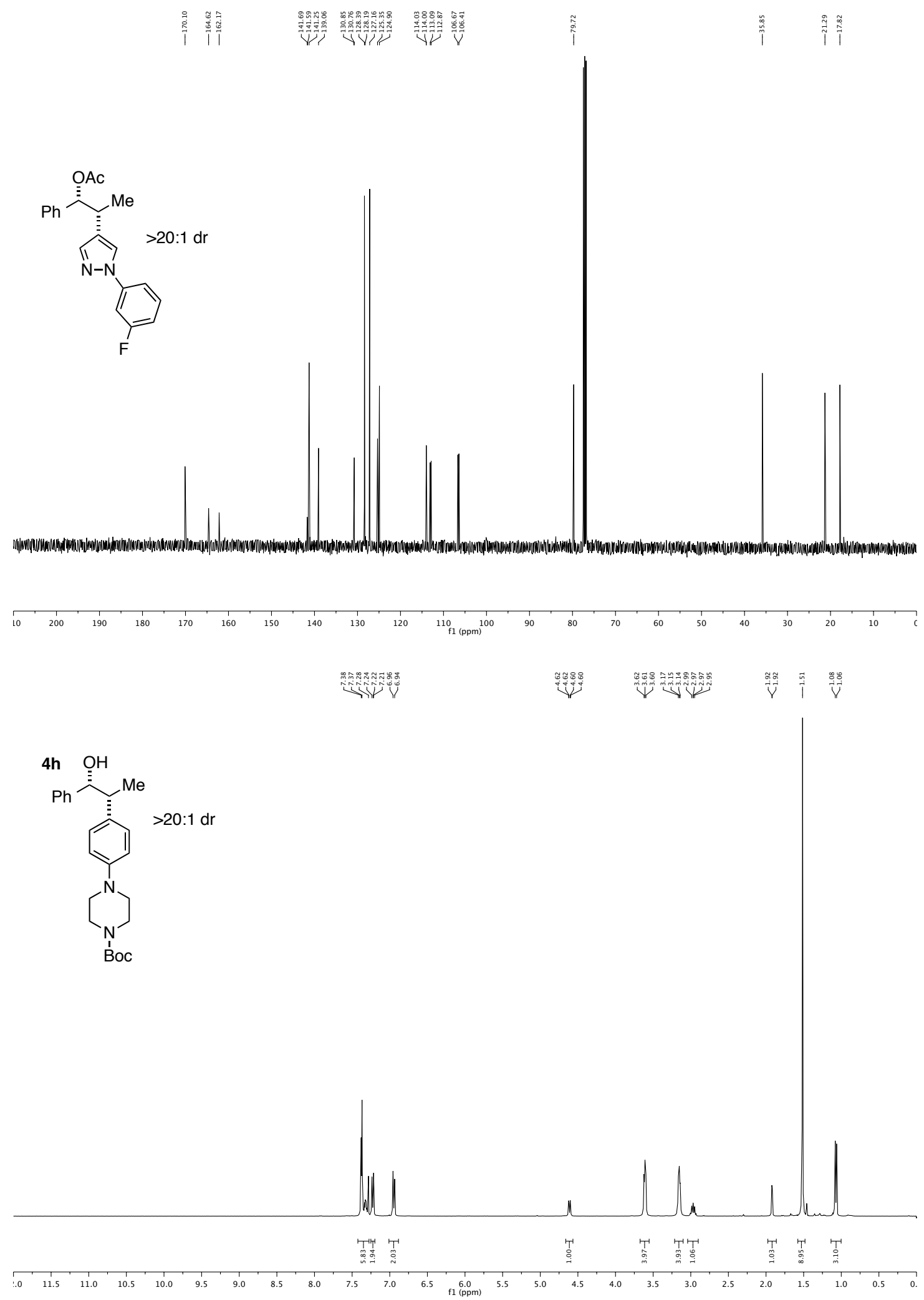


J. Am. Chem. Soc. Supporting Information

$S 48$

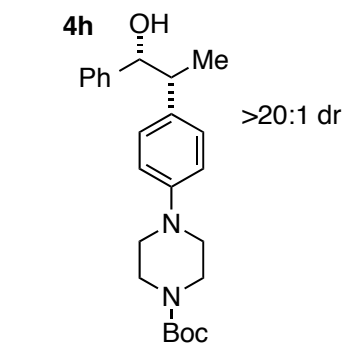

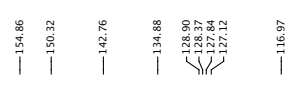

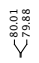

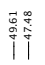
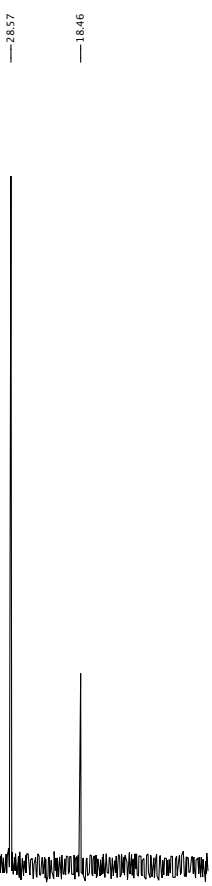

10

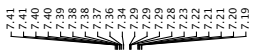

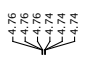

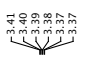

Vับ

$4 i$
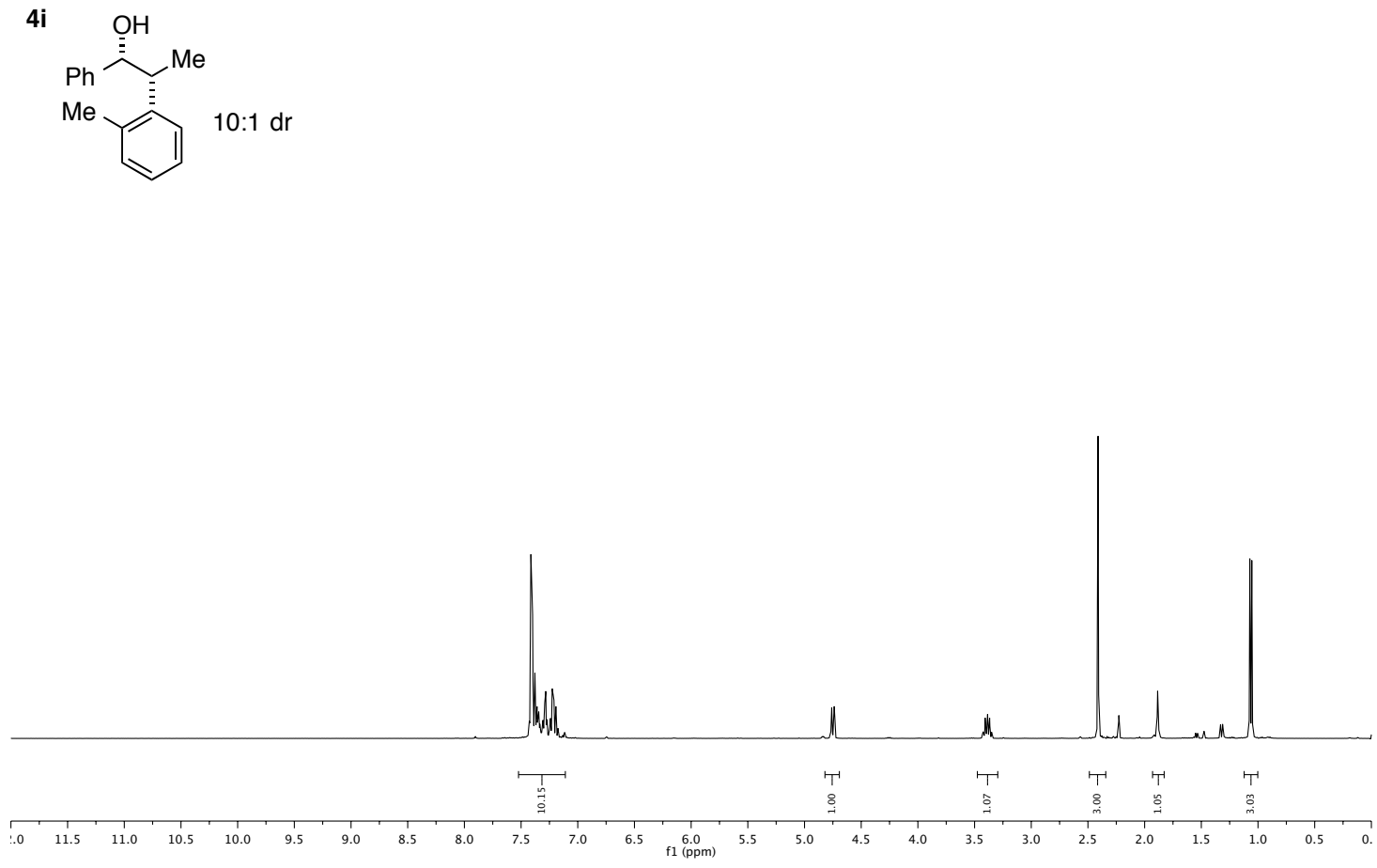

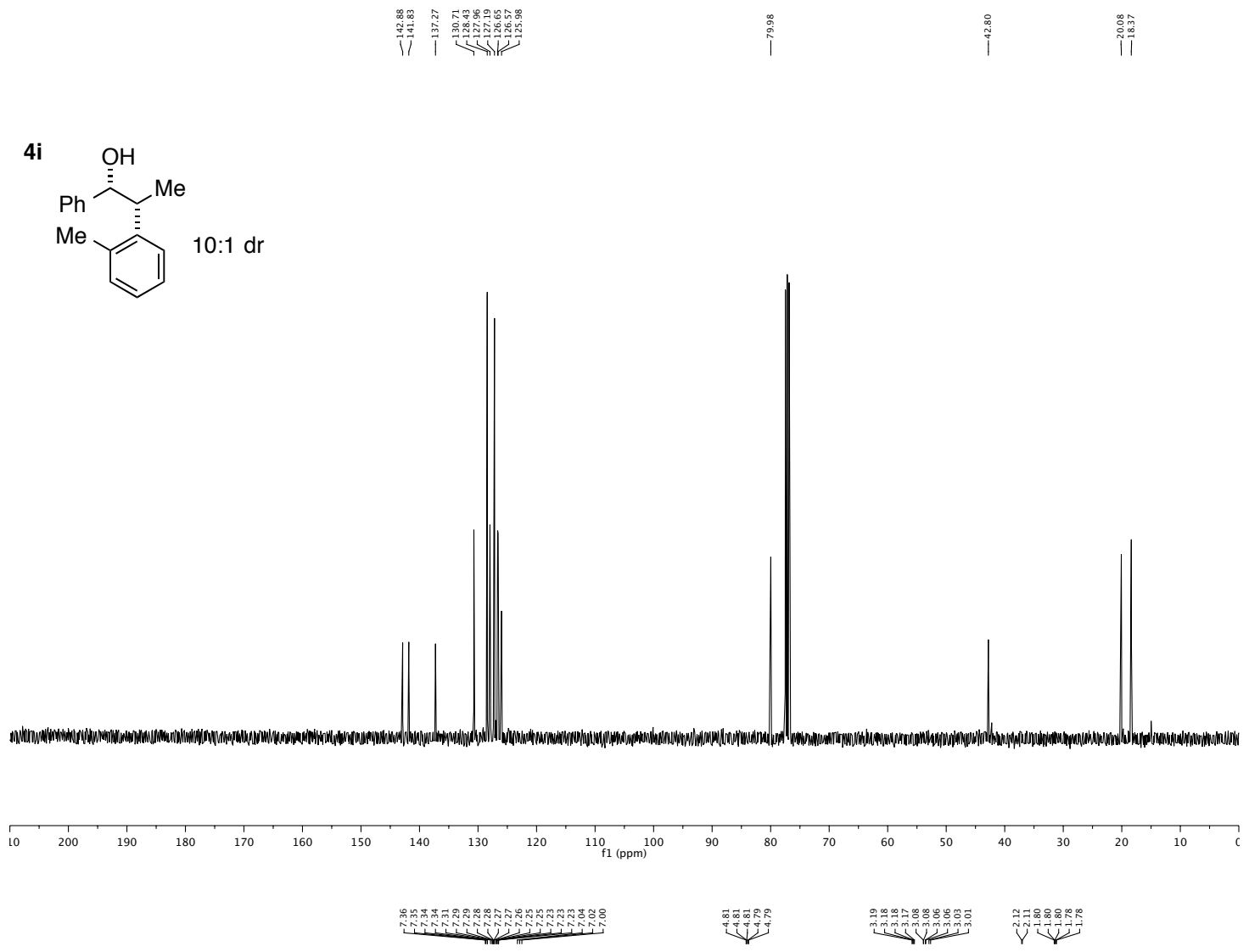

$\overbrace{>20: 1 \mathrm{dr}}^{4 j}$

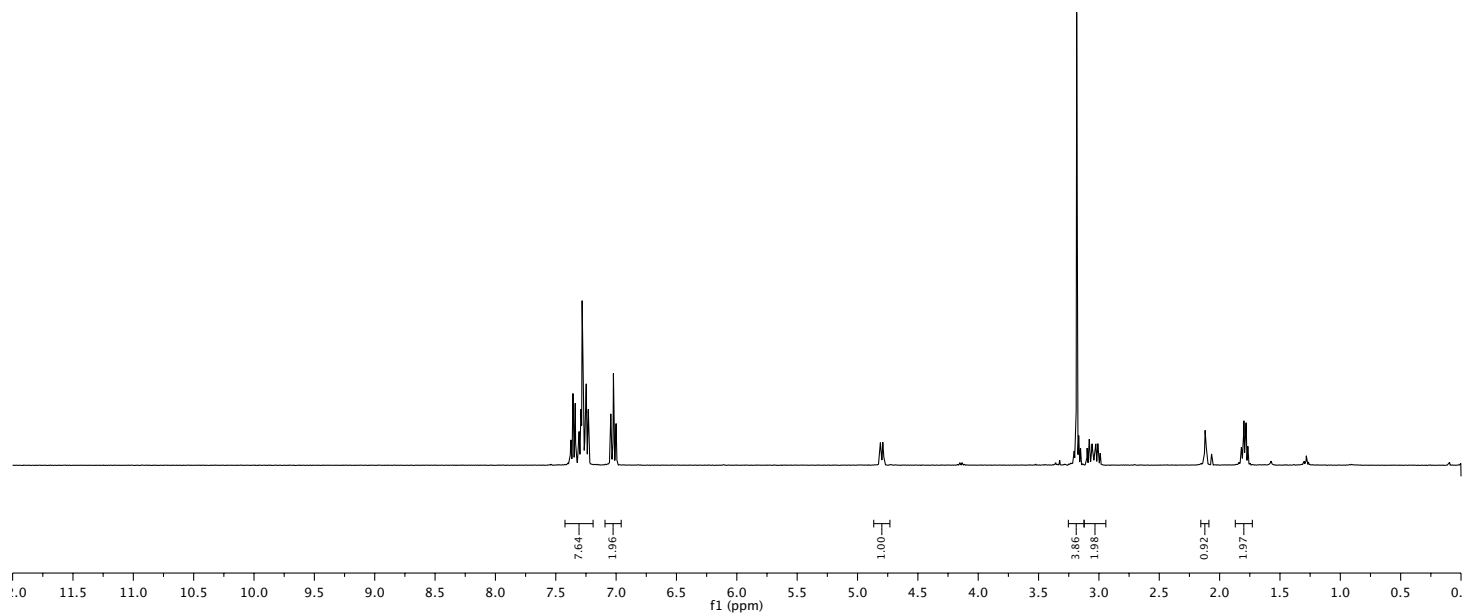




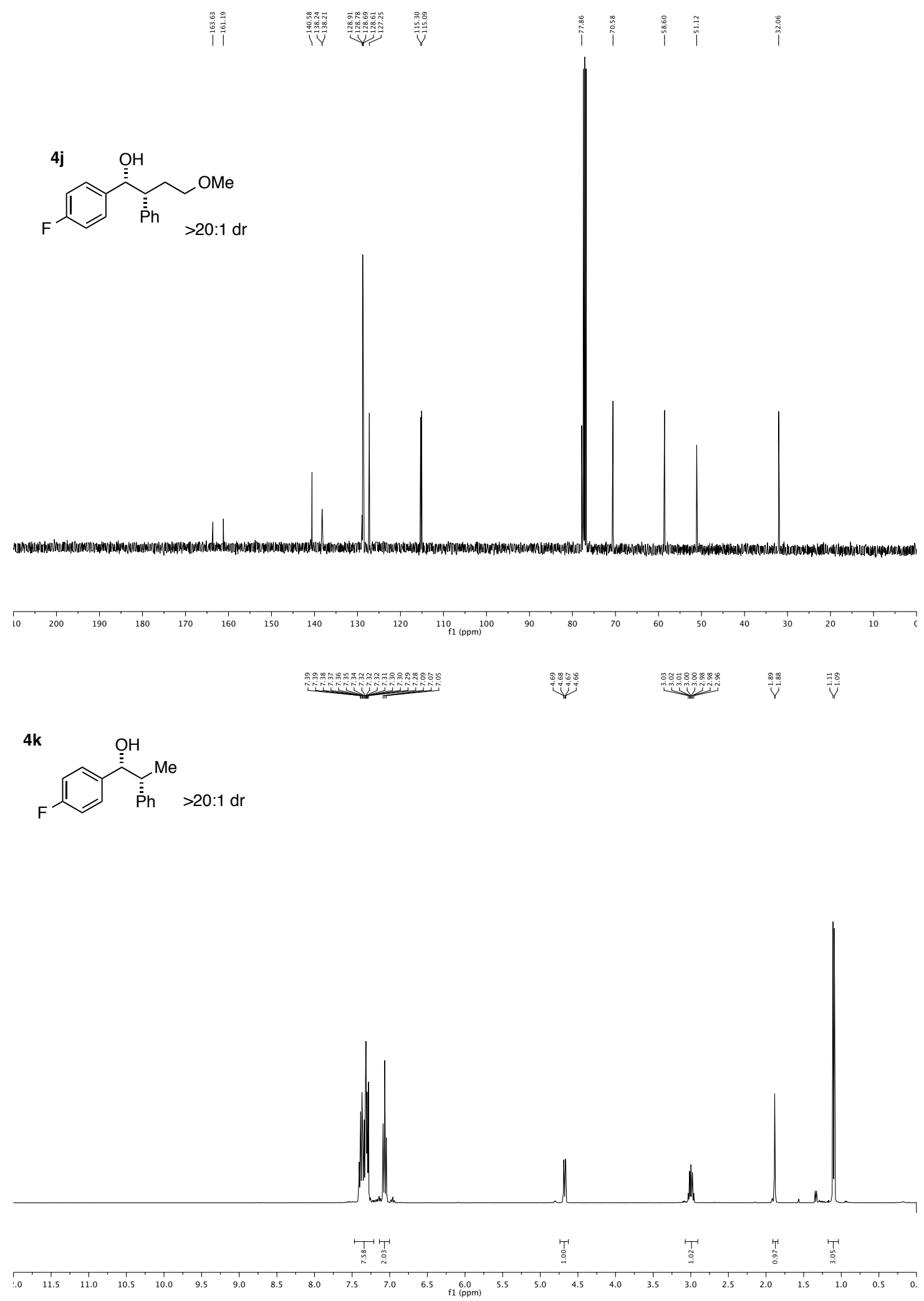



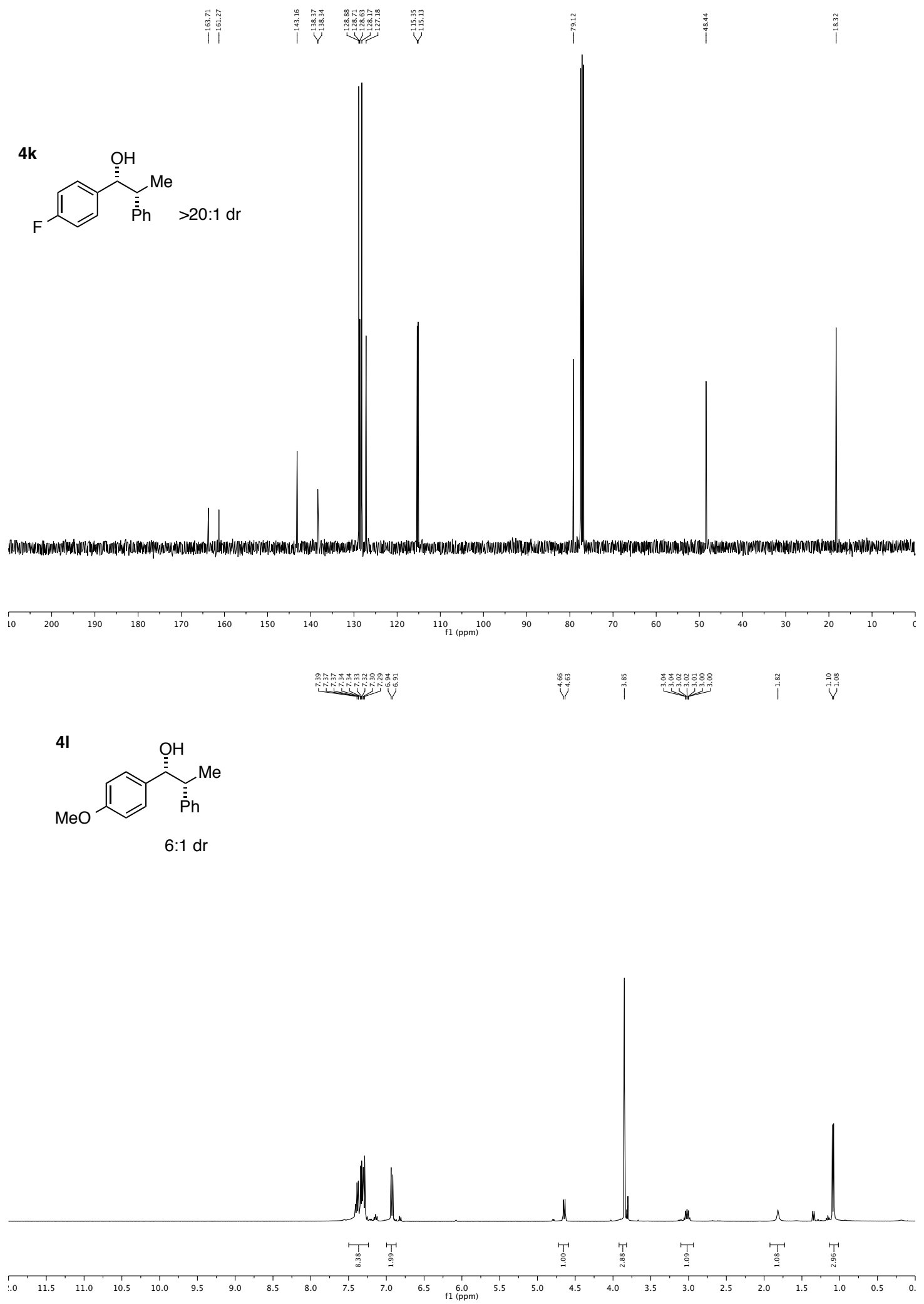
<smiles>COc1ccc(C(O)C(C)c2ccccc2)cc1</smiles>

$6: 1 \mathrm{dr}$

$4 \mathrm{~m}$<smiles>C[C@H](c1ccccc1Cl)[C@@H](O)c1ccccc1</smiles>

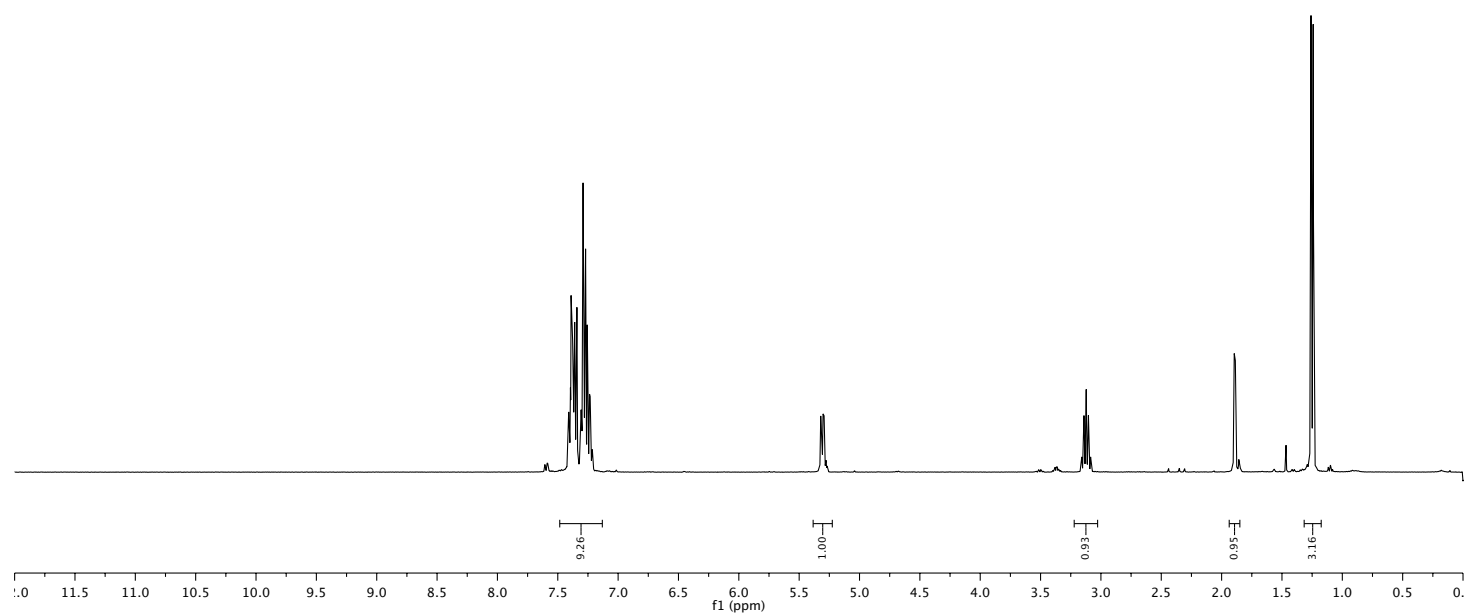




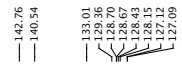

$4 m$
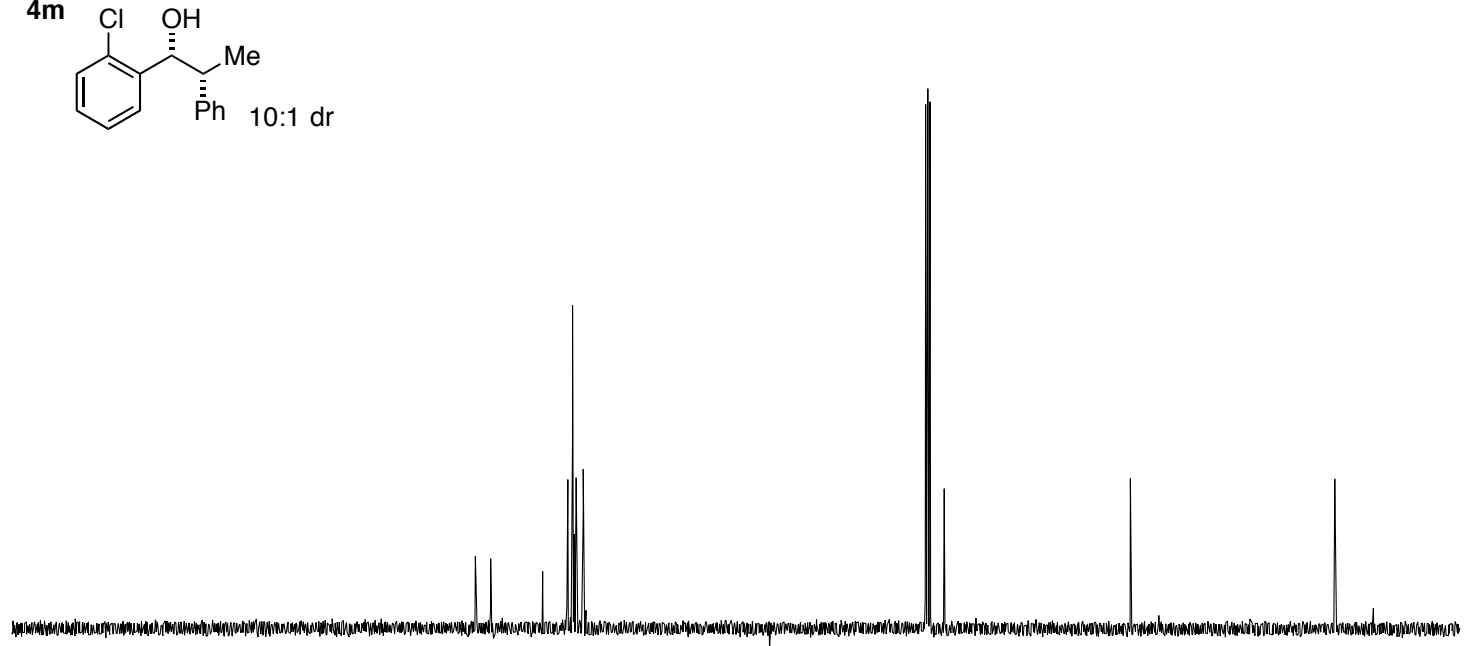

10
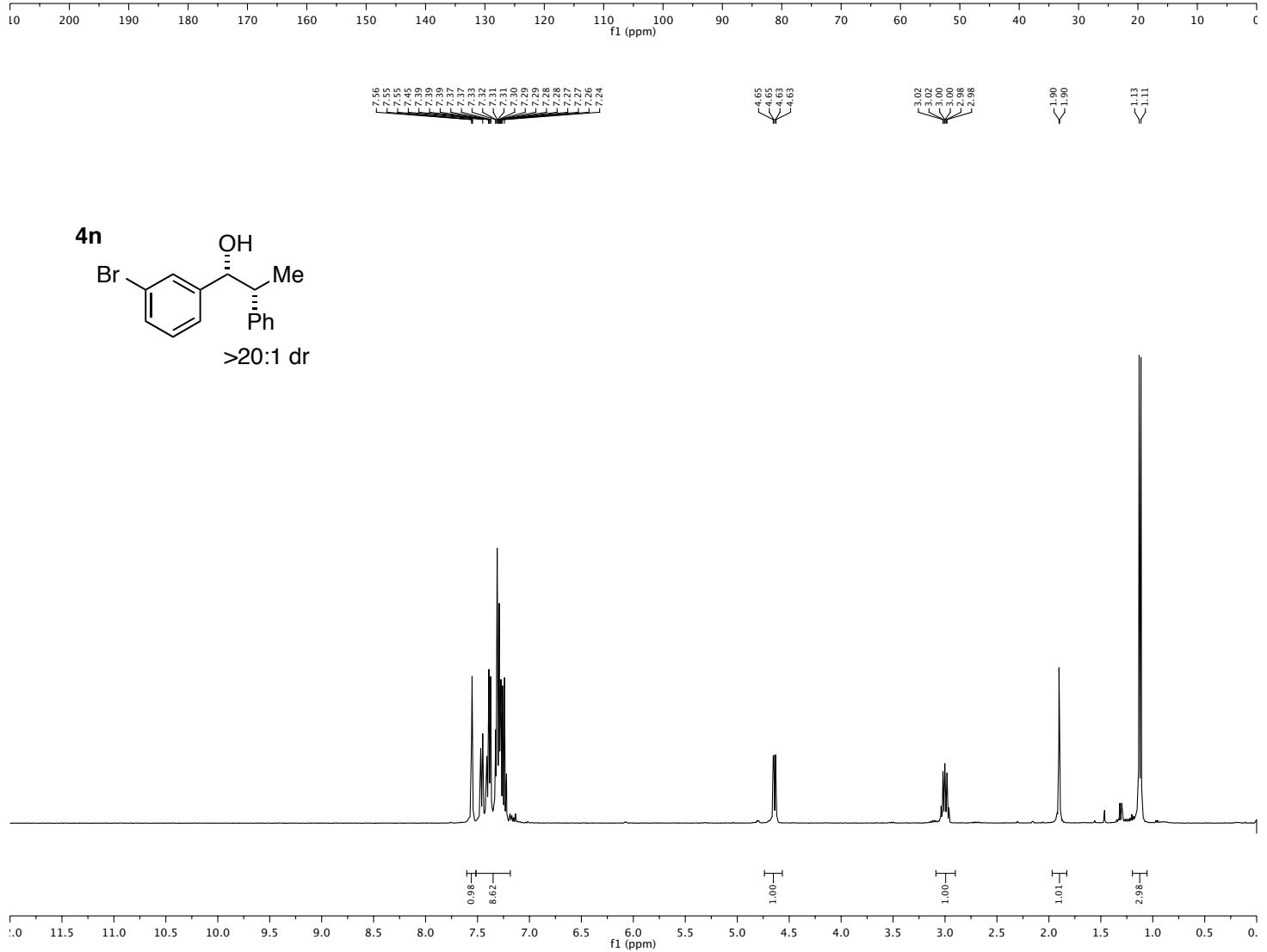


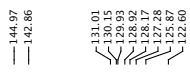

$4 n$
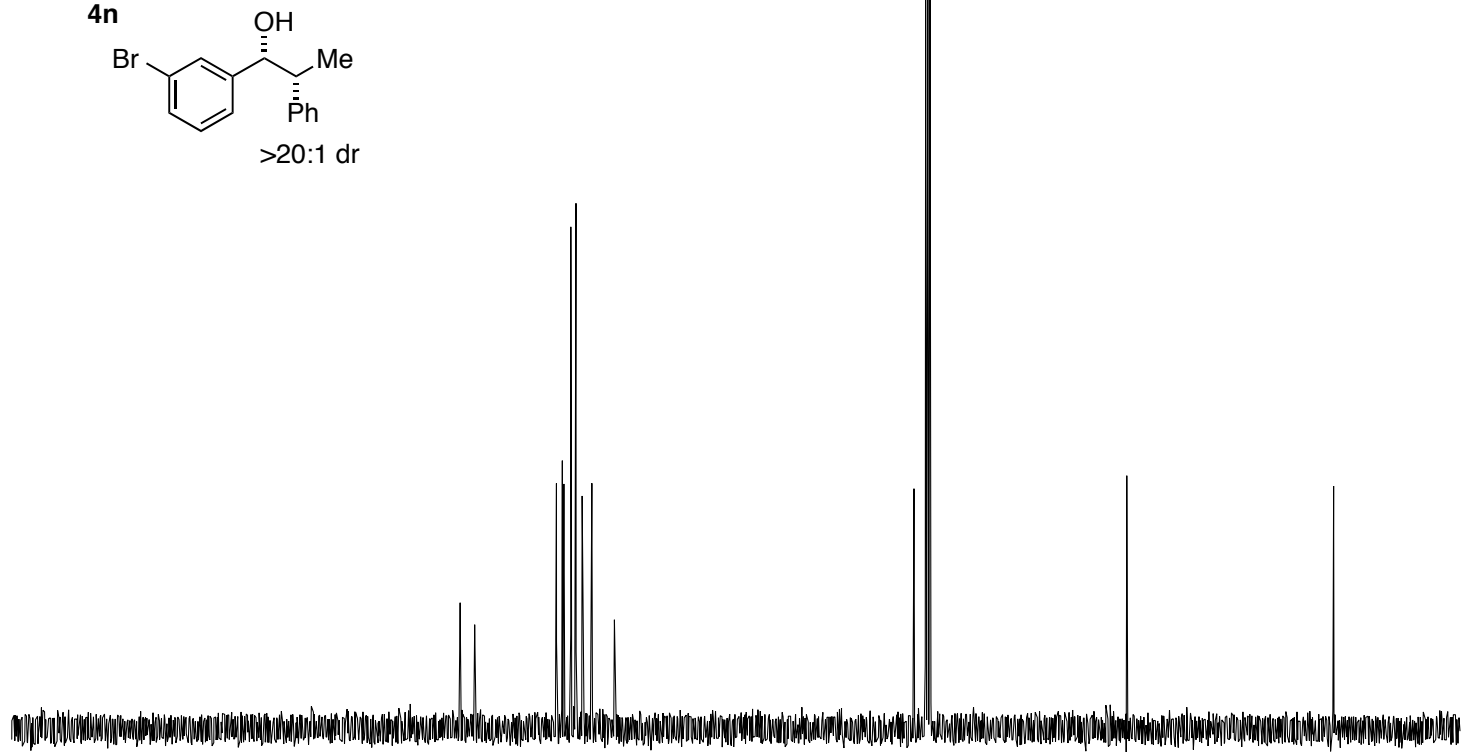

10
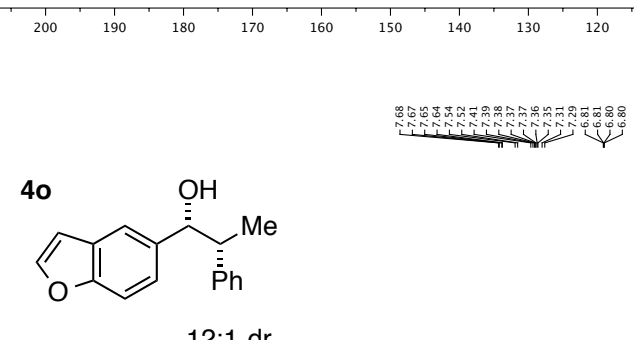

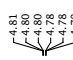

ע

40

$12: 1 \mathrm{dr}$

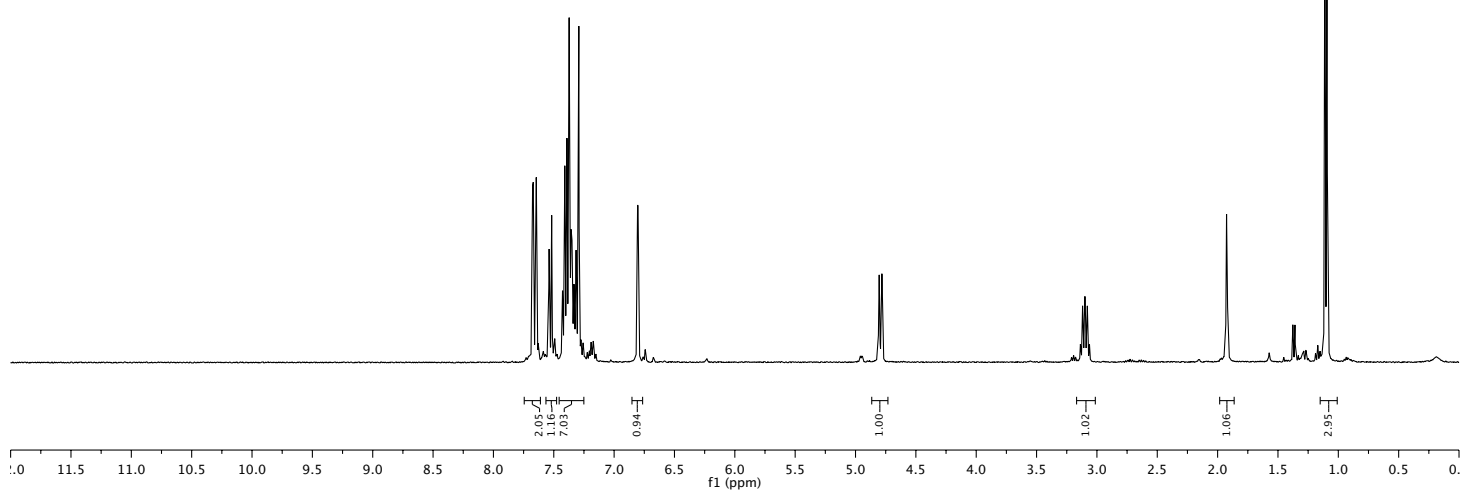




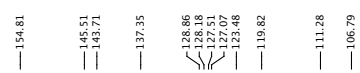

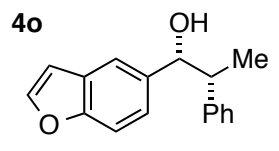

$12: 1 \mathrm{dr}$

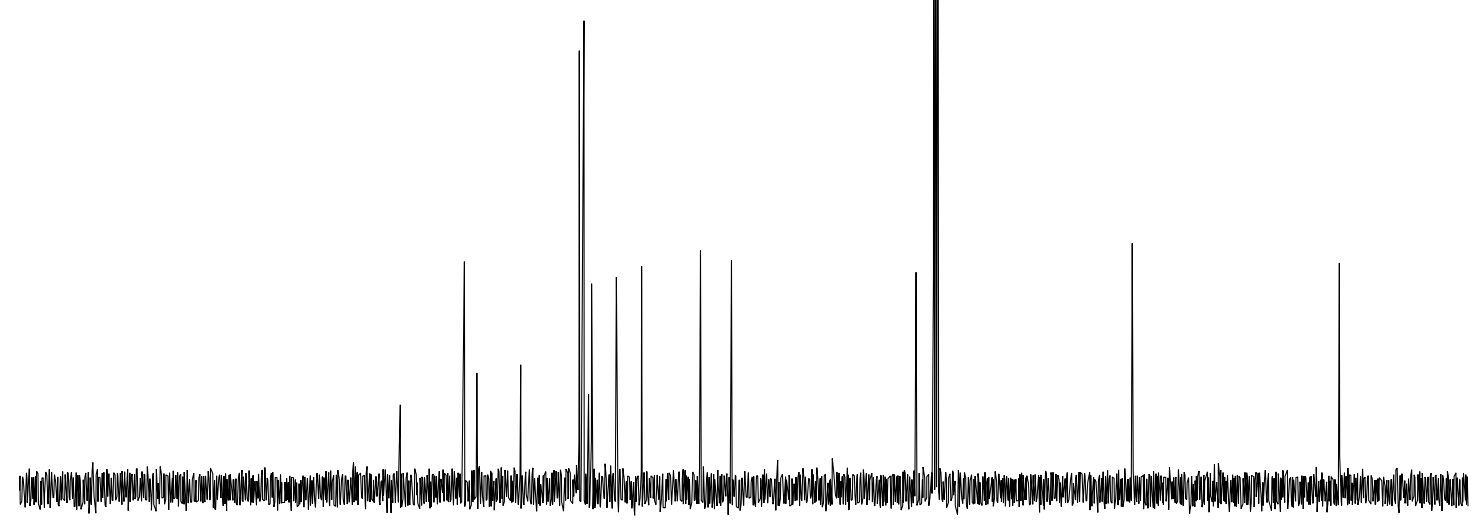

\begin{tabular}{rrrrr}
\hline 10 & 200 & 190 & 180 & 170
\end{tabular}

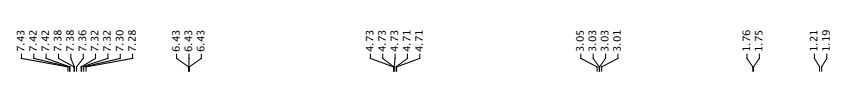

$4 p$
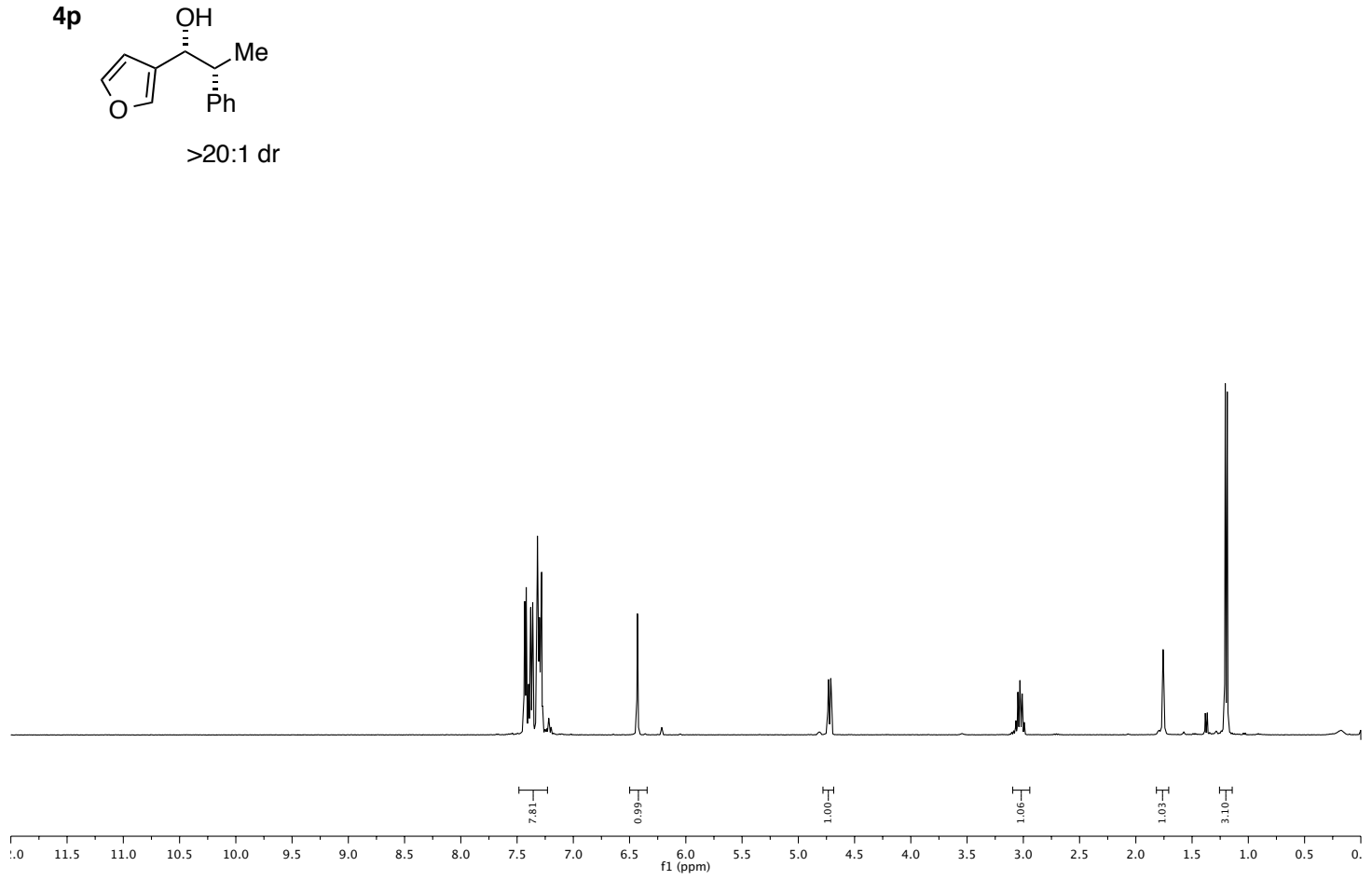


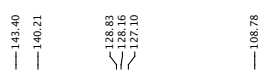

$4 p$

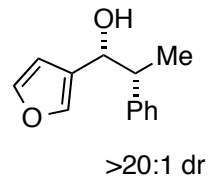

$>20: 1 \mathrm{dr}$

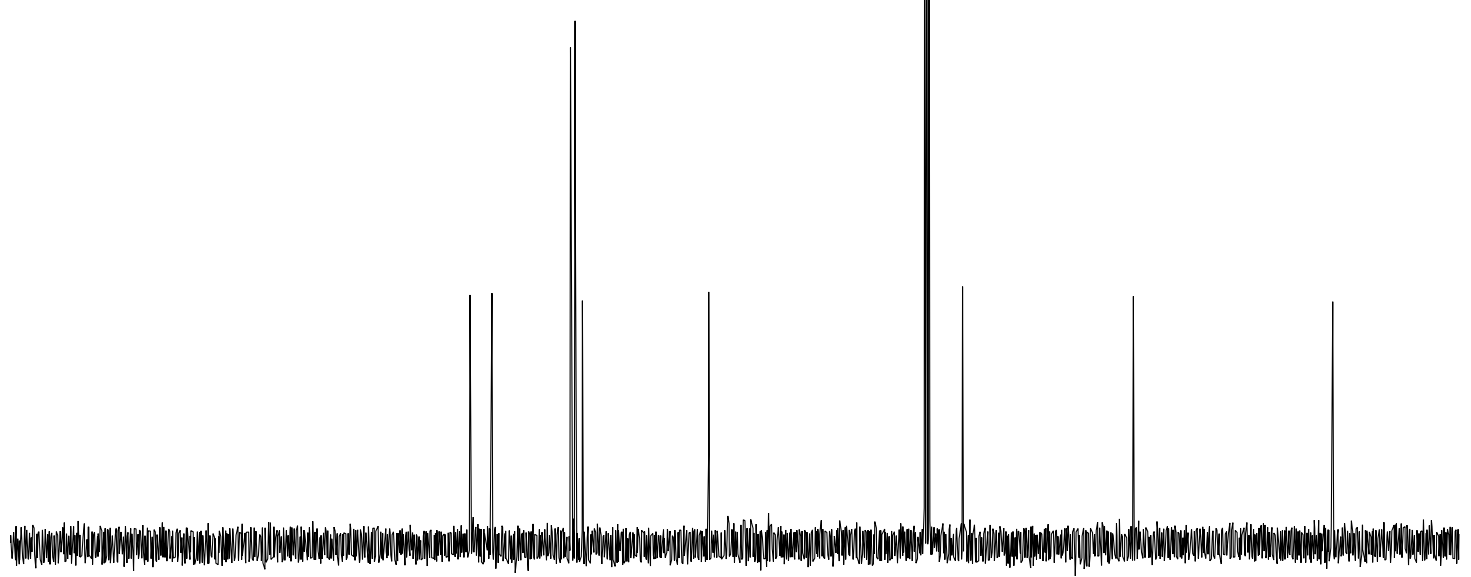

10
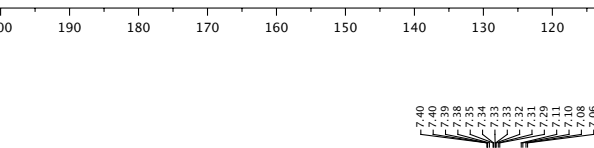

$\underbrace{\substack{\infty \\ j}}_{\substack{n=\infty \\ \infty}}$

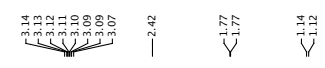

$4 q$
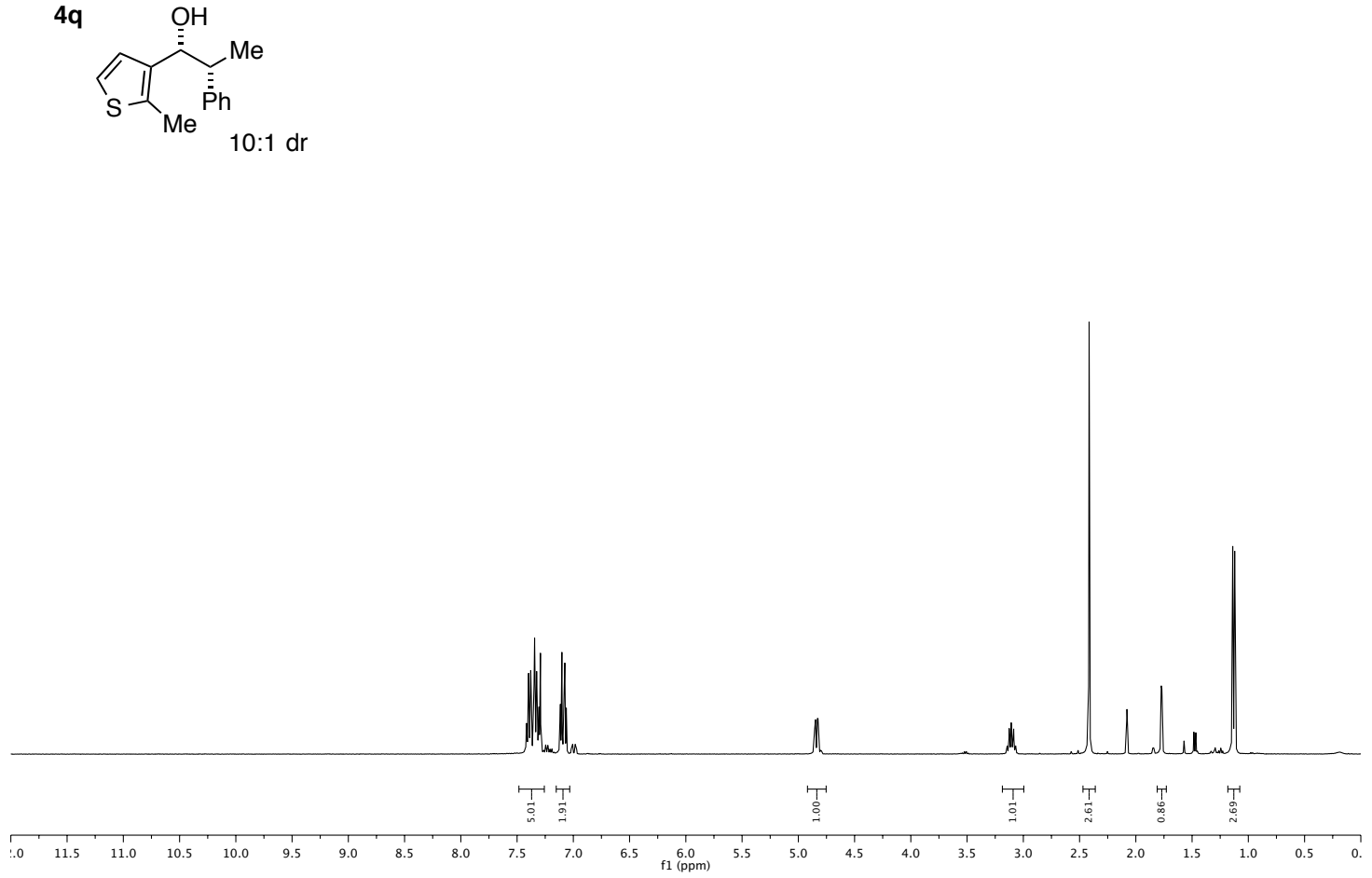


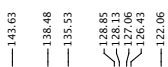

$4 q$
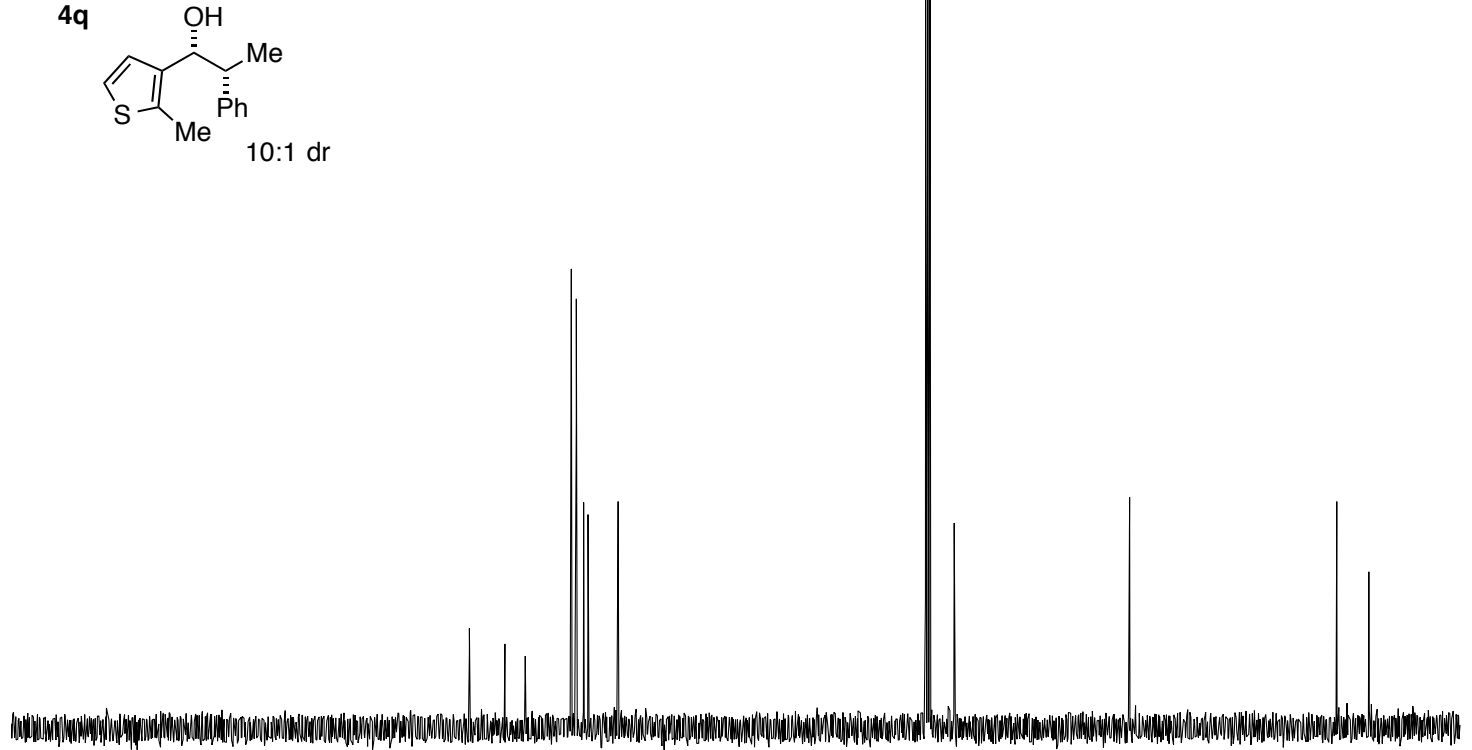

10
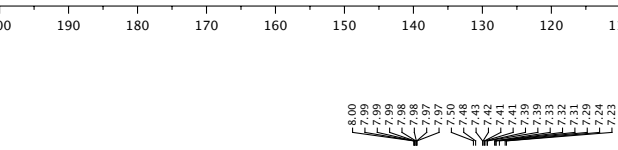

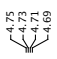

$\stackrel{2}{y}$

3a

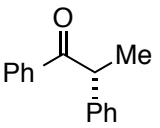
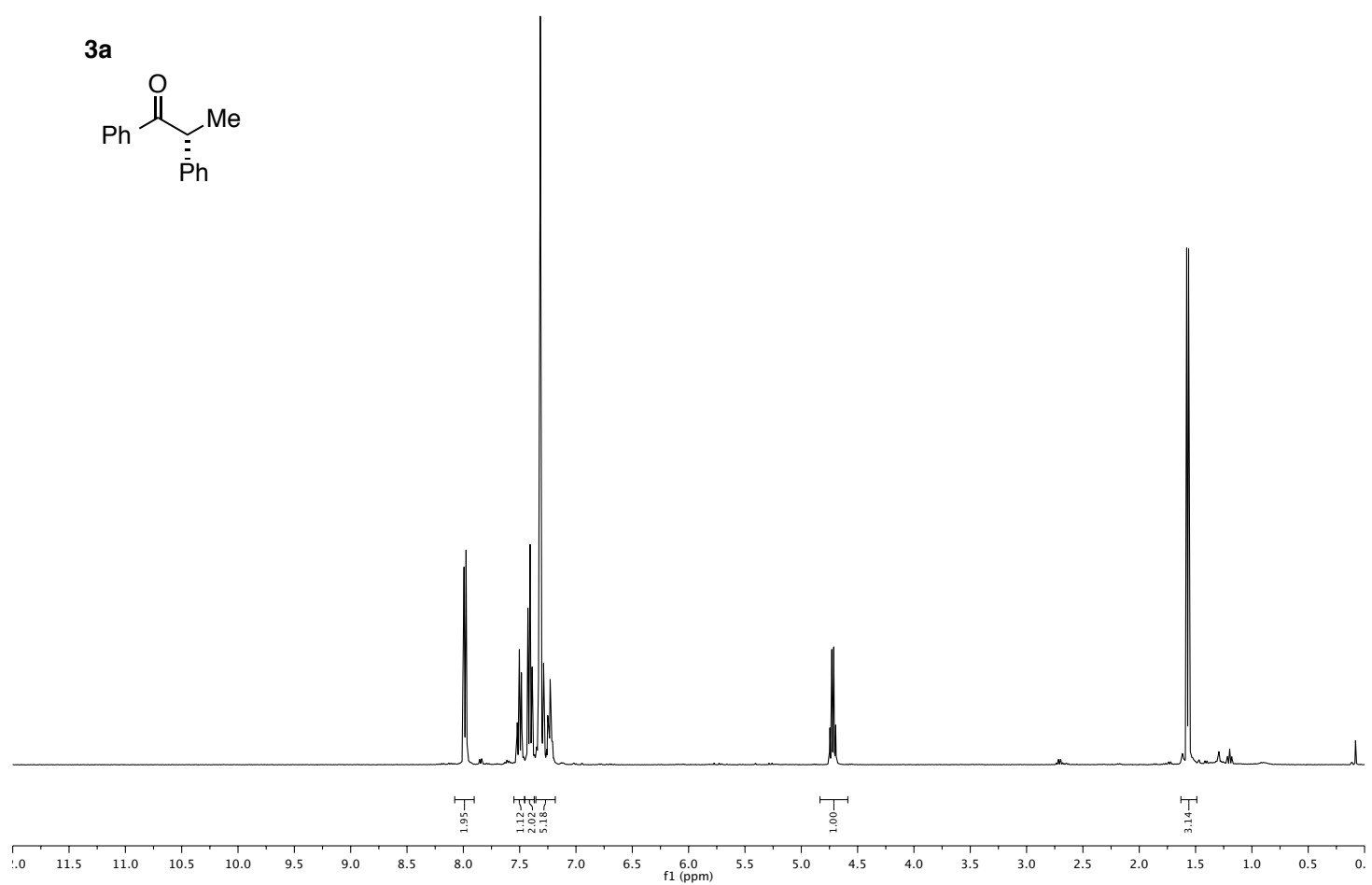
J. Am. Chem. Soc. Supporting Information

S 58

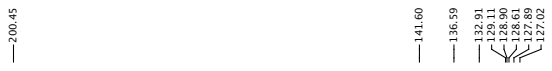

$3 a$<smiles>C[C@H](C(=O)c1ccccc1)c1ccccc1</smiles>

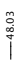
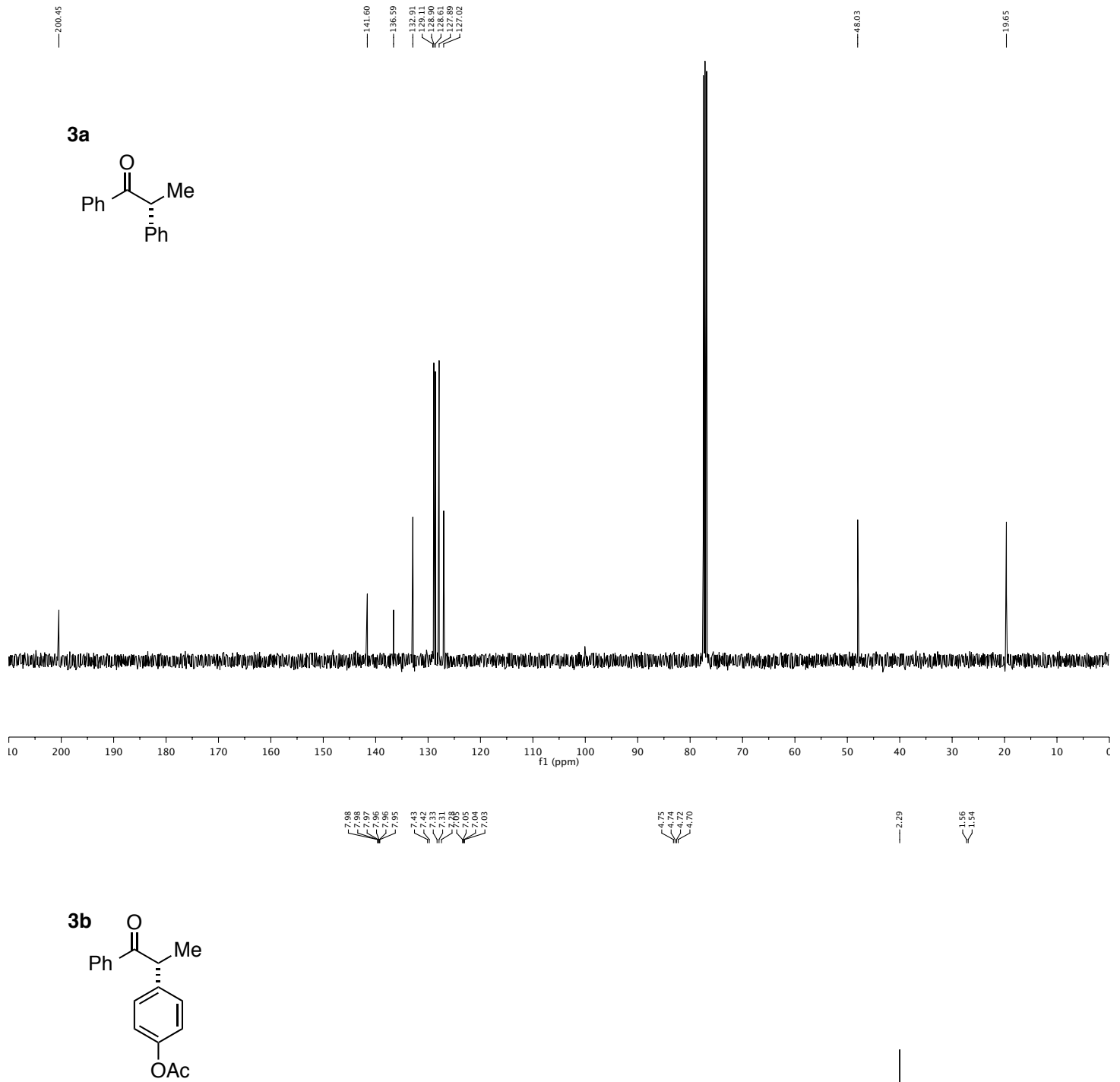

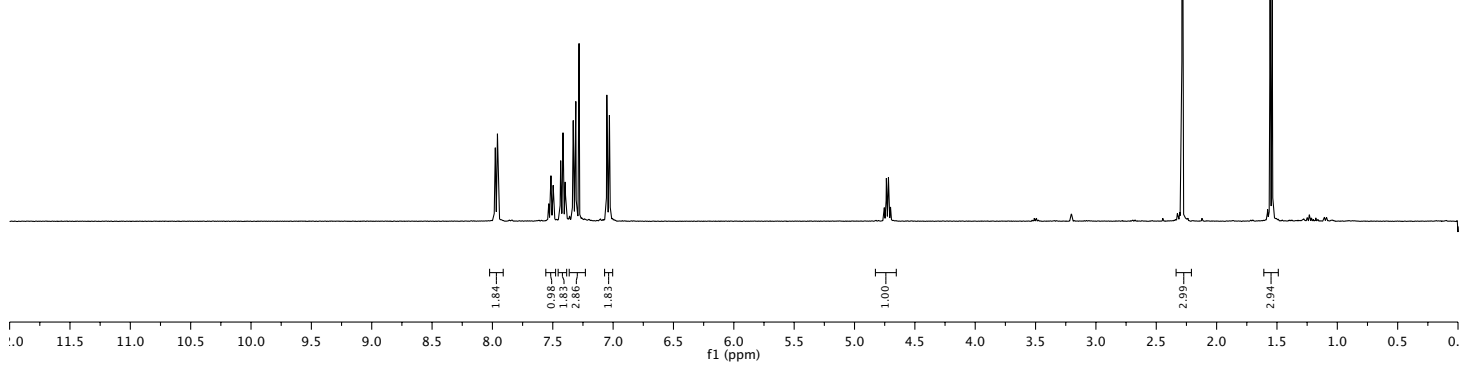




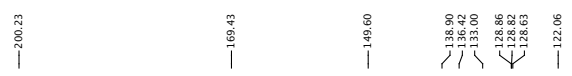

î
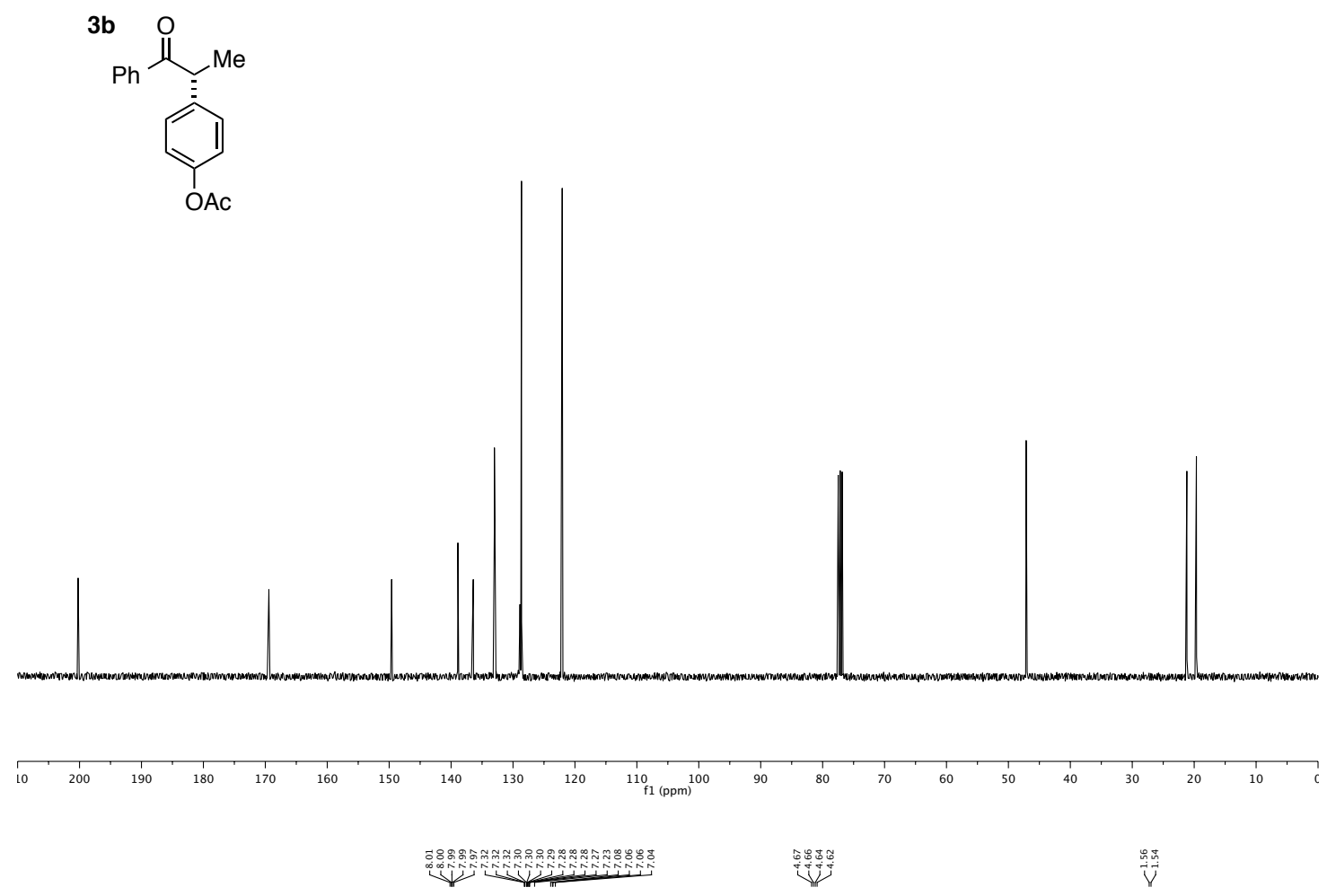

3c<smiles>C[C@H](C(=O)c1ccc(F)cc1)c1ccccc1</smiles>

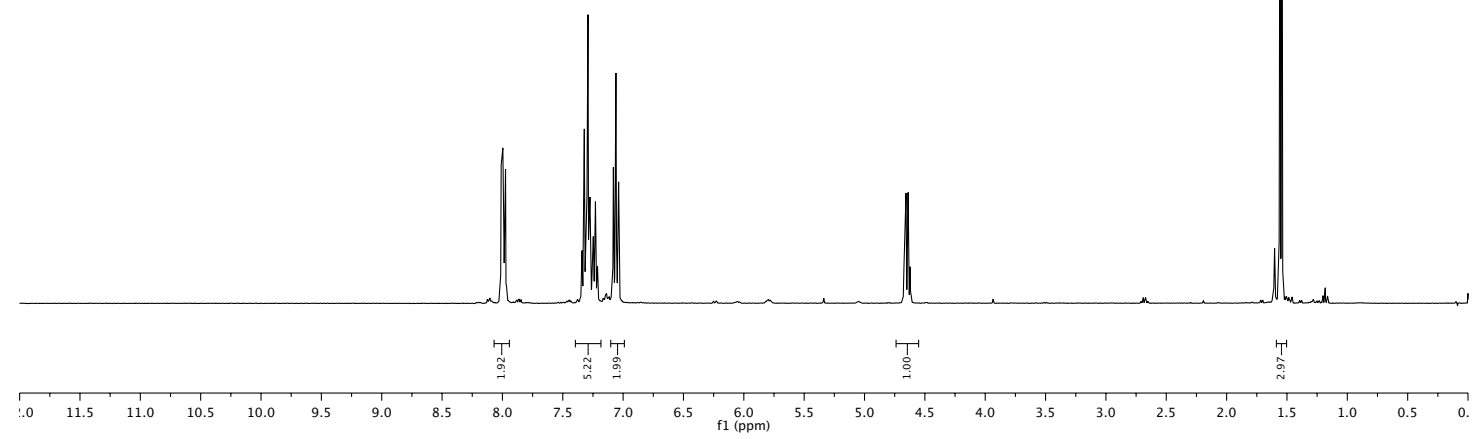



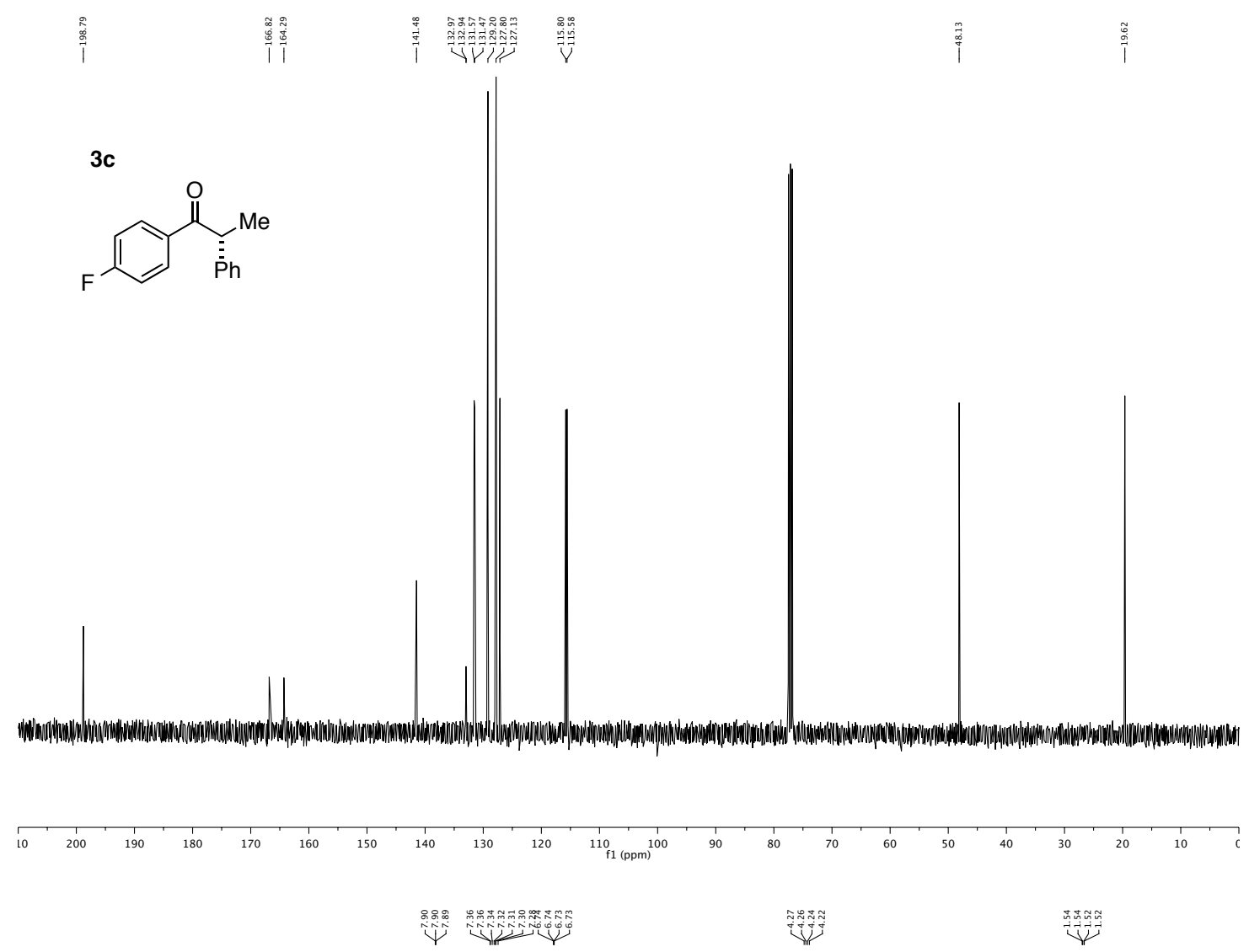

3d<smiles>CC(C(=O)c1ccoc1)c1ccccc1</smiles>

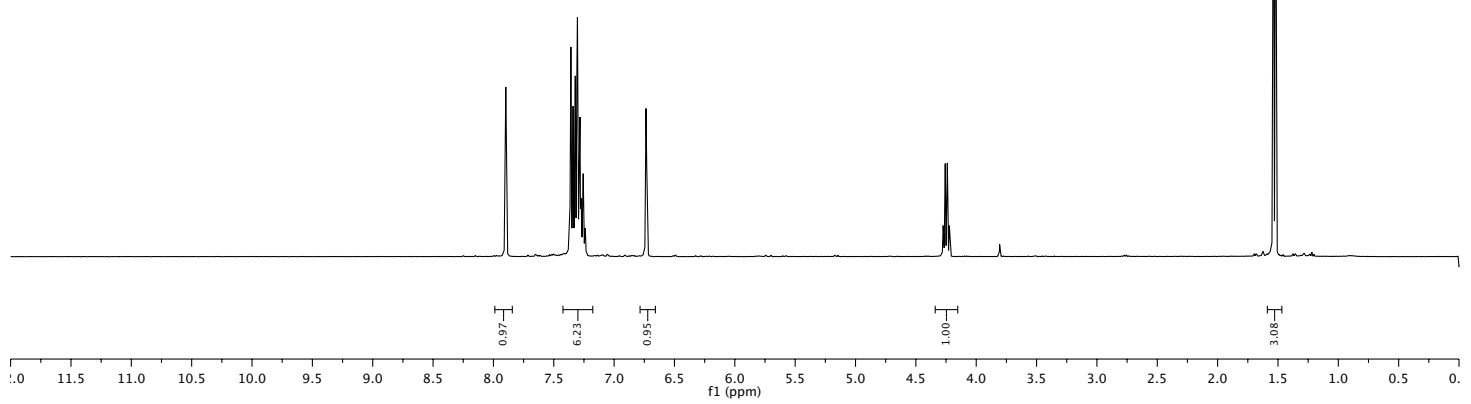



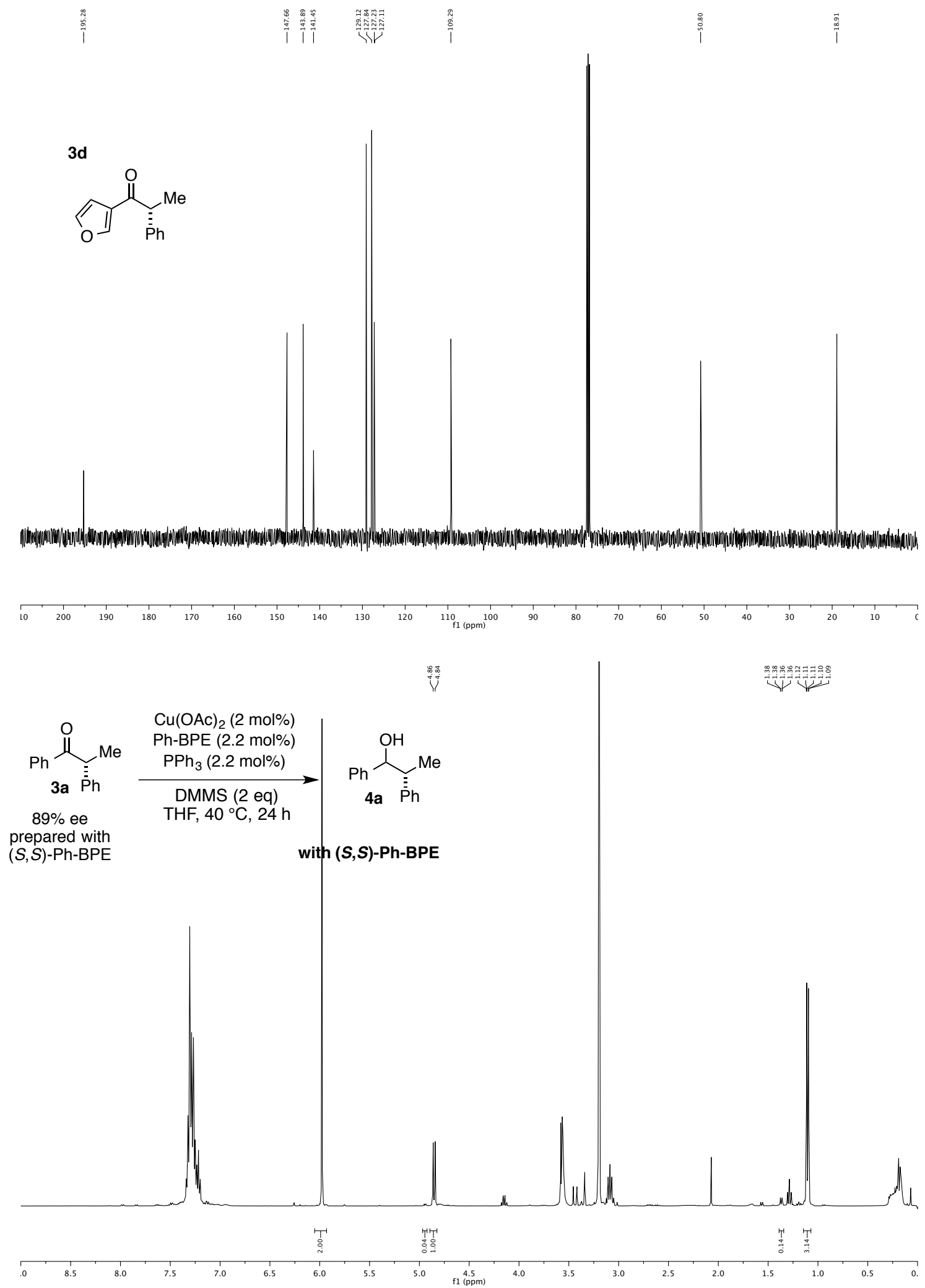


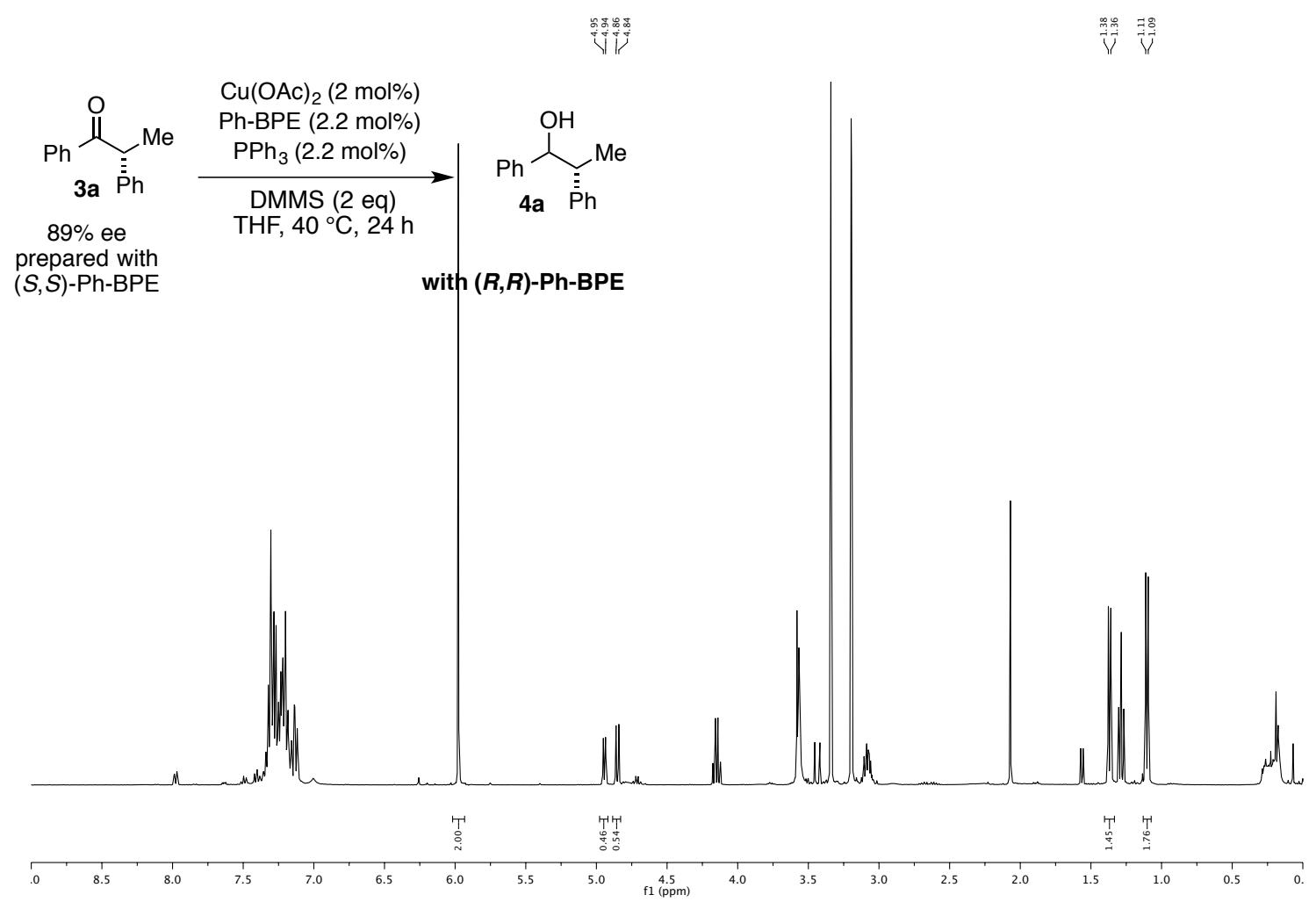

Yบソ<smiles>O=C(OC(=O)c1ccc2occc2c1)c1ccc2occc2c1</smiles>

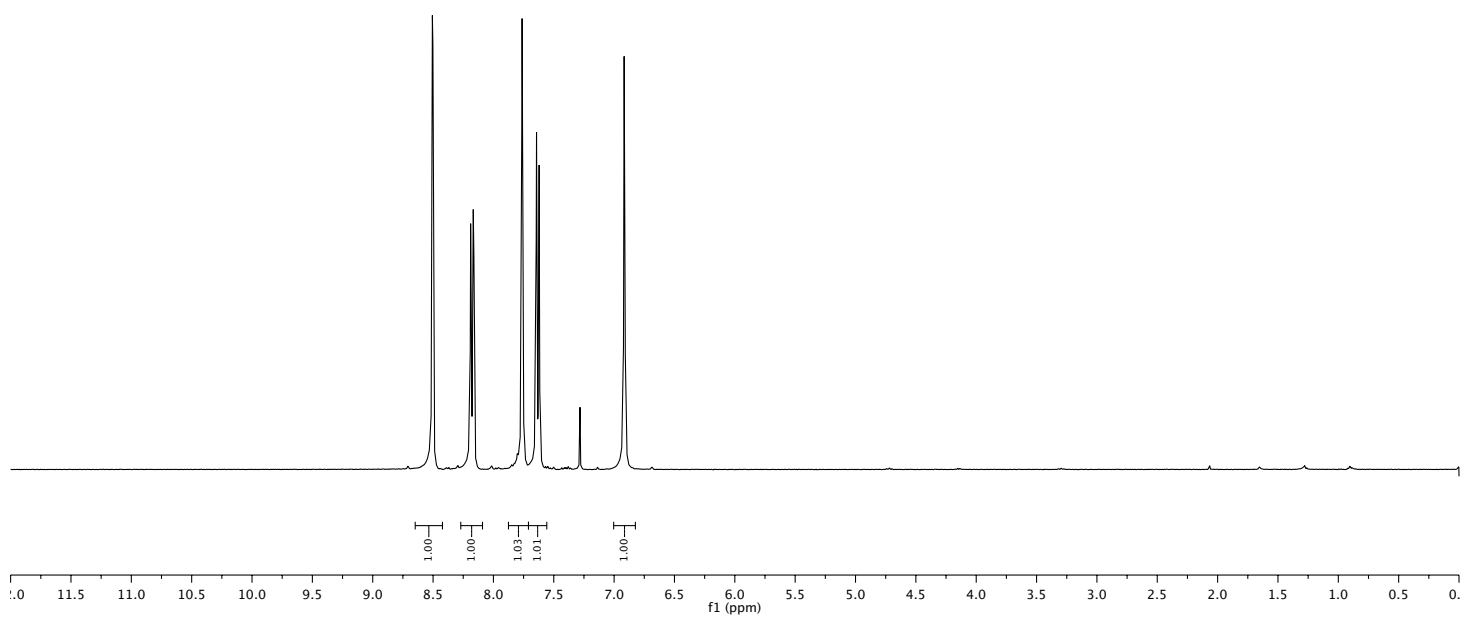




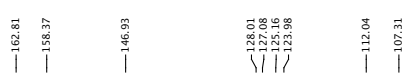<smiles>O=C(OC(=O)c1ccc2occc2c1)c1ccc2occc2c1</smiles>
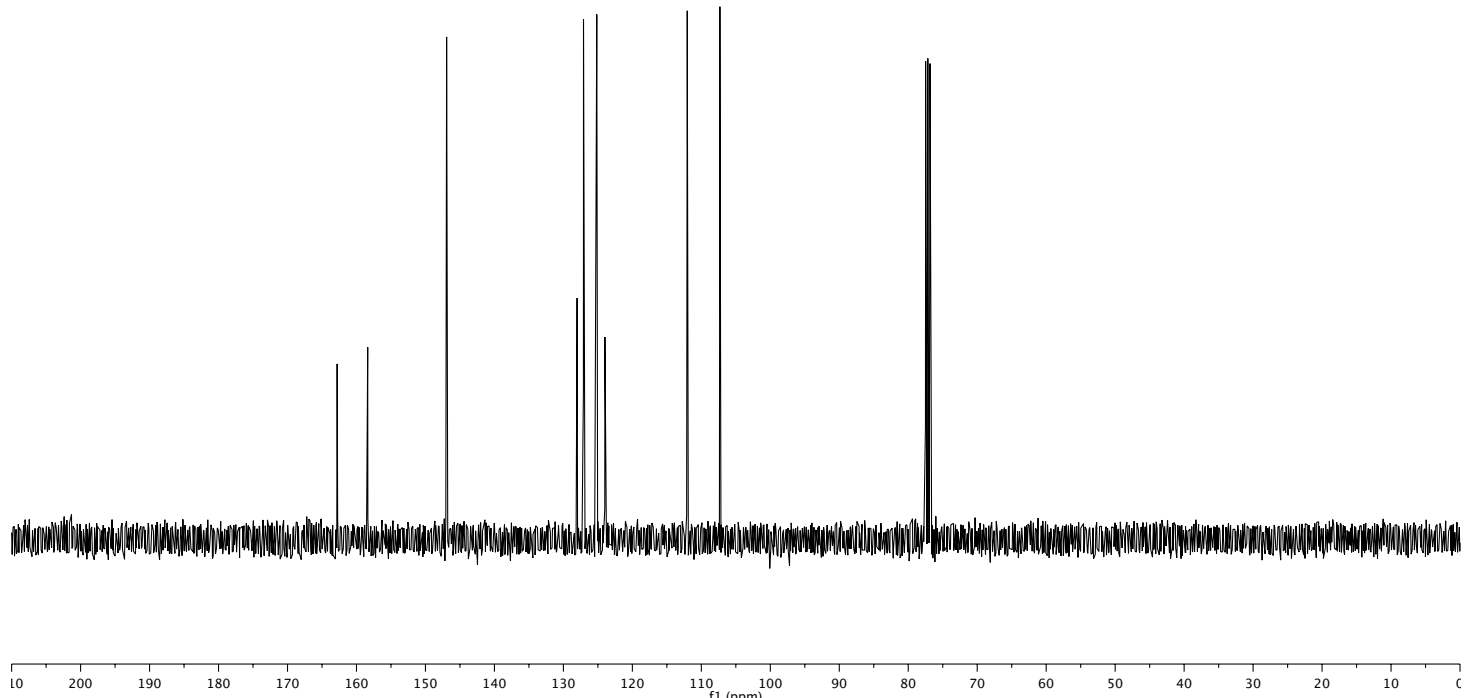

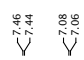

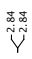<smiles>Cc1sccc1C(=O)OC(=O)c1ccsc1C</smiles>

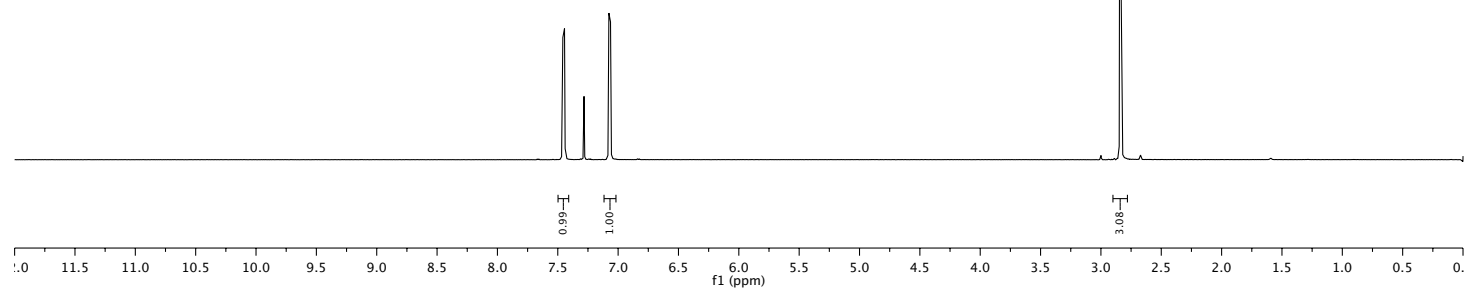



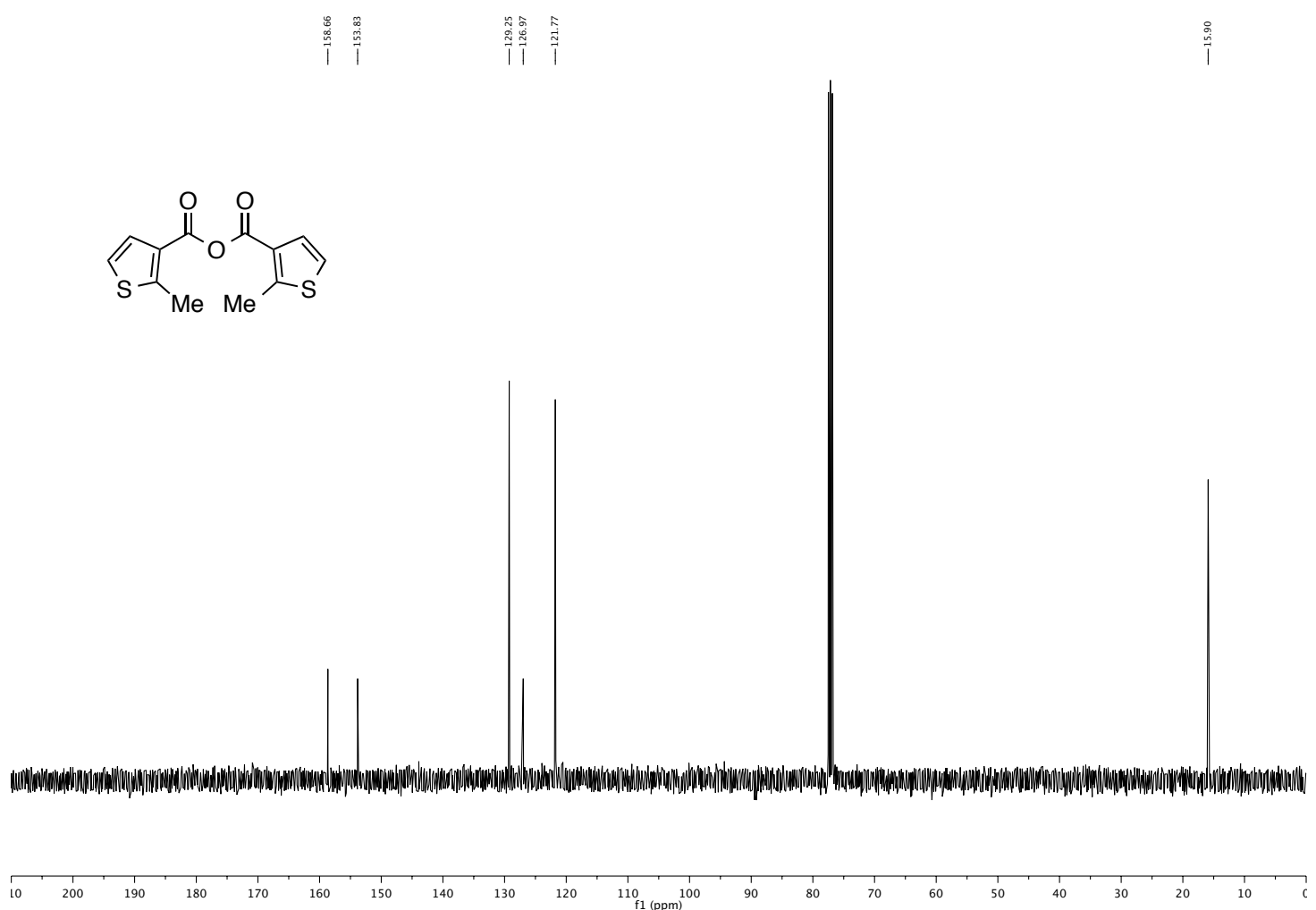

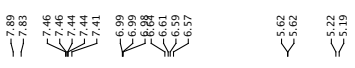
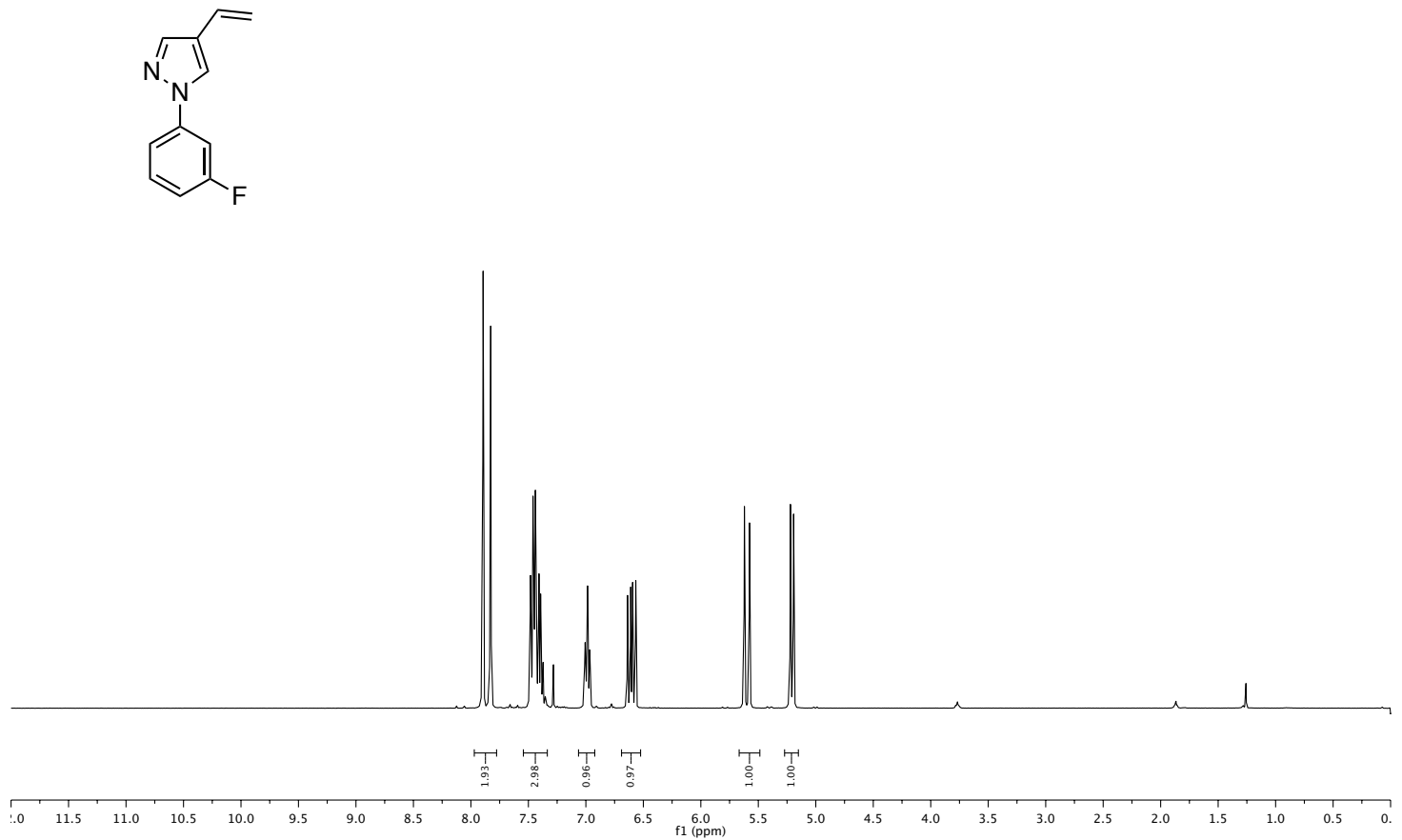


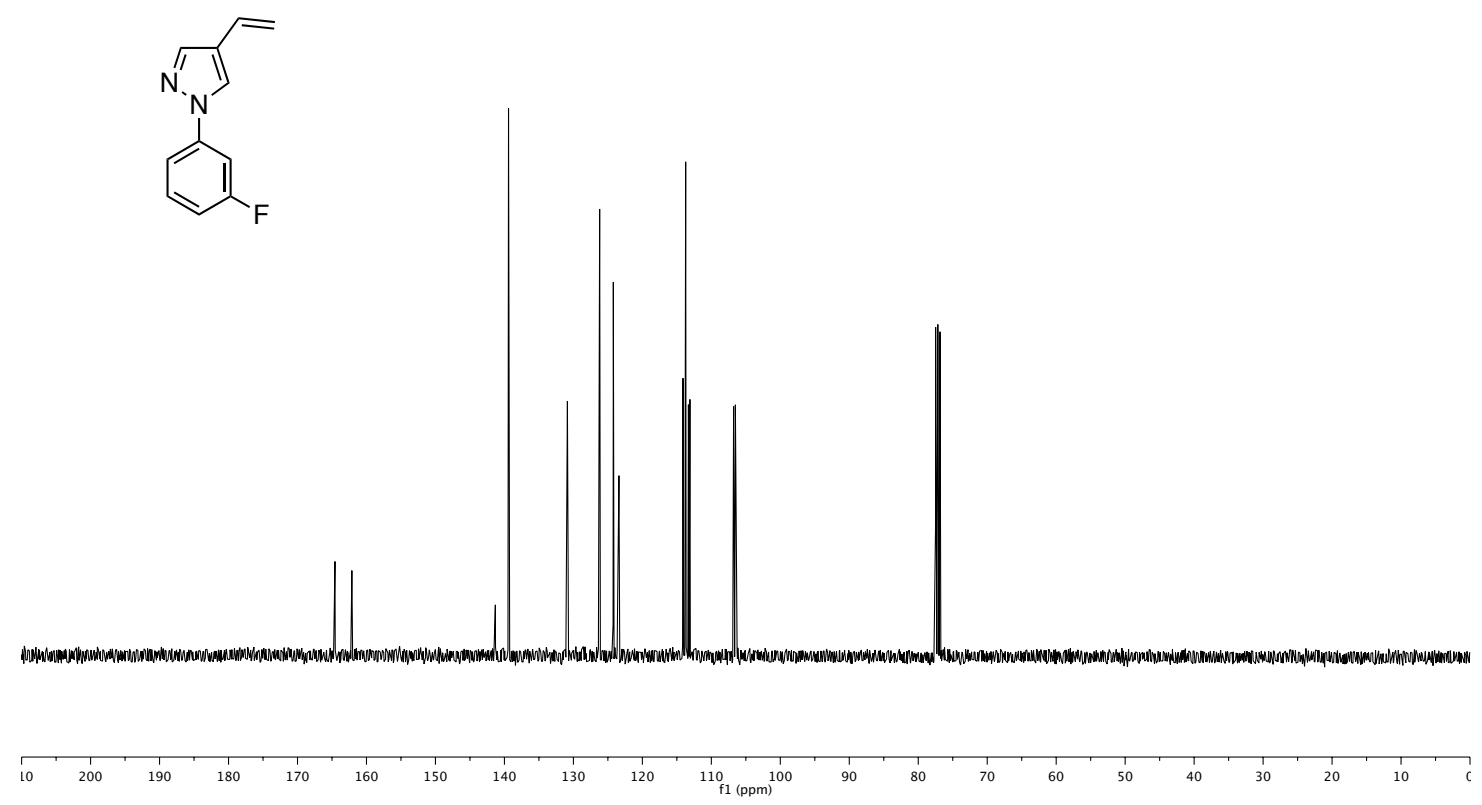

